NBER WORKING PAPER SERIES

\title{
A LONG AND A SHORT LEG MAKE FOR A WOBBLY EQUILIBRIUM
}

\author{
Nicolae B. Gârleanu \\ Stavros Panageas \\ Geoffery X. Zheng \\ Working Paper 28824 \\ http://www.nber.org/papers/w28824 \\ NATIONAL BUREAU OF ECONOMIC RESEARCH \\ 1050 Massachusetts Avenue \\ Cambridge, MA 02138 \\ May 2021, Revised October 2021
}

We would like to thank Itamar Dreschler and seminar participants at the NBER Summer Institute Asset Pricing Program, the UW Foster School of Business, the Shanghai Advanced Institute of Finance, and UNC Kenan-Flagler Business School for their useful comments on the paper. The views expressed herein are those of the authors and do not necessarily reflect the views of the National Bureau of Economic Research.

NBER working papers are circulated for discussion and comment purposes. They have not been peer-reviewed or been subject to the review by the NBER Board of Directors that accompanies official NBER publications.

(C) 2021 by Nicolae B. Gârleanu, Stavros Panageas, and Geoffery X. Zheng. All rights reserved. Short sections of text, not to exceed two paragraphs, may be quoted without explicit permission provided that full credit, including $(\odot$ notice, is given to the source. 
A Long and a Short Leg Make For a Wobbly Equilibrium

Nicolae B. Gârleanu, Stavros Panageas, and Geoffery X. Zheng

NBER Working Paper No. 28824

May 2021, Revised October 2021

JEL No. G11,G12

\section{ABSTRACT}

We document the historically bad performance of shorting strategies in late 2020 and early 2021. Short sellers started retreating several weeks before the dramatic growth in the online discussion of January 2021 and across numerous stocks, the majority of which were not heavily discussed online and did not experience an unusual increase in retail buying volume. We provide a model to explain how fears among short sellers can become self-fulfilling and lead to "run-type" behavior. The model also provides a novel explanation why rational short sellers may choose to leave the market even as mispricing widens.

Nicolae B. Gârleanu

Haas School of Business

F628

University of California, Berkeley

Berkeley, CA 94720

and NBER

garleanu@haas.berkeley.edu

Stavros Panageas

Anderson School of Management

University of California, Los Angeles

110 Westwood Plaza

Los Angeles, CA 90095-1481

and NBER

stavros.panageas@anderson.ucla.edu
Geoffery X. Zheng

New York University

geoff.zheng@nyu.edu

An appendix is available at http://www.nber.org/data-appendix/w28824 
We show that late 2020 to early 2021 was a historically bad period for shorting strategies. Numerous press articles were dedicated to one stock, GameStop, whose price sky-rocketed in January 2021 due to the strong demand by non-professional traders participating in online forums. However, a broad-based decline in short selling had begun almost two months before the dramatic events of January 2021. As early as November 2020, short interest went down in a significant number of stocks that were much larger than GameStop, did not attract the attention of the online crowd, and did not experience any notable change in retail purchase volume.

The timing of the retreat suggests that it was not a direct reaction to GameStop losses $1^{1}$ At the same time, the unremarkable behavior of retail purchase volume for the numerous stocks whose short interest declined suggests that this retreat was not the result of widespread "short squeezes," like the one experienced by GameStop. Rather, we contend that the behavior of short sellers during this period may be better described as a fearful reaction to early warning signs of a shifting market environment.

Motivated by these events, in this paper we attempt to explain why short selling may be a particularly fickle strategy, susceptible to "run-type" behavior. In particular, we provide a novel reason - unrelated to the well-known Shleifer and Vishny (1997) "limits to arbitrage" argument - why rational short sellers may choose to abandon a market even as prices increasingly deviate from fundamentals.

We next provide more detail on our empirical findings, outline the ingredients of the model, and summarize the basic intuitions for our findings.

As has been extensively reported, in January 2021 the WSB subreddit, an online forum where contributors exchange views on trading, saw explosive growth, largely centered around GameStop. Consistent with articles in the popular press linking this online discussion with retail purchases, we show that high-frequency fluctuations of GameStop mentions on the WSB subreddit exhibited a very high correlation (above 90 percent at hourly intervals) with retail purchases of GameStop. This strong high-frequency correlation suggests that the WSB subreddit was an effective vehicle in coordinating retail purchases for this particular stock.

\footnotetext{
${ }^{1}$ In this context, it is worth noting that the dollar value of GameStop shares shorted as of December 31, 2020 was $\$ 1.3$ billion, $0.1 \%$ percent of the total value of all shares shorted in the US stock market at that time.
} 
Remarkably, many other high-short-interest stocks experienced significant declines in short interest starting in November 2020 (thus, six to eight weeks before the GameStop events), even though these stocks were not heavily discussed on the WSB subreddit, did not experience any unusual increase in retail purchases, and were much larger than GameStop. Concurrently, the strategy of shorting these stocks performs strikingly poorly ${ }^{2}$ For such stocks there appears to be no evidence of a coordinated short squeeze by the WSB subreddit participants, yet short interest nonetheless declined. Interestingly, this decline appears to be permanent, suggesting a shift in equilibrium.

Using these events as backdrop, we develop a model that explains why short selling can be unstable. We are not interested in explaining the behavior of GameStop, but rather situations in which a short-seller exit is not the result of a short squeeze. In particular, the model emphasizes the feedback loop between the Sharpe ratio and short interest and shows that the same fundamentals can lead to equilibria in which short interest is high, intermediate, or zero. This equilibrium multiplicity is a time-honored device to illustrate the possibility of a run: a shared perception of a shift in equilibrium is enough to cause an abrupt short-seller exit. In addition, the model provides a novel explanation for why rational short sellers may choose to abandon a market even as mispricing widens.

The model features investors with heterogeneous beliefs about the expected return of a positive-supply, risky stock: one group is optimistic, while the other holds rational beliefs ${ }^{3}$ This difference of opinion between investors prompts them to trade with each other, with the rational investors having an incentive to short the stock whenever the expected excess return becomes negative. Shorting stock requires borrowing it, for a fee determined endogenously in the lending market as a result of bargaining.

As is recognized, the presence of lending fees modifies the returns experienced by both long and short investors. The equilibrium risk compensation (the "Sharpe ratio") is impacted both by the magnitude of the lending fee and the fraction of a representative lender's shares

\footnotetext{
${ }^{2}$ An illustration of the gloomy mood among short sellers at that time was the emblematic decision of Citron Research to stop publishing short selling research after over 20 years: "Citron to stop publishing short-selling research, Andrew Left says" (https://financialpost.com/investing/ citron-research-andrew-left-stop-short-selling-research-publishing).

${ }^{3}$ Motivated by the empirical fact that stocks with high short interest tend to have low subsequent returns, we assume that the comparatively more pessimistic investors are actually rational, but this is not an essential assumption for our results.
} 
that are shorted. (We refer to this quantity as short interest.4) All else equal, a higher short interest acts as an increased subsidy for long positions. This basic property of the model is responsible for equilibrium multiplicity. To see this, consider two equilibria. In equilibrium A the (instantaneous) Sharpe ratio is higher than in equilibrium B, while short interest is lower. In equilibrium B, the relatively low Sharpe ratio entices short sellers to pay the lending fee, which in turn provides lending income to the long investors and makes them content to hold long positions, despite the low Sharpe ratio. By an analogous argument, in equilibrium A the relatively higher Sharpe ratio deters short sellers, who find the post-lending fee Sharpe ratio to be unattractive; at the same time, the relatively high Sharpe ratio in equilibrium $\mathrm{A}$ makes long investors content to hold a long stock position despite the lack of lending income.

The discussion in the above paragraph takes the wealth share of short sellers at a given point in time as fixed. One advantage of our dynamic setup is that we can study the evolution of the wealth shares depending on whether investors coordinate on a high or a low shorting equilibrium. We show that the wealth growth of short sellers is higher in the high shorting equilibrium ( $\mathrm{A}$ in the above paragraph) than in the no shorting equilibrium (B). An implication is that the (stochastic) steady-state fraction of wealth controlled by short sellers is lower in the equilibrium without shorting. Since the Sharpe ratio is increasing in the wealth share of these investors, the steady state Sharpe ratio may well be lower if investors coordinate on the low-shorting equilibrium than if they coordinate on the high-shorting equilibrium.

To fully explore the implications of this dynamic effect in a more realistic setup, we extend the model to allow for multiple stocks with endogenous participation. After showing that our conclusions from the single-stock economy extend to the multi-stock economy, we focus on the case where there is a large and a small stock, with disagreement affecting only the small stock $5^{5}$ For realism, we assume that only a small fraction of investors pay attention to the small stock and incur a small participation cost in doing so. In that extended

\footnotetext{
${ }^{4}$ Short interest commonly refers to the number of shares shorted as a fraction of the float. This is a monotonic transformation of the quantity to which we refer as short interest.

${ }^{5}$ With this assumption, the interest rate becomes essentially fixed and therefore fluctuations in the Sharpe ratio are mirrored in the price-dividend ratio of the small stock. By contrast, in the baseline model the assumption of log utility and i.i.d. dividend growth imply that fluctuations in the Sharpe ratio are exactly offset by fluctuations in the interest rate, leaving the price-dividend ratio unaffected.
} 
version of the model, we show that the shift to a low shorting equilibrium causes rational investors to exit the market for the small stock, since remaining in a market without a trading opportunity is not worth paying that participation cost. The exit of rational shortsellers causes a simultaneous rise in the price of the stock - consistent with the empirical observation that bad returns of shorting strategies coincide with drops in short interest. $]^{6}$

The paper is organized as follows: After a brief literature review, we motivate the model with some empirical observations (Section 1). The baseline model is contained in Section 2 . Section 3 contains the main analytical results. Section 4 discusses dynamic properties of the equilibria and some empirical implications of the model. Section 5 presents the extensions to multiple stocks and Section 6 concludes. Proofs, detailed descriptions of the data, and additional results are contained in the appendix.

\section{Related Literature}

Our work relates to several strands of the asset-pricing literature. The most closely related one considers the joint determination of lending fees, short interest, and returns. In particular, D'Avolio (2002), Duffie et al. (2002), Vayanos and Weill (2008), Banerjee and Graveline (2013), and Atmaz et al. (2020) consider explicit frictions to lending and borrowing shares, which translate into non-zero lending fees that in turn impact expected returns.7 Similar to D'Avolio (2002) $]^{8}$ Banerjee and Graveline (2013), and Atmaz et al. (2020), the lending and spot markets clear simultaneously in our paper, but we use a different micro-foundation to obtain a positive lending fee. Specifically, we don't impose any hard constraint on the shares that a long investor can lend. Instead, we obtain a positive lending fee by assuming that the process of matching share lenders and borrowers is a time-consuming activity, which requires compensation, similar in spirit to Duffie et al. (2002). By taking that route, the model allows for a more general specification of the "supply curve" of lendable shares; it is not confined

\footnotetext{
${ }^{6}$ This was the case, for example, in January 2021.

${ }^{7}$ Such frictions also motivated the empirical studies of Geczy et al. (2002), Lamont (2012), Jones and Lamont (2002), Kaplan et al. (2013), Porras Prado et al. (2016), and Asquith et al. (2005) among others.

${ }^{8}$ More precisely, to a working-paper version of this study, which contains a theoretical model that did not appear in the published article.
} 
to vertical supply curves 9 This specification of the supply curve for lendable shares leads to a feedback loop between the Sharpe ratio and short interest that is not present in the aforementioned papers (which feature unique equilibria). In addition, our model allows us to explore the dynamic effects of an equilibrium shift, driven by the endogenous fluctuations in the wealth shares of the different agents ${ }^{10}$

An even larger number of papers assume that shorting is prohibited and analyze implications for returns. Indicative papers here include Harrison and Kreps (1978), Miller (1977), Diamond and Verrecchia (1987), Detemple and Murthy (1997), Hong and Stein (2003), and Scheinkman and Xiong (2003). As in Harrison and Kreps (1978) and Miller (1977), we model the motive for trade in our paper in the convenient form of (dogmatic) differences of opinions among agents.

A large body of work studies the empirical relation between short interest and stock returns. Seneca (1967), Senchack and Starks (1993), Desai et al. (2002), Diether et al. (2009), Asquith et al. (2005), Blocher et al. (2013), Beneish et al. (2015), and Dechow et al. (2001) study the cross-sectional relation and find that stocks with higher short interest underperform those with lower short interest. Later work by Cohen et al. (2007) and Boehmer et al. (2008) uses proprietary data on quantities lent as well as shorting fees and finds consistent results. Duong et al. (2017) studies the empirical relation between lending fees and stock returns and finds that high lending fees predict lower future returns. Drechsler and Drechsler (2014) documents that asset pricing anomalies concentrate in stocks with high shorting fees. Lamont and Stein (2004) studies the information content in aggregate short interest and finds that short interest declined as stock market valuations rose in the late 90's. Rapach et al. (2016) shows that the predictive power of aggregate short interest stems predominantly from a cash-flow channel. Kaplan et al. (2013) finds no evidence of a change in expected stock returns in response to an exogenous change in the supply of lendable shares.

Our paper is consistent with the main findings of the empirical literature, such as a

\footnotetext{
${ }^{9}$ An exception is Atmaz et al. (2020). In their model, individual agents' supply curves are vertical, but the aggregate supply curve has finite elasticity due to composition effects when aggregating across agents.

${ }^{10}$ The fact that shorting requires borrowing shares and is subject to natural collateral requirements has several interesting general equilibrium implications, as explored by Fostel and Geanakoplos (2008), Simsek (2013), and Biais et al. (2021). In contrast, our model focuses on the general equilibrium implications of the associated lending fees.
} 
declining Sharpe ratio with respect to short interest and lending fees and short sellers earning a risk compensation even after controlling for the lending fee. In addition, the model can help address the intriguing findings of Kaplan et al. (2013), as we show in Section 4.3.

Our paper also relates to a sizable theoretical literature analyzing multiple equilibria in asset pricing and macroeconomics. Multiple equilibria can arise through a number of mechanisms, chief among them a) bubbles (or money) in OLG economies, b) increasing returns to scale and production externalities, and c) portfolio constraints ${ }^{11}$ To our knowledge, ours is the first paper in which multiple equilibria are due to shorting fees that can make a long position sufficiently attractive to sustain a higher level of the short interest.

Finally, several recent papers target specifically the set of events involving GameStop. See, for instance, Pedersen (2021) and Allen et al. (2021).

\section{Empirical Motivation}

We motivate our theoretical model with several empirical facts. The online-forum discussion of January 2021 focused narrowly on GameStop (GME) and only a few other stocks, while a large number of highly shorted stocks experienced significant price increases relative to the market concurrently with a declining short interest. The broad retreat of the short sellers (across a large number of highly shorted stocks) started about two months before the GameStop events. In addition, unlike GameStop, there is no evidence of a broad short squeeze affecting these stocks, and the timing of events is not consistent with a "balance-sheet-style" contagion whereby short sellers react to the losses they experienced in GameStop ${ }^{12}$ Thus, a couple of obvious candidate explanations for this broad decline in short interest appear incorrect, suggesting the possibility that the behavior of short sellers was the result of fear, a fear strong enough to keep them away from the market even as the prices of the stocks that they believed to be mispriced as of November 2020 climbed even higher.

\footnotetext{
${ }^{11}$ We refer the reader to the survey by Benhabib and Farmer (1999), which lists and discusses the different mechanisms that lead to multiple equilibria and indeterminacies. Recent examples of papers using multipleequilibrium models in asset pricing include Gârleanu and Panageas (2021), Khorrami and Zentefis (2020), Khorrami and Mendo (2021), Zentefis (2018), and Farmer and Bouchaud (2020).

${ }^{12}$ See, e.g., Kyle and Xiong (2001).
} 


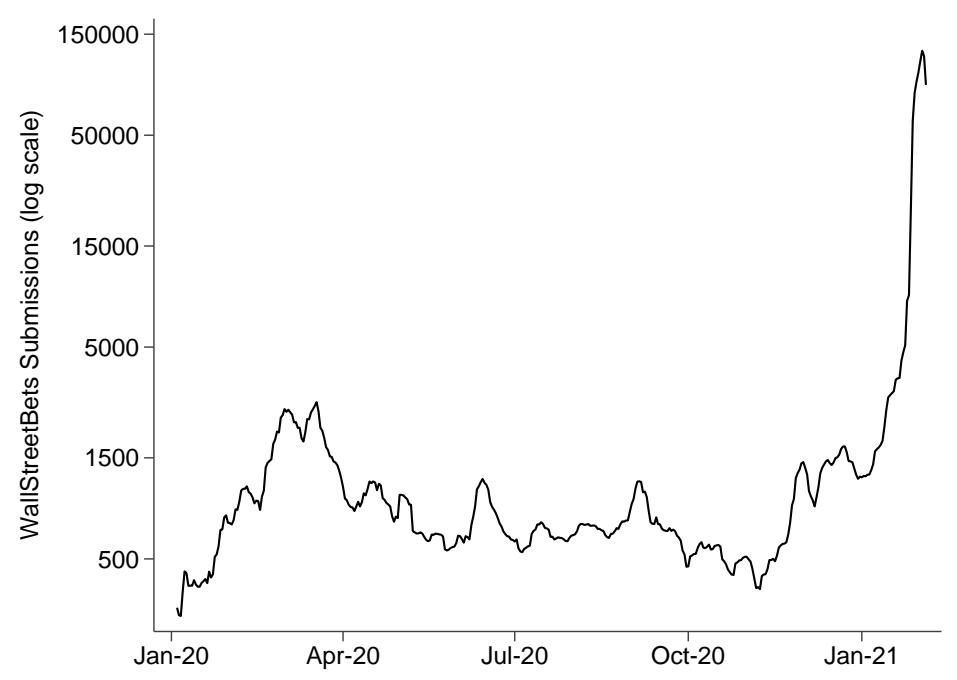

Figure 1: Seven-day moving average of daily submissions to the WallstreetBets subreddit (January 1, 2020 - February 7, 2021). The vertical axis is on a logarithmic scale.

In terms of data, we combine standard academic data-sets with social media posts collected from the WallstreetBets subreddit (WSB), a subdomain of the Reddit website. (For a detailed description of the data collection process, see Appendix F.) Reddit is a large online website featuring specialized communities in which users post messages and other users can comment on these posts in message-board fashion. Users on the WSB subreddit actively discuss financial news, investments, and individual securities with one another. We plot the daily submissions to WSB in Figure 1 on a logarithmic scale. Although the subreddit has existed for a number of years (it was created in 2012), daily activity on WSB grew exponentially in early January 2021.

It is important to note that the vast majority of the discussion on the WSB subreddit focused on a very limited number of stocks. Our textual analysis enables the creation of highfrequency time series of ticker-mentions by aggregating mentions within a time interval. As can be seen in Figure 15 in Appendix G, the "lion's share" of mentions centered around GameStop. From December 1, 2020 to February 1, 2021, six most-discussed stocks account for about half of all discussion. That proportion peaks to over $80 \%$ in the week of January 18-25, primarily driven by the rise in GameStop mentions.

The popular press attributed the stratospheric returns of GameStop during that period to the active discussion of that stock on the WSB subreddit. One advantage of using high- 


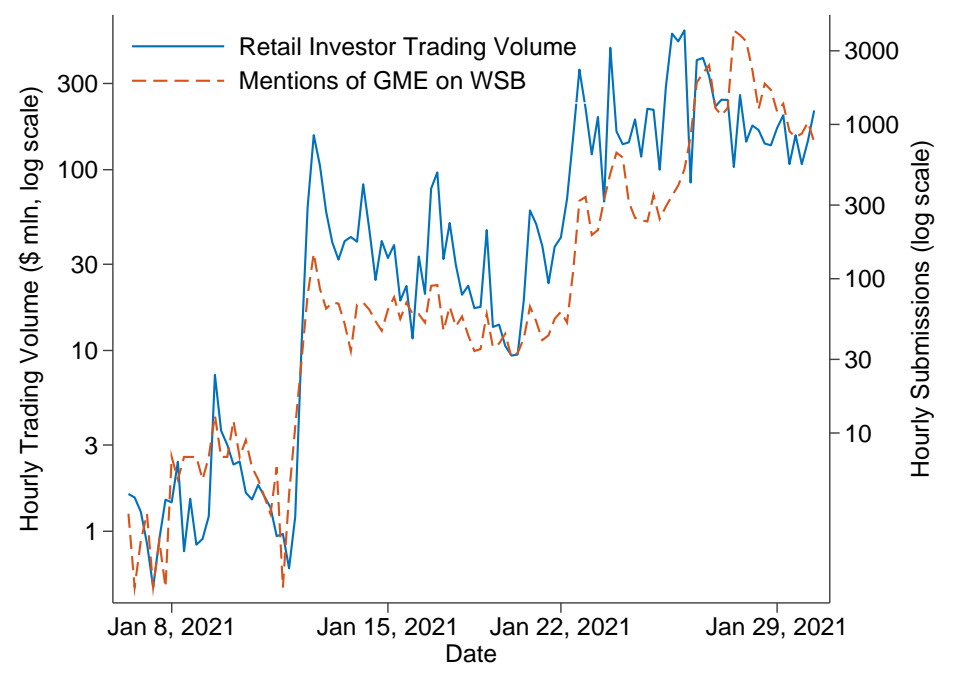

Figure 2: Retail trading volume in GME (January 7 - January 29, 2021). Hourly trading volume in GME, measured using the methodology of Boehmer et al. (2020), plotted together with hourly mentions of the GME ticker on the WallStreetBets subreddit. Both vertical axes are on logarithmic scales.

frequency data on WSB mentions is that we are able to identify a strong "real-time" link between mentions of GameStop on WSB and retail trading volume, which we measure in TAQ data with the methodology of Boehmer et al. (2020). Figure 2 plots the two series at hourly frequency. Over the three trading weeks shown, GameStop mentions on WSB and retail trading purchases exhibit strikingly strong comovement (0.93 rank correlation).

While the events surrounding GameStop made the front page of many newspapers, the press paid less attention to the broader trend that preceded the events surrounding GameStop by about two months. Specifically, as early as mid-November 2020, the returns of shorting strategies started to collapse across a wide array of stocks. The remainder of this section documents that a) the returns of shorting strategies between November 2020 and January 2021 were historically bad and the losses on short positions extended to a wide array of highly shorted stocks, not just the few stocks discussed on WSB, b) short interest declines significantly both in November 2020 and in January 2021, and c) unlike GameStop, which saw a high increase in its retail purchase volume, most other highly shorted stocks saw a decline in short interest but without an unusual behavior of retail purchase volume.

To illustrate point a), in Figure 3 we plot the cumulative returns to an equal-weighted 
portfolio that "bets against the shorts." The portfolio is long the top decile of Russell 3000 stocks, ranked by short interest, and short the broad market. Stock return data are from CRSP and short interest data are from the SEC.13 The figure shows that the bettingagainst-the-shorts strategy is not particularly profitable (or unprofitable) from late June to mid-November 2020, but becomes strikingly profitable over the following three months. To put this evidence in historical perspective, in Figure 4 we plot a histogram of the monthly returns of this betting-against-the-shorts strategy for as long as data are readily available (since 1973). Figure 4 shows that the January 2021 and November 2020 returns are the highest and second-highest (respectively) in the historical sample. (December 2020 is also in the top decile of the historically observed returns). Figure 16 in Appendix $G$ further shows that November 2020 and January 2021 remain outliers if we exclude the popular reddit tickers in the sample (top left panel), if we only include S\&P500 constituents (i.e., larger stocks) in the formation of the long leg of the portfolio (top right panel), and if we value-weight rather than equal-weight returns while including or excluding popular reddit-tickers (bottom left and bottom right panels, respectively) ${ }^{14}$ These results are hardly surprising, since the returns shown in Figures 4 and 16 correspond to portfolios of which GameStop and the other stocks heavily discussed on the WSB subreddit comprise only a small fraction. Table 1 in Appendix $\mathrm{G}$ presents the results of formal statistical tests (also controlling for Fama-French factors) of whether the returns in November 2020, December 2020, and January 2021 are statistically different from the average return on the betting-against-the-shorts strategy over the full 48-year sample.

Turning to point b), Figure 5 plots a binned scatterplot of short interest on January 15, 2021 against the subsequent realized decline in short interest, in blue. The decline in short interest is concentrated among those stocks with high short interest on January 15, 2021. Figure 5 also shows that the decline in short interest among stocks with high short interest is not just a manifestation of mean reversion. The change in short interest predicted by an $\mathrm{AR}(1)$ model, shown in red in Figure 5, is much smaller than the one observed in the data. ${ }^{15}$

\footnotetext{
${ }^{13}$ For additional details on the construction of this portfolio, see Appendix $\mathrm{F}$.

${ }^{14}$ The popular stocks on the WSB subreddit in January 2021 were: AMC, BBBY, GME, SPCE, TLRY, and TSLA.

${ }^{15}$ Specifically, the predicted changes in short interest come from a regression of short interest of stock $i$ at time $t+1$ against short interest of stock $i$ at time $t$. To account for non-linearities in mean reversion, we
} 


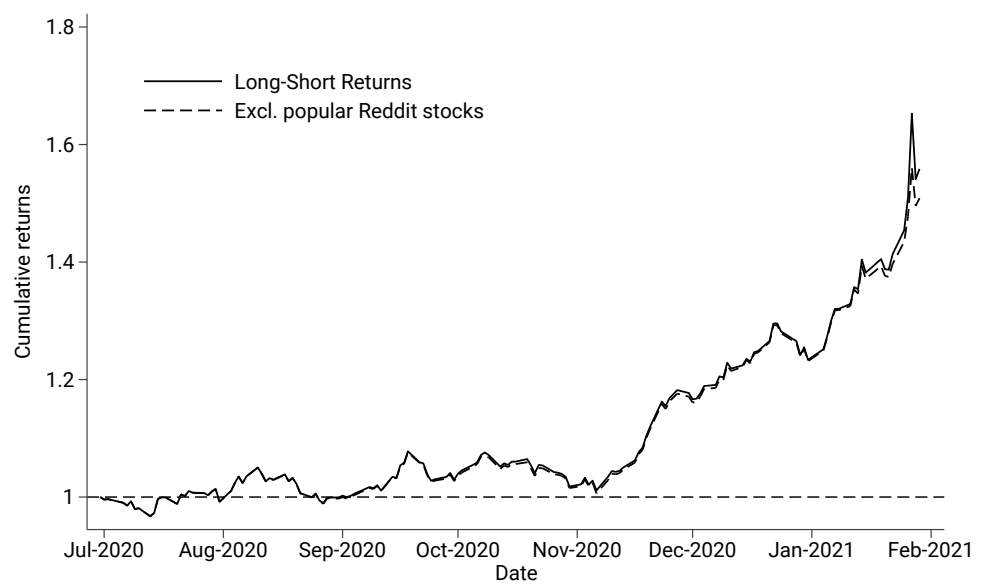

Figure 3: Cumulative Returns (July 2020 - February 2021). Cumulative returns on a strategy long the top decile of stocks by short interest and short the broad market. The returns to an equal-weighted long-short portfolio are shown by the solid black line. The returns to the same portfolio, but excluding the six most-discussed tickers on the WallstreetBets subreddit (AMC, BBBY, GME, SPCE, TLRY, and TSLA), are shown in the dashed black line.

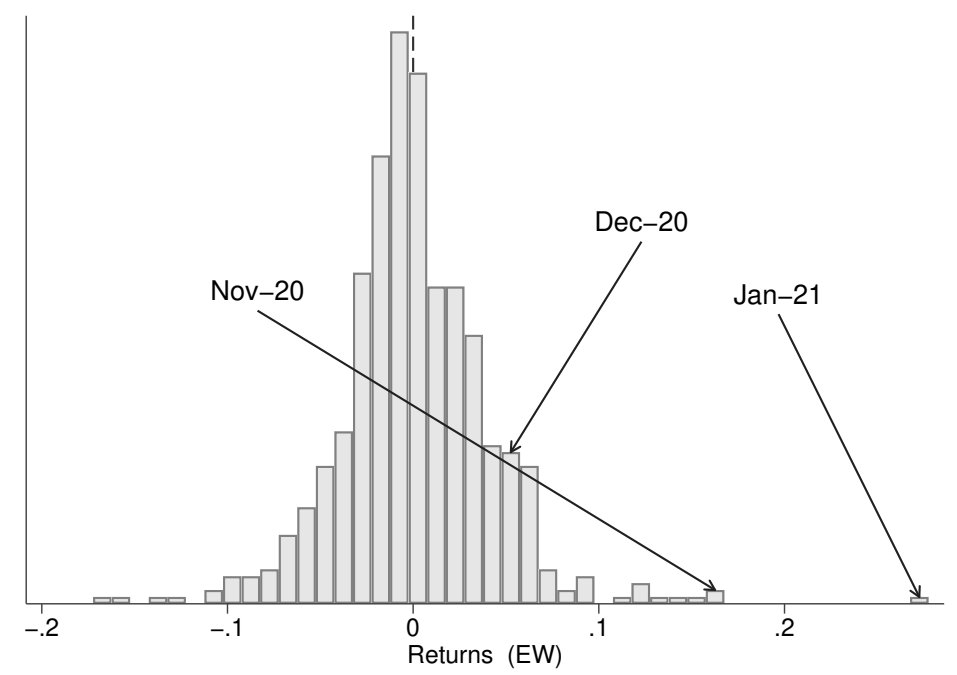

Figure 4: Histogram of monthly returns (1973-2021). Equal weighted returns on a portfolio long stocks in the top decile of short interest and short the market index. The arrows indicate the portfolio returns in the months of November and December 2020 and January 2021.

Figure 17 in Appendix $\mathrm{G}$ repeats the exercise of Figure 5 for the months leading up to the dramatic events of January 2021. The figure suggests that the onset of the decline in short interest begins in mid-November 2020, which is also the time when the returns of shorting also include dummies for the decile of short interest that stock $i$ belongs at time $t$, along with an interaction term between short interest of stock $i$ at time $t$ and the decile dummy. 


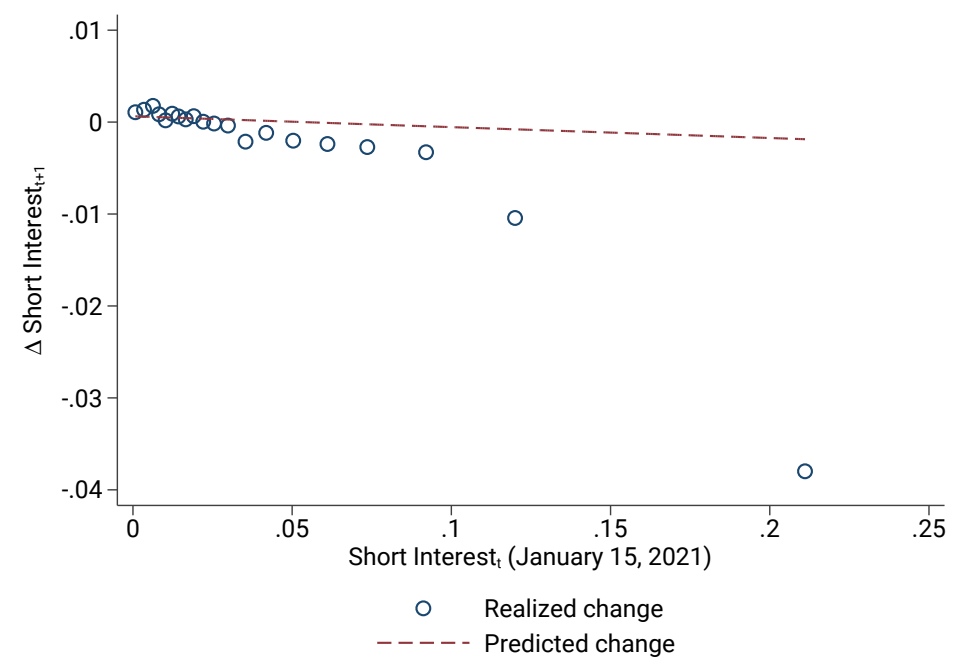

Figure 5: Change in short interest (January 15-January 29, 2021). Stocks are binned into one of twenty groups based on short interest as of January 15, 2021. The average percentage point change in short interest from January 15 to January 29, 2021 for each bin is plotted on the y-axis. Predicted change is computed based on an $\operatorname{AR}(1)$ for each decile of short interest, fitted on historical shorting data.

strategies start exhibiting their historically bad performance in Figure $3{ }^{16}$

To illustrate point c), Figure 6 shows that the broad reduction in short interest in January 2021 cannot be attributed to a similarly broad increase in retail traders' stock purchases. In other words, the numerous stocks that saw large reductions in their short interest did not experience a noticeable change in retail purchase volume. In Figure 6, we plot changes in retail purchase volume against the change in short interest for all stocks in the top decile of short interest as of January 15, 2021. Changes in both retail purchase volume and short interest are reported as standardized $z$-scores using TAQ and SEC data from January 2015 through January 2021. We can see that, while there is a negative slope coefficient, it is economically small and statistically indistinguishable from zero, suggesting no relation between changes in short interest and changes in retail purchase volume. Indeed, the $\mathrm{x}$-axis values of most observations are between -2 and 2, indicating that January 2021 was not an unusual month for retail purchase volume for these highly shorted stocks. This is in sharp contrast

\footnotetext{
${ }^{16}$ Interestingly, December 2020, which was a bad month (bottom $10 \%$ percentile in the historical sample) for shorting returns, but not as bad as November 2020 or January 2021, exhibits smaller declines in the short interest of the portfolio of most shorted stocks (right-most point in the graph). Indeed, the gap between the right-most circle and the dotted AR(1) line in the November 30 and December 15 panels (corresponding to the full month of December 2020) is the smallest among the six plots starting with November 13.
} 


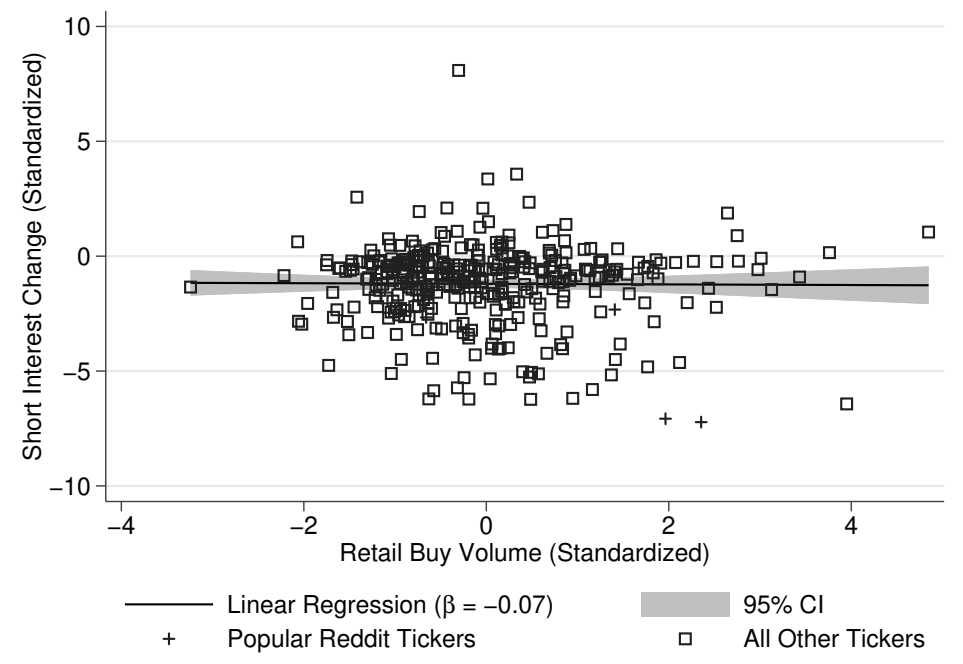

Figure 6: Cross-sectional relationship between retail volume and short interest, January 2021. Changes in both retail purchase volume and change in short interest were calculated as standardized $z$-scores using TAQ and SEC data from January 2015 through January 2021. Each month, we calculate retail purchase volume and short interest; standardized values are demeaned and divided by the sample standard deviation over this period. Tickers which were popular discussion topics on WSB and which are also in the top decile of short interest are indicated with "+", while all other tickers are indicated with " $\square$ ".

to the $y$-axis values, which are overwhelmingly negative, indicating a correlated decline in short interest in that month.

A concluding empirical observation is that the decline in short interest did not show any signs of reverting to its old levels in the six months that followed January 2021 (Figure 18 in Appendix G). This suggests that the short-seller retreat was not just a transient reaction to let the "dust settle." Instead, there appears to have been a shift to a new equilibrium with less shorting.

In summary, the timing of the events suggests that the broad retreat of short sellers could not have been a direct reaction to GameStop losses. In addition, the unremarkable behavior of retail purchases suggests that the numerous stocks that saw a retreat of short interest did not experience outright short squeezes, as GameStop did. Excluding these simple candidate explanations suggests that the retreat of the short sellers was most likely a fearful reaction to some early signs of a shifting market environment. The remainder of the paper proposes a model to explain why short-selling may be susceptible to run-type behavior and retreat 
even as mispricing widens.

\section{Model}

\subsection{Agents: life-cycle and preferences}

Time is continuous and infinite for tractability. To obtain a stationary wealth distribution, we follow Gârleanu and Panageas (2015) and assume that investors continuously arrive ("births") and depart ("deaths") from the economy. Per unit of time a mass $\pi$ of investors arrives, and a mass $\pi$ departs. By the law of large numbers, the population of agents who were born at time $s \leq t$ still remaining at time $t$ is $\pi e^{-\pi(t-s)}$, while the total population is constant and equal to $\int_{-\infty}^{t} \pi e^{-\pi(t-s)} d s=1$. "Births" and "deaths" should be understood as arrivals and departures from the stock market, a point that will become clearer in Section 5. where we introduce multiple stocks.

To introduce trade in equities, we assume that investors have heterogeneous beliefs. For simplicity, a fraction $\nu \in(0,1)$ of investors perceive the correct data-generating process. We refer to them as rational investors (" $R$ " investors). The remaining fraction are overly optimistic (we model this optimism shortly), and we refer to these investors as "I" investors.

For tractability, both investors have logarithmic utilities and their expected discounted utility from consumption is

$$
V_{t}^{i} \equiv E_{t}^{i} \int_{t}^{\infty} e^{-(\rho+\pi)(u-t)} \log \left(c_{u, t}^{i}\right) d u
$$

for $i \in\{I, R\}$, with $\rho$ a discount factor and $c_{u, t}^{i}$ the time- $u$ consumption of an agent of type $i$ born at time $t \leq u$. The notation $E_{t}^{i}$ reflects the different investor beliefs. Because of death, the effective discount rate is $\rho+\pi$.

Before proceeding, we note that while we require heterogeneous beliefs to introduce a motivation for trading, the assumption that one group has correct beliefs helps mostly to save notation and can be easily relaxed. Similarly, the overlapping-generations structure is just a technical device to ensure that no investor type disappears in the long run ${ }^{17}$ Finally,

\footnotetext{
${ }^{17}$ In particular, the lack of inter-generational risk sharing, which is a feature of some of these models, is
} 
in setting up the model we make the (conventional) assumption that agents maximize over both their consumption and portfolio choices, which we introduce shortly. Our model is, however, equivalent to one in which agents delegate their portfolio decisions to professional managers, and managers maximize their clients' expected portfolio (logarithmic) growth according to the managers' beliefs ( $R$ or $I$ ). The investors in our model can therefore be equivalently thought of as institutional investors.

\subsection{Endowments}

In order to support their consumption over their lives, we assume that the arriving investors at time $t$ are equally endowed with shares of new "trees," which arrive at time $t$. Letting $s \leq t$ denote the time of arrival of a tree, we specify its time- $t$ dividends as

$$
D_{t, s}=\delta e^{-\delta(t-s)} D_{t}
$$

where $\delta>0$ captures depreciation and $D_{t}$ follows a geometric Brownian motion with mean $\mu_{D}$ and volatility $\sigma_{D}>0$

$$
\frac{d D_{t}}{D_{t}}=\mu_{D} d t+\sigma_{D} d B_{t}
$$

with $B_{t}$ a standard Brownian motion. Accordingly, the time- $t$ total endowment of this economy is the sum of the endowment produced by all trees born up to to time $t$,

$$
\int_{-\infty}^{t} D_{t, s} d s=\left(\int_{-\infty}^{t} \delta e^{-\delta(t-s)} d s\right) \times D_{t}=D_{t}
$$

The assumption that investors are endowed with shares of newly arriving trees follows Gârleanu et al. (2012) and Panageas (2020). We adopt this assumption rather than introducing labor income (as in Gârleanu and Panageas, 2015 or Gârleanu and Panageas, 2020), because - for the purposes of this paper - labor income would just complicate matters without providing any novel insights.

We note that since the returns of all stocks (across all vintages) are perfectly correlated, not driving any of the results in this paper. 
in effect there is a single, "representative" stock, which is convenient to model. We define the return of this stock as

$$
d R_{t}=\left(\frac{d P_{t, s}+D_{t, s} d t}{P_{t, s}}\right)=\mu_{t} d t+\sigma_{t} d B_{t}
$$

where $\mu_{t}$ and $\sigma_{t}$ are to be determined in equilibrium.

In the real world, shorting frictions are more relevant for a small fraction of stocks rather than the broad stock market. In Section 5 we extend the model to allow for multiple stocks and study the special case in which the shorting frictions are relevant for small stocks only.

\subsection{Beliefs}

The irrational investors are optimistic and believe that the aggregate endowment grows at

the rate $\mu^{I}>\mu_{D}$. Irrational investors hold this optimistic view over their life-time and do not learn ("dogmatic beliefs"). Introducing learning would be a distraction for the purposes of this paper and therefore we omit it.

For future reference, we define

$$
\eta \equiv \frac{\mu^{I}-\mu_{D}}{\sigma_{D}}
$$

\subsection{Dynamic budget constraint and short-selling frictions}

This section embeds stock lending fees (and lending income) into an investor's dynamic budget constraint, which is the novel aspect of our setup. In preparation, we start by defining the return of the market portfolio and introduce a standard assumption on annuitization.

Specifically, as in Gârleanu et al. (2012) and Panageas (2020), the arriving investors support their life-time consumption by selling their firms into the stock market. These firms become part of the market index (the "market portfolio"). Given our assumptions, the market value of arriving companies, $P_{t, t}$, over total market capitalization $P_{t}$ is $\frac{P_{t, t}}{P_{t}}=\delta$.

Letting $P_{t}$ denote aggregate stock market capitalization, the instantaneous return of the 
market portfolio i: 18

$$
\begin{aligned}
d R_{t} & =\underbrace{\frac{d P_{t}}{P_{t}}}_{\begin{array}{c}
\text { Change in } \\
\text { aggregate market cap. }
\end{array}}-\underbrace{\frac{P_{t, t} d t}{P_{t}}}_{\begin{array}{c}
\text { cost to purchase } \\
\text { the new firms }
\end{array}}+\underbrace{\frac{D_{t}}{P_{t}} d t}_{\text {dividend yield }} \\
& =\frac{d P_{t}+D_{t} d t}{P_{t}}-\delta d t .
\end{aligned}
$$

Aside from investing in shares of the market portfolio and (zero net supply) risk-free assets, we follow Blanchard (1985) in assuming that each investor annuitizes her entire wealth (since there are no bequest motives) by pledging it to a competitive insurance company upon death in exchange for receiving an income stream while alive. This income stream is equal to the hazard rate of death, $\pi$, times the wealth of the investor, so that the insurance company breaks even 19

The main departure from a frictionless market is that if investors want to short stocks, they have to pay a lending fee, $f_{t}$. Specifically, letting $W_{t, s}^{i}$ denote the time- $t$ wealth of an investor of type $i$ who was born at time $s \leq t$ and $w_{t, s}^{i}$ the fraction of wealth invested in stocks, the dynamic budget constraint is

$$
d W_{t, s}^{i}=W_{t, s}^{i}\left(r_{t}+\pi+n_{t}+w_{t, s}^{i}\left(\mu_{t}-r_{t}+\lambda_{t, s}^{i}\right)-\frac{c_{t, s}^{i}}{W_{t, s}^{i}}\right) d t+w_{t, s}^{i} W_{t, s}^{i} \sigma_{t} d B_{t}
$$

where $\mu_{t}$ and $\sigma_{t}$ are the equilibrium expected return and volatility (respectively) of a stock investment and $r_{t}$ is the equilibrium interest rate. The non-standard terms in equation (5) are the terms $\lambda_{t, s}^{i}$ and $n_{t}$, which we describe next.

\footnotetext{
${ }^{18}$ For a more detailed derivation, start from $P_{t}=\int_{-\infty}^{t} P_{t, s} d s$. Time-differentiating $\frac{d P_{t}}{P_{t}}$, using Leibniz's rule, and adding $\frac{D_{t}}{P_{t}}=\frac{\int D_{t, s} d s}{P_{t}}$ we obtain

$$
\frac{d P_{t}}{P_{t}}+\frac{D_{t}}{P_{t}} d t=\frac{\int_{-\infty}^{t}\left(d P_{t, s}+D_{t, s} d t\right) d s}{P_{t}}+\frac{P_{t, t}}{P_{t}} d t=\int_{-\infty}^{t}\left(\frac{P_{t, s}}{P_{t}}\right)\left(\frac{d P_{t, s}+D_{t, s} d t}{P_{t, s}}\right) d s+\frac{P_{t, t}}{P_{t}} d t
$$$$
=\int_{-\infty}^{t} w_{t, s}\left(\frac{d P_{t, s}+D_{t, s} d t}{P_{t, s}}\right) d s+\frac{P_{t, t}}{P_{t}} d t=d R_{t}+\frac{P_{t, t}}{P_{t}} d t=d R_{t}+\delta d t,
$$

where $w_{t, s}$ are market-capitalization weights and the equality $d R_{t}=\int_{-\infty}^{t} w_{t, s}\left(\frac{d P_{t, s}+D_{t, s} d t}{P_{t, s}}\right) d s$ constitutes the definition of a portfolio's return.

${ }^{19}$ This conclusion is an implication of the Law of Large Numbers.
} 
The term $\lambda_{t, s}^{i}$ captures the presence of lending fees. The term $\lambda_{t, s}^{i}$ is defined as

$$
\lambda_{t, s}^{i} \equiv \lambda_{t}\left(w_{t, s}^{i}\right) \equiv f_{t} \times\left(1_{\left\{w_{t, s}^{i}<0\right\}}+\tau y_{t} 1_{\left\{w_{t, s}^{i} \geq 0\right\}}\right)
$$

where $y_{t}$ is the fraction of a long portfolio that is lent out by the representative "brokerage house" and $\tau$ is the fraction of the lending fees that accrues to the investor. (We discuss the determination of $y_{t}$ and $\tau$ shortly.) Equation (6) reflects that an investor with a short position $w_{t, s}^{i}<0$ has to pay a proportion $f_{t}$ of the value of her entire short position, $\left|w_{t, s}^{i}\right| W_{t, s}^{i}$, so that the net-of-fee rate of return per dollar shorted is $-\left(\mu_{t}-r_{t}+f_{t}\right) d t-\sigma_{t} d B_{t}$. Similarly, an investor holding a positive position, $w_{t, s}^{i}>0$, obtains a rate of return equal to $\left(\mu_{t}-r_{t}+\tau y_{t} f_{t}\right) d t+\sigma_{t} d B_{t}$ on her stock investments.

Market clearing for share lending requires

$$
y_{t} W_{t}^{+}=W_{t}^{-}
$$

where $W_{t}^{-}$is the value of the aggregate short interest and $W_{t}^{+}$that of the aggregate long position,

$$
\begin{aligned}
& W_{t}^{-} \equiv \sum_{i \in\{I, R\}} \int_{-\infty}^{t}\left|w_{t, s}^{i}\right| W_{t, s}^{i} 1_{\left\{w_{t, s}^{i}<0\right\}} d s \\
& W_{t}^{+} \equiv \sum_{i \in\{I, R\}} \int_{-\infty}^{t} w_{t, s}^{i} W_{t, s}^{i} 1_{\left\{w_{t, s}^{i}>0\right\}} d s .
\end{aligned}
$$

To close the model, we need to specify $n_{t}$ and also a "supply curve" for lending shares, that is, we need to provide a relation between $y_{t}$ and the fee $f_{t}$. To that end, in Appendix A we model the supply curve for lendable shares by introducing competitive firms specializing in servicing either borrowers ("brokers") or lenders ("dealers"). Brokers are faced with a demand from would-be short sellers, while dealers obtain investors' long portfolios. Brokers and dealers are matched pairwise subject to a "labor cost" and engage in bilateral negotiations that result in a lending fee $f_{t}$. In equilibrium, the fee is the same for all shares that are lent, and therefore the total revenue from lending shares equals the fee multiplied by the value of all shares lent. This revenue is shared between the stock lenders (a fraction $\tau$ of 
the lending revenue) and the households as compensation for their labor cost (the remaining $1-\tau$ fraction of lending revenue).

Appendix A derives $\tau$ as a function of parameters governing the search-and-bargaining protocol. In addition, we establish that the lending fee, $f_{t}$, is a non-decreasing function $f_{t}=l\left(y_{t}\right)$ with $l^{\prime}(\cdot) \geq 0$, where $l(\cdot)$ depends on the (exogenous) "technology" of finding a match. From now on, we refer to $l\left(y_{t}\right)$ as the supply curve for lendable shares.

The term $n_{t}$ in equation (5) is the fraction $(1-\tau)$ of the lending revenue that is paid to the households as compensation for their labor cost in operating the matching technology. Denoting aggregate wealth at time $t$ by $W_{t}$, we define

$$
n_{t} \equiv \frac{(1-\tau) f_{t} W_{t}^{-}}{W_{t}}
$$

To better understand (10), use (7) and (10) and aggregate across all households to obtain

$$
\begin{aligned}
f_{t} W_{t}^{-} & =(1-\tau) f_{t} W_{t}^{-}+\tau f_{t} W_{t}^{-} \\
& =n_{t} W_{t}+\tau f_{t} y_{t} W_{t}^{+} .
\end{aligned}
$$

The left hand side of (11) reflects the aggregate lending fees $f_{t} W_{t}^{-}$. The right-hand side reflects the ultimate division of lending income between the households (who obtain a fraction $1-\tau$ of lending, irrespective of their portfolio) and long investors, who obtain a fraction $\tau$ of the lending income.

Equation (11) shows that share lending does not result in any loss of aggregate resources: All payments made by investors with short positions are received either by investors with long positions or by brokerage firms, who rebate them to the household sector.

\subsection{Equilibrium definition}

Equilibrium in the lending market requires that the lending fee be such that the supply of lendable shares $y_{t} W_{t}^{+}=l^{-1}\left(f_{t}\right) W_{t}^{+}$is equal to the demanded short interest, $W_{t}^{-}$(equation (7))

The rest of the equilibrium definition is standard. We require that investors $I$ and $R$ 
maximize (1) over $c_{t, s}^{i}$ and $w_{t, s}^{i}$ subject to the budget constraint (5), and $\mu_{t}, r_{t}$, and $\sigma_{t}$ are such that the bond market clears, $\sum_{i \in\{I, R\}} \int_{-\infty}^{t} \nu^{i}\left(1-w_{t, s}^{i}\right) W_{t, s}^{i} d s=0$, the stock market clears, $\sum_{i \in\{I, R\}} \int_{-\infty}^{t} \nu^{i} w_{t, s}^{i} W_{t, s}^{i} d s=P_{t}$, and the goods market clears, $\sum_{i \in\{I, R\}} \int_{-\infty}^{t} \nu^{i} c_{t, s}^{i} d s=D_{t}$. By Walras' Law, market clearing of the bond market implies stock market clearing and vice versa, and accordingly the asset-market clearing requirements can be written equivalently as $W_{t}=\sum_{i \in\{I, R\}} \int_{-\infty}^{t} \nu^{i} W_{t, s}^{i} d s=P_{t}$.

For future reference, we note that stock market clearing requires that $y_{t}<1$ :

$$
y_{t}=\frac{W_{t}^{-}}{W_{t}^{+}}=\frac{W_{t}^{-}}{P_{t}+W_{t}^{-}}<1
$$

\section{Analysis}

We analyze the model in two steps. First, we consider a special parametric case that allows us to characterize all equilibrium quantities in closed form. The special case we analyze is the "elastic supply" case, that is, the limiting case where the supply of lendable shares is horizontal at some level $l\left(y_{t}\right)=\varphi$. (As we explain in Appendix A, this special case corresponds to a linear specification for the cost of lending out shares.) In Appendix $\mathrm{D}$ we repeat the analysis for increasing functions $l\left(y_{t}\right)$ and show how the key results extend to this more general case.

\subsection{Optimal portfolio and consumption}

For a log investor the wealth-to-consumption ratio is constant and equal to

$$
\frac{c_{t, s}^{i}}{W_{t, s}^{i}}=\rho+\pi
$$

Another convenient property of logarithmic utility is that the portfolio is myopic and maximizes the logarithmic growth rate of an investor's wealth, under the investor's beliefs,

$$
w_{t, s}^{i}=\arg \max _{w}\left\{w\left(\mu_{t}+\eta \sigma_{t} 1_{\{i=I\}}-r_{t}+\lambda_{t}(w)\right)-\frac{1}{2}\left(w \sigma_{t}\right)^{2}\right\}
$$

where $1_{\{i=I\}}$ is an indicator function taking the value one when $i=I$ and zero otherwise. 


\section{Letting}

$$
\widehat{\mu}_{t}^{i} \equiv \mu_{t}+\eta \sigma_{t} 1_{\{i=I\}}
$$

denote the expected return on the stock as perceived by investor $i \in\{I, R\}$, the optimal portfolio is

$$
w_{t, s}^{i}= \begin{cases}\frac{\widehat{\mu}_{t}^{i}-r_{t}+f_{t}}{\sigma_{t}^{2}} & \text { if } \widehat{\mu}_{t}^{i}-r_{t}+f_{t}<0 \\ \frac{\widehat{\mu}_{t}^{i}-r_{t}+\tau f_{t} y_{t}}{\sigma_{t}^{2}} & \text { if } \widehat{\mu}_{t}^{i}-r_{t}+\tau f_{t} y_{t}>0 . \\ 0 & \text { otherwise }\end{cases}
$$

By inspection, the optimal portfolios do not depend on the cohort $s$, only on the type of investor $i \in\{I, R\}$. Therefore, from now on we drop the subscript $s$ and write $w_{t}^{R}$ and $w_{t}^{I}$.

One straightforward implication of equation (14) is that if investor $R$ is actively shorting $\left(w_{t}^{R}<0\right)$ then it must be the case that the excess rate of return per dollar shorted is positive, even after netting out the fee $f_{t} 20$

\subsection{Equilibrium}

It is useful to start by defining the the wealth-weight $\omega_{t}^{i}$

$$
\omega_{t}^{i} \equiv \frac{\nu^{i} \int_{-\infty}^{t} \pi e^{-\pi(t-s)} W_{t, s}^{i} d s}{W_{t}}
$$

The goods market and stock market clearing requirements imply

$$
\begin{aligned}
D_{t} & =\sum_{i \in\{I, R\}} \int_{-\infty}^{t} \nu^{i} \pi e^{-\pi(t-s)} c_{t, s}^{i} d s=(\rho+\pi) \sum_{i \in\{I, R\}} \int_{-\infty}^{t} \nu^{i} \pi e^{-\pi(t-s)} W_{t, s}^{i} d s \\
& =(\rho+\pi) W_{t}=(\rho+\pi) P_{t} .
\end{aligned}
$$

\footnotetext{
${ }^{20}$ Evaluating (14) with $i=R$, assuming that $w_{t}^{R}<0$, and re-arranging leads to $-\left(\mu_{t}-r-f_{t}\right)=$ $-\left(\widehat{\mu}_{t}^{R}-r-f_{t}\right)=-w_{t}^{R} \sigma_{t}^{2}>0$. The term $-w_{t}^{R} \sigma_{t}^{2}$, which corresponds to the absolute value of the covariance of the stock's return with the short seller's portfolio, is the risk compensation to the short seller for taking a short position.
} 
Taking $\operatorname{logarithms}$ gives $d \log D_{t}=d \log P_{t}$ and therefore the stock market volatility equals $\sigma=\sigma_{D}$. The implication of a constant stock volatility is convenient for obtaining closedform solutions. In Section 5 we discuss extensions of the model that allow for a time-varying price-dividend ratio and volatility by introducing multiple stocks.

Applying Itô's Lemma to (15) and using (5) and (16) yields

$$
d \omega_{t}^{i}=\mu_{t}^{i} d t+\sigma_{t}^{i} d B_{t}
$$

where

$$
\begin{aligned}
\sigma_{t}^{i} & =\omega_{t}^{i}\left(w_{t}^{i}-1\right) \sigma_{D} \\
\mu_{t}^{i} & =\omega_{t}^{i}\left(-\mu_{D}+\sigma_{D}^{2}-\pi+r_{t}-\rho+w_{t}^{i}\left(\mu_{t}-r_{t}+s_{t}^{i}\right)-w_{t}^{i} \sigma_{D}^{2}\right)+\nu^{i} \delta
\end{aligned}
$$

The market clearing requirement $\sum_{i \in\{I, R\}} \omega_{t}^{i}=1$ implies $\sum_{i \in\{I, R\}} d \omega_{t}^{i}=0$ and therefore $\sum_{i \in\{I, R\}} \sigma_{t}^{i}=0$ and $\sum_{i \in\{I, R\}} \mu_{t}^{i}=0$. To simplify notation, we let $\omega_{t} \equiv \omega_{t}^{R}$. As mentioned earlier, in an effort to obtain a tractable solution, we assume that the supply of lendable shares is perfectly elastic at the rate $\varphi$ :

Assumption $1 l(y)=\varphi>0$.

We maintain this assumption to develop intuition. In Appendix D we generalize the results to an upward-sloping supply function $l(\cdot)$, so that the lending fee is increasing with short interest.

In preparation for the description of the equilibrium, we start with the following definition and assumptions on the parameters.

\section{Definition 1 Let}

$$
\omega_{1}^{*} \equiv 1-\frac{\sigma_{D}}{\eta-\frac{\varphi}{\sigma_{D}}} .
$$

and

$$
F(\omega) \equiv\left(\sigma_{D}-\omega\left((1+\tau) \frac{\varphi}{\sigma_{D}}-\eta\right)\right)^{2}-4 \tau \frac{\omega^{2}}{1-\omega} \frac{\varphi}{\sigma_{D}}\left(\sigma_{D}+(1-\omega)\left(\frac{\varphi}{\sigma_{D}}-\eta\right)\right)
$$


Assumption 2 Assume that $\eta, \varphi, \sigma_{D}$, and $\tau$ are such that

$$
\begin{gathered}
(1+\tau) \frac{\varphi}{\sigma_{D}}>\eta>\frac{\varphi}{\sigma_{D}}, \\
\omega_{1}^{*}>\frac{\sigma_{D}}{(1+\tau) \frac{\varphi}{\sigma_{D}}-\eta}>0,
\end{gathered}
$$

and $F(\omega)$ has a unique root in the interval $(0,1)$, denoted by $\omega_{2}^{*}$.

The following proposition asserts that the set of parameters $\eta, \varphi, \sigma_{D}$, and $\tau$ that satisfy Assumption 2 is non-empty.

Proposition 1 There exists an open set of positive values $\eta, \varphi, \sigma_{D}$, and $\tau$ that jointly satisfy Assumption 2.

The next proposition describes the equilibria in our economy.

Proposition 2 Suppose that Assumption 2 holds. Then $\omega_{2}^{*}>\omega_{1}^{*}$ and the equilibria in this economy can be described as follows.

i) If $\omega_{t} \in\left(\omega_{2}^{*}, 1\right]$ there is no short-selling in equilibrium. The equilibrium is unique and the Sharpe ratio $\kappa_{t} \equiv \frac{\mu_{t}-r_{t}}{\sigma_{D}}$ is given by

$$
\kappa_{t}=\left\{\begin{array}{ll}
\sigma_{D}-\left(1-\omega_{t}\right) \eta & \text { if } \omega_{t}>1-\frac{\sigma_{D}}{\eta} \\
\frac{\sigma_{D}}{1-\omega_{t}}-\eta & \text { if } \omega_{t} \in\left(\omega_{2}^{*}, 1-\frac{\sigma_{D}}{\eta}\right]
\end{array} .\right.
$$

ii) If $\omega_{t} \in\left[\omega_{1}^{*}, \omega_{2}^{*}\right]$, then there are three equilibria. The first equilibrium continues to be given by (23) and involves no short-selling. The second and third equilibria involve shorting and the ratio of shorted-to-lendable shares $y_{t}$ corresponds to the two roots $y^{+}$and $y^{-}$of the quadratic equation

$$
y\left(\eta+\frac{\sigma_{D}}{\omega_{t}}-\frac{\varphi}{\sigma_{D}}(1-\tau y)\right)-\left(\eta-\frac{\sigma_{D}}{1-\omega_{t}}-\frac{\varphi}{\sigma_{D}}(1-\tau y)\right)=0
$$

which has two real roots $y^{+}$and $y^{-}$in the interval $(0,1)$. The Sharpe ratio in the equilibria associated with $y^{+}$and $\left.y^{-}\right)$are

$$
\kappa_{t}^{ \pm}=\sigma_{D}-\left(1-\omega_{t}\right) \eta-\frac{\varphi}{\sigma_{D}}\left(\omega_{t}+\tau y^{ \pm}\left(1-\omega_{t}\right)\right)
$$




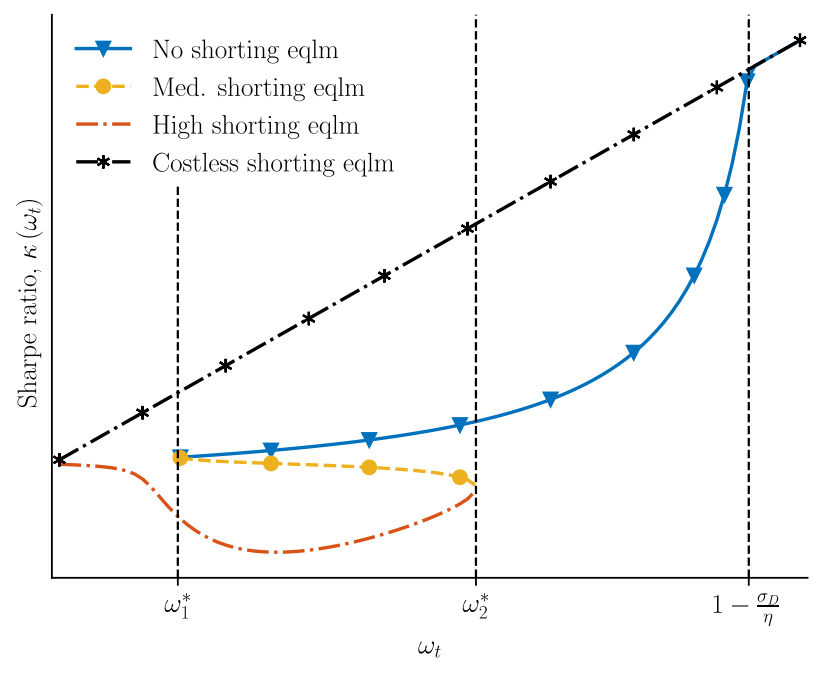

Figure 7: An illustration of all possible equilibrium values of the Sharpe ratio, depending on the value of the wealth ratio $\omega_{t}$, as stated in Proposition 2.

iii) If $\omega_{t} \in\left[0, \omega_{1}^{*}\right)$, then the equilibrium is unique and involves shorting. In this case only the larger of the two roots $\left(y^{+}\right)$of equation (24) lies in the interval $(0,1)$, and the unique equilibrium Sharpe ratio is given by $\kappa^{+}$.

In all three cases the interest rate is given by

$$
r_{t}=\rho+\pi+\mu_{D}-\delta-\kappa_{t} \sigma_{D}
$$

Additionally, because $\kappa_{t}, r_{t}$, and $y_{t}$ are functions of $\omega_{t}$, equations (14), (17), and (18) imply that $\mu_{t}^{R}$ and $\sigma_{t}^{R}$ are functions of $\omega_{t}$ and hence the equilibrium is Markov in $\omega_{t}$.

Figure 7 illustrates Proposition 2. The figure plots $\kappa\left(\omega_{t}\right)$, the Sharpe ratio, as a function of the wealth share of rational agents. As a benchmark, the line labeled "Costless shorting eqlm" depicts $\sigma_{D}-\left(1-\omega_{t}\right) \eta$, i.e., the Sharpe ratio that would obtain in this economy in the absence of any shorting frictions $(\varphi=0)$. The curve "No shorting eqlm" depicts the Sharpe ratio in the equilibrium that involves no shorting for the values of $\omega_{t}$ that such an equilibrium exists. Similarly for the curves "Med. shorting eqlm" and "High shorting eqlm," which depict equilibria with shorting for the values of $\omega_{t}$ that permit such equilibria. To expedite the exposition of the results, we postpone a discussion of the quantitative implications of the 


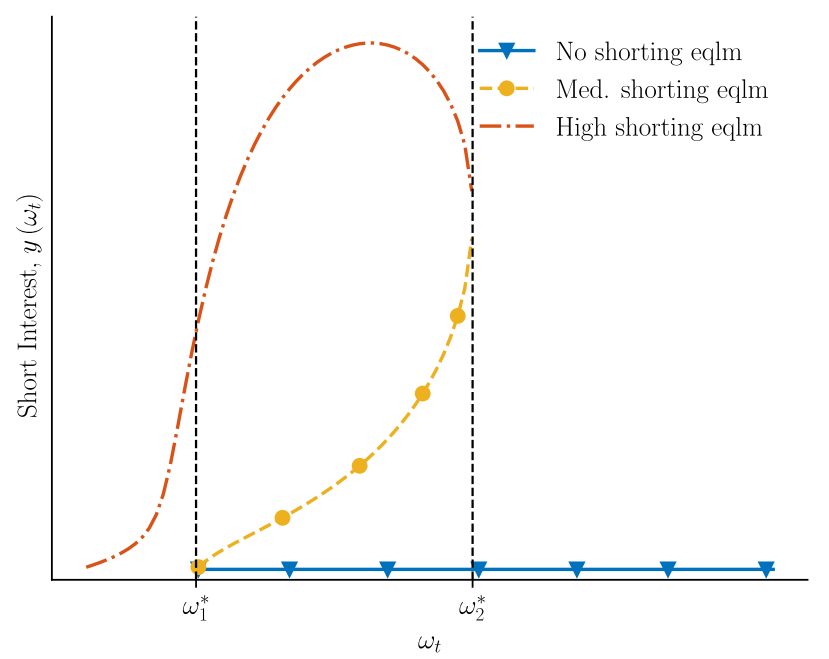

Figure 8: The ratio of shorted-to-lendable shares, $y_{t}$, in all of the equilibria as a function of $\omega_{t}$.

model until Section 5.2. The figures in the current section are meant to illustrate qualitative properties of the model.

The figure shows that when $\omega_{t}$ is larger than $1-\frac{\sigma_{D}}{\eta}$ the lines "Zero shorting cost" and "No shorting eqlm" coincide, reflecting that all investors invest strictly positive amounts in the stock market in this region.

When $\omega_{t}$ becomes smaller than $1-\frac{\sigma_{D}}{\eta}$ (but larger than $\omega_{2}^{*}$ ), the rational investor puts zero weight on stocks, but the shorting fee $\varphi$ deters her from actively short-selling. Since only the irrational investor is marginal in financial markets, the lines "Zero shorting cost" and "No shorting eqlm." deviate from each other when $\omega_{t}<1-\frac{\sigma_{D}}{\eta}$. In this region the magnitude of the lending fee, $\varphi$, does not impact the Sharpe ratio directly (only by deterring the $R$ investors from shorting).

If $\omega_{t}$ becomes smaller than $\omega_{2}^{*}$ (but larger than $\omega_{1}^{*}$ ) the economy exhibits three equilibria. In the first equilibrium, there is still no shorting. In the second and third, there is active shorting by the rational investor. Across these three equilibria, the higher the extent of shorting, the lower the Sharpe ratio. This is illustrated in Figure 8. If $\omega_{t}$ becomes smaller than $\omega_{1}^{*}$, then the equilibrium becomes unique and involves shorting ${ }^{21}$

\footnotetext{
${ }^{21}$ To see why there can be no equilibrium without shorting when $\omega_{t}<\omega_{1}^{*}$, assume otherwise. Indeed
} 
Several features of Figure 7 are noteworthy. First, the Sharpe ratio is always (weakly) lower than it would be in the absence of lending fees, even if investor $R$ is not actively shorting shares, but is only investing in bonds.

Second, the presence of a region where multiple equilibria co-exist is not a very common feature of asset pricing models, especially when there is only one good and one positivesupply asset. To better understand the source of this multiplicity, it is useful to provide a concise derivation of the key statements in Proposition 1.

Specifically, suppose that we consider equilibria that involve active shorting $\left(w_{t}^{R}<0\right)$. In such equilibria, the optimal portfolio holdings can be expressed as

$$
\begin{aligned}
w_{t}^{R} & =\frac{\kappa_{t}+\frac{\varphi}{\sigma_{D}}}{\sigma_{D}} \\
w_{t}^{I} & =\frac{\kappa_{t}+\eta+\frac{\varphi}{\sigma_{D}} \tau y_{t}}{\sigma_{D}}
\end{aligned}
$$

while asset-market clearing requires

$$
\omega_{t} w_{t}^{R}+\left(1-\omega_{t}\right) w_{t}^{I}=1
$$

Combining equations (27)-29) leads to

$$
\kappa_{t}=\sigma_{D}-\left(1-\omega_{t}\right) \eta-\frac{\varphi}{\sigma_{D}}\left(\omega_{t}+\tau y_{t}\left(1-\omega_{t}\right)\right)
$$

which is equation (25) of Proposition 1. Note that the partial derivative of $\kappa_{t}$ with respect to $y_{t}$ is negative. This is intuitive: A higher value of $y_{t}$ increases the effective rate of return to (long-portfolio) stock holders ( $I$ investors). The increased appetite by $I$ investors to hold long positions lowers the Sharpe ratio. (Phrased differently, the absolute value of the Sharpe ratio increases, since the Sharpe ratio is negative when $w_{t}^{R}<0$.)

assume that the $R$ investor holds zero stocks and is not marginal in the stock market $\left(w_{t}^{R}=0\right)$. The market clearing requirement, $\omega_{t} w_{t}^{R}+\left(1-\omega_{t}\right) w_{t}^{I}=1$, along with $w_{t}^{I}=\frac{\kappa_{t}+\eta}{\sigma_{D}}$ implies that the Sharpe ratio would be $\kappa_{t}=\frac{\sigma_{D}}{1-\omega_{t}}-\eta$. Under this supposition, it would therefore be the case that $\mu_{t}-r+\varphi=$ $\sigma_{D}\left(\kappa_{t}+\frac{\varphi}{\sigma^{D}}\right)=\sigma_{D}\left(\frac{\sigma_{D}}{1-\omega_{t}}-\eta+\frac{\varphi}{\sigma^{D}}\right)<0$, where the inequality follows from $\omega_{t}<\omega_{1}^{*}$. Because $\mu_{t}-r+\varphi<0$, equation (14) implies that the $R$ investor would want to short the market, contradicting the assumption that she is optimally holding zero stocks. 
This lowering of the Sharpe ratio strengthens the short-sellers' appetite to borrow the stock and short it. In equilibrium, the increased shorting demand raises the ratio of shortedto-lendable shares, $y_{t}$, increasing the effective return to $I$ investors, which further reduces the Sharpe ratio, etc.

These self-reinforcing effects are the root cause of the multiple equilibria. The easiest way to see this is by completing the computation of the Sharpe ratio, which requires us to determine the value of $y_{t}$ that clears the lending market. Indeed, in any equilibrium involving $w_{t}^{R}<0$ and $w_{t}^{I}>0$ we must have

$$
y_{t}=\frac{W_{t}^{-}}{W_{t}^{+}}=\frac{-w_{t}^{R} W_{t}^{R}}{w_{t}^{I} W_{t}^{I}}=-\frac{w_{t}^{R}}{w_{t}^{I}} \times \frac{\omega_{t}}{1-\omega_{t}} .
$$

Using 27 to compute the ratio $\frac{w_{t}^{R}}{w_{t}^{I}}$ gives

$$
\begin{aligned}
y_{t} & =-\frac{\kappa_{t}+\frac{\varphi}{\sigma_{D}}}{\kappa_{t}+\eta^{I}+\frac{\varphi}{\sigma_{D}} \tau y_{t}} \times \frac{\omega_{t}}{1-\omega_{t}} \\
& =\frac{\eta-\frac{\sigma_{D}}{1-\omega_{t}}-\frac{\varphi}{\sigma_{D}} \tau\left(1-y_{t}\right)}{\eta+\frac{\sigma_{D}}{\omega_{t}}-\frac{\varphi}{\sigma_{D}} \tau\left(1-y_{t}\right)},
\end{aligned}
$$

where the last line follows from (30) after collecting terms and simplifying. Rearranging (32) gives the quadratic equation (24), which is the key equation of Proposition 1 . The rest of the proposition is devoted to studying this quadratic equation and confirming that its roots correspond to valid equilibria.

While equation (32) is particularly simple to analyze, the multiplicity of equilibria does not hinge on assuming that the supply curve $l\left(y_{t}\right)$ is constant at the level $\varphi$, as we show in Appendix D.

The intuition behind the multiplicity of equilibria is contained in equation (32). For a given wealth distribution and belief discrepancy, a higher $y_{t}$ makes long investors content with holding the same positive position at a lower equilibrium Sharpe ratio. This negative relation between the Sharpe ratio and $y_{t}$ is responsible for equilibrium multiplicity: For instance, if something prompts rational investors to abandon their short positions, the resulting reduction in lending income requires a higher Sharpe ratio to compensate the long 
investors and clear the market. But this rise in the Sharpe ratio reinforces the incentive of short sellers to abandon their positions, which further lowers lending income, and further raises the Sharpe ratio, etc., until the market settles on a new equilibrium with (possibly zero) short interest.

Remark 1 The fact that there are three equilibria, one of which features no shorting, is an implication of there being only two types of agents in the model. With more than two types of agents, more than three equilibria can obtain. Also, in the case of multiple equilibria, all of the equilibria can involve strictly positive short interest. We elaborate on these points in Appendix B.

\section{Properties of the Equilibria}

In Section 4.1 we show that equilibria with high shorting are beneficial for $R$ investors. This implies that the worst possible outcome for $R$ investors is for markets to coordinate on the equilibrium that deters them from short selling. Sections 4.2 and 4.3 discuss some broader implications of the model that are unrelated to coordination, but help further illustrate the model's key intuitions. Specifically, we perform comparative statics with respect to changes in $\varphi$, which capture shifts in the supply of lendable shares. Section 4.2 shows how marginal changes in the supply curve can lead to discontinuous drops in short interest. Section 4.3 shows that exogenous shifts in the supply of lendable shares may impact lending fees and short interest but have a muted impact on equilibrium expected returns.

\subsection{Dynamics of the wealth shares}

The three equilibria we identified above have different implications for the dynamics of the wealth shares of $R$ investors. The next proposition shows that both the drift rate $\mu_{t}^{R}\left(\omega_{t}\right)$ of the wealth share of type $R$ investors and the expected logarithmic growth rate of their wealth are higher in equilibria that feature higher short interest $y_{t}$.

Proposition 3 For a fixed wealth share of the $R$-agents, $\omega_{t}$, consider two equilibria $A$ and $B$ with the following properties: 


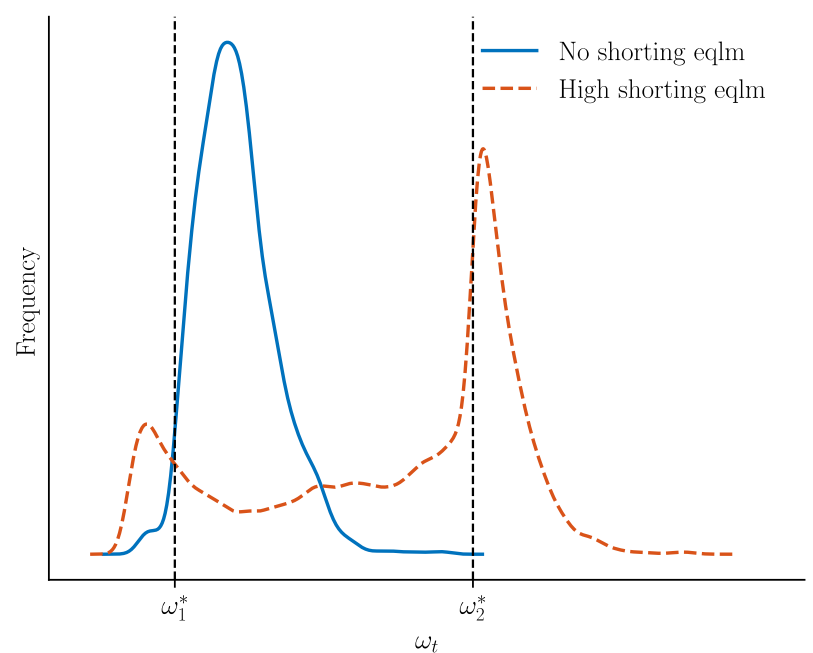

Figure 9: An illustration of Proposition 3 . Simulating the model for the case in which market participants coordinate on the "high shorting" (respectively, "no shorting") equilibrium, the figure depicts the stationary distribution of the wealth share of the rational investor, $\omega_{t}$, for the economy of Figure 7 .

1. $w_{t}^{R} \leq 0$ in both equilibria $A$ and $B$.

2. $y_{t}^{B}>y_{t}^{A}$ (and accordingly $\kappa_{t}^{B}<\kappa_{t}^{A}$ ).

Then the drift of investor $R$ 's wealth share in equilibrium $i \in\{A, B\}, \mu_{t}^{R, i}\left(\omega_{t}\right)$, satisfies $\mu_{t}^{R, B}\left(\omega_{t}\right)>\mu_{t}^{R, A}\left(\omega_{t}\right)$

In addition, the drift of the logarithmic growth rate of investor $R$, defined as

$$
g_{t}^{R} \equiv r_{t}+\max _{w^{R} \leq 0}\left\{w_{t}^{R}\left(\kappa_{t} \sigma_{D}+\varphi\right)-\frac{1}{2}\left(w_{t}^{R} \sigma_{D}\right)^{2}\right\}-(\rho+\pi)
$$

is higher in equilibrium $B$ than in equilibrium $A$, i.e., $g_{t}^{R, B}\left(\omega_{t}\right)>g_{t}^{R, A}\left(\omega_{t}\right)$.

Figure 9 provides an illustration of Proposition 3 . The figure shows the stationary distribution of $\omega_{t}$ in the equilibrium associated with no shorting for values $\omega_{t} \in\left(\omega_{1}^{*}, \omega_{2}^{*}\right)$ and in the equilibrium associated with the highest shorting, $y^{+}\left(\omega_{t}\right)$, for $\omega_{t} \in\left(\omega_{1}^{*}, \omega_{2}^{*}\right)$. The figure shows that the distribution of $\omega_{t}$ has a higher stationary mean in the second equilibrium 
rather than in the first equilibrium. This is consistent with Proposition 3, which asserts a higher (logarithmic) growth rate for the wealth of $R$ investors in the second equilibrium.

The comparatively higher probability mass of larger values of $\omega_{t}$ in the equilibrium that features shorting implies that there are two competing effects on the stationary mean of the Sharpe ratio $\kappa_{t}$. On the one hand - for a fixed $\omega_{t}$ - the Sharpe ratio is lower in equilibria featuring comparatively higher short selling. On the other hand, low values of $\omega_{t}$ become less likely in equilibria with comparatively more shorting activity. The first effect tends to lower the stationary mean of the Sharpe ratio in equilibria with comparatively higher shorting, the second effect tends to raise it. The overall effect on the stationary value of the Sharpe ratio is ambiguous. We revisit this issue in Section 5, when we discuss an extension of the model that allows for multiple stocks, a time-varying price-dividend ratio, and endogenous exit of $R$ investors. Specifically, that section shows that $R$ investors tend to exit the market for a stock when it is not profitable to short, thus resulting in low values of $\omega_{t}$ in the noshorting equilibrium (and therefore low values of the Sharpe ratio and high values of the price-dividend ratio).

\subsection{The instability of short interest}

Besides the sensitivity that emanates from demand-side coordination, our model also implies that small shifts in the supply of lendable shares can lead to discontinuous changes in equilibrium short interest.

Lemma $1 \frac{d \omega_{2}^{*}}{d \varphi}<0$

Lemma 1 states that an increase in $\varphi$ lowers the range of values $\omega_{t}$ that are associated with multiple equilibria. By implication, if, say, a given company can take some action to to reduce the supply of its lendable shares (resulting in an increase in $\varphi$ ), this can lead to a discontinuous change of short interest from a positive value to 0 if $\omega_{t}$ is close to $\omega_{2}^{*}$.

Figure 10 illustrates the effects of an increase in $\varphi$ on the equilibrium Sharpe ratio $\kappa\left(\omega_{t}\right)$. The black curves correspond to the original value of $\varphi$, while the red curves to the higher one. The figure shows that points such as $A$ and $B$, which represent equilibria with positive short interest for the original value of $\varphi$, stop being equilibria if $\varphi$ increases. 


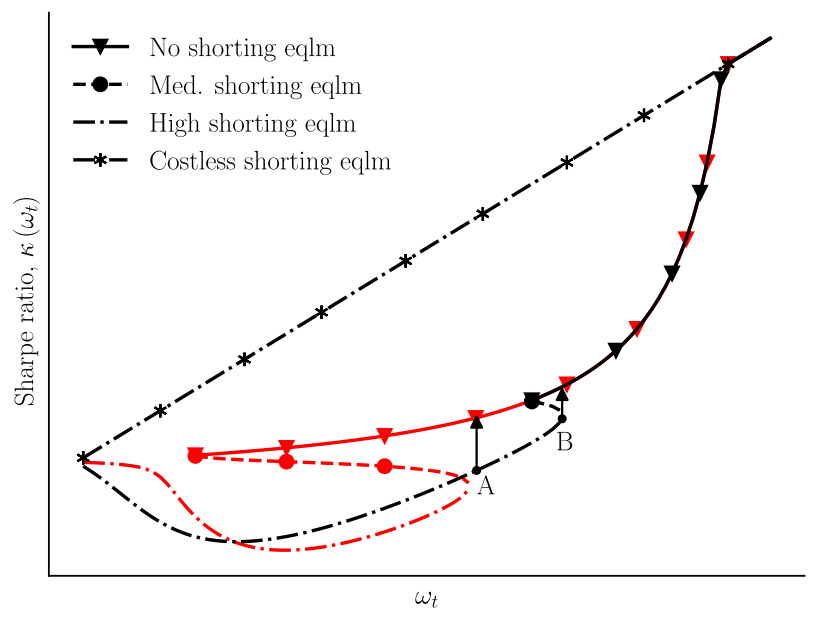

Figure 10: The red curve corresponds to a higher fee $\varphi$. Points $A$ and $B$, which are equilibrium points for the low value of $\varphi$, would entail a discontinuous rise in the Sharpe ratio when $\varphi$ increases.

\subsection{The ambiguous relation between Sharpe ratio and short in- terest}

It would seem natural to expect that an increase in the supply of lendable shares (a reduction in $\varphi$ ) raises the Sharpe ratio, as it incentivizes short sellers to short the stock and thus lowers the absolute value of the (negative) Sharpe ratio. In our model, this result need not obtain. Depending on the equilibrium, there is no unambiguous relation between the Sharpe ratio and shorting costs. This may be one of the reasons why the empirical literature finds that randomized increases in lendable shares affect short interest and lending rates but not excess returns.

The following proposition illustrates the novel implications of the model by focusing on the case of small $\omega_{t}$.

Proposition 4 Assume that the equilibrium involves a positive short interest. In the equilibrium associated with $y^{+}$(which is the unique equilibrium if $\omega_{t}<\omega_{1}^{*}$ ), it holds that, for 
sufficiently small $\omega_{t}$,

$$
\frac{d \kappa}{d \varphi}>0
$$

In the equilibrium associated with $y^{-}$, for any value of $\omega_{t}$,

$$
\frac{d \kappa}{d \varphi}<0
$$

Equation (34) in Proposition 4 appears counterintuitive. The explanation is that decreasing $\varphi$ has two opposing effects. Inspection of equation (30) shows that a decline in $\varphi$ has the direct effect of raising $\kappa_{t}$; however, since $y_{t}$ is endogenous, the decline in $\varphi$ also increases $y_{t}$, which - for a given $\varphi$ - has the effect of lowering $\kappa_{t}$. Therefore, it is possible that a decline in $\varphi$ (say, because of an exogenous change in the cost of supplying shares) lowers the fee $f_{t}$ and increases the short interest $y_{t}$, but leaves the expected return on the stock unchanged. This is consistent with the empirical findings of Kaplan et al. (2013).

Figure 10 illustrates that an increase in $\varphi$ could either raise or lower $\kappa_{t}\left(\omega_{t}\right)$, depending on the equilibrium and on whether $\omega_{t}$ is large or small.

\section{Multiple Risky Assets and Time-Varying Price-Dividend Ratio}

In the baseline model, the price-dividend ratio and the volatility of the stock market are both constant. This is an implication of a) logarithmic utility over intermediate consumption (which implies a constant wealth-to-consumption ratio) and b) a single asset in positive net supply. As is typical of models with similar setups, fluctuations in the interest rate offset the fluctuations of the risk premium, thus rendering the overall discount rate - and by implication the price-dividend ratio 22 - constant.

We next present a version of the model that features multiple risky assets and, by implication, a time-varying price-dividend ratio. After extending Proposition 2 to allow for

\footnotetext{
${ }^{22}$ Note also that the expected dividend growth is constant.
} 
multiple risky assets — a result of independent interest — we consider a limiting case of the multi-asset model that permits simple computations. Specifically, we study the limit in which there is a "small" stock subject to shorting costs and a "large" stock that can be shorted costlessly. In that limit, only the endowment of the large stock matters for the interest rate and thus the price-dividend ratio of the small stock is time-varying and reflects variations in its risk premium. As a byproduct, we can study how the price of the asset changes when the nature of the equilibrium shifts, a phenomenon that lies at the core of the paper. In particular, we show that moving to an equilibrium with low shorting can be accompanied by a price increase, driven by the shift in the composition of the investors for the asset: would-be shorters are driven away, and the price is set predominantly by optimists.

\subsection{Multiple risky assets}

In this section we introduce an additional Lucas tree (stock 2) to our baseline model, which is not subject to any trading frictions, and comprises a potentially large part of the total market capitalization. We continue to assume that borrowing stock 1, which now comprises only (a possibly small) part of the market capitalization, requires lending fees, as in the baseline model.

We make one more convenient and realistic assumption. Specifically, while all investors participate in the markets for stock 2 and the risk-free asset, only a fraction of investors pays any "attention" to stock 1 . The remaining fraction of investors simply optimize their portfolio over the risk-free asset and stock 2 and assign zero weight to stock 1 . This assumption is in the spirit of Robert Merton's "limited recognition hypothesis," the idea that only a fraction of investors actively trade in some smaller stocks. .

Because stock 1 is no longer the only positive-supply asset, consumption-market clearing no longer implies a constant price-to-dividend ratio for stock 1, and a full analytical solution of the model is no longer available. However, we can still provide an analytic "CAPM-style" formula. ${ }^{23}$ which constitutes a natural extension of the results of Proposition 2.

To start, we assume that in equilibrium the returns on stocks 1 and 2 follow a (possibly

\footnotetext{
${ }^{23}$ By CAPM-style formula we mean that the formula provides a connection between expected excess returns and the covariance matrix of returns.
} 
correlated) vector diffusion process of the form

$$
\begin{aligned}
& d R_{1, t}=\mu_{1, t} d t+\sigma_{1, t} d B_{1, t}+b_{t} \sigma_{2, t} d B_{2, t} \\
& d R_{2, t}=\mu_{2, t} d t+\sigma_{2, t} d B_{2, t}
\end{aligned}
$$

where $B_{1, t}$ and $B_{2, t}$ are independent Brownian motions, $\mu_{1, t}$ and $\mu_{2, t}$ are the expected excess returns of the two stocks, and

$$
\sigma_{t} \equiv\left[\begin{array}{cc}
\sigma_{1, t} & b_{t} \sigma_{2, t} \\
0 & \sigma_{2, t}
\end{array}\right]
$$

is a matrix capturing the loadings of the two stocks on the two Brownian motions. We assume that investors $I$ believe that Brownian motion 1 follows the dynamics ${ }^{24} d B_{1, t}+\eta d t$, while no investor has any belief distortions pertaining to Brownian motion 2. We let $\vec{m}_{t}$ denote the vector of market-capitalization weights of the two stocks, and $m_{j, t}, j \in\{1,2\}$, its entries.

From now on we use $W_{t}^{i}$ to denote the wealth of all agents of type $i$ that participate in market 1 and define $\omega_{t}^{i} \equiv W_{t}^{i} /\left(W_{t}^{R}+W_{t}^{I}\right)$. Letting $\widehat{\omega}_{t}$ denote the wealth share of the investors who actively participate in the market for stock 1, the market clearing condition is

$$
\widehat{\omega}_{t} \sum_{i \in\{I, R\}} \omega_{t} \vec{w}_{t}^{i}+\left(1-\widehat{\omega}_{t}\right)\left[\begin{array}{c}
0 \\
\widehat{w}_{2, t}
\end{array}\right]=\vec{m}_{t},
$$

where $\widehat{w}_{2, t}=\frac{\mu_{2, t}-r_{t}}{\sigma_{2, t}^{2}}$ is the optimal portfolio holding of stock 2 by investors who don't participate in stock 1 , and $\vec{w}_{t}^{i}$ is the vector of portfolio holdings of an investor $i \in\{I, R\}$ that is active in the market for stock 1. The market clearning condition (38) leads to the following result.

\footnotetext{
${ }^{24}$ More formally, the Radon-Nikodym derivative of the true probability measure with respect to the subjective one is given by

$$
Z_{t}^{I} \equiv e^{-\frac{\eta^{2}}{2} t+\eta B_{1, t}}
$$
}


Proposition 5 Define $\kappa_{1, t}=\frac{\left(\mu_{1, t}-r\right)-b_{t}\left(\mu_{2, t}-r\right)}{\sigma_{1, t}}$ as the Sharpe ratio of a portfolio that invests 1 unit in asset 1 and shorts $b_{t}$ units of asset 2. Let $\widetilde{m}_{1, t} \equiv \frac{m_{1, t}}{\widehat{\omega}_{t}}$.

In an equilibrium with shorting in asset $1\left(y_{t}>0\right), y_{t}$ is given by the root(s) of the quadratic equation

$$
0=y\left(\eta+\frac{\tilde{m}_{1, t}}{\omega_{t}} \sigma_{1, t}-\frac{\varphi}{\sigma_{1, t}}(1-\tau y)\right)-\left(\eta-\frac{\tilde{m}_{1, t}}{1-\omega_{t}} \sigma_{1, t}-\frac{\varphi}{\sigma_{1, t}}(1-\tau y)\right)
$$

that lie(s) in the interval $[0,1)$, and the Sharpe ratio is given by

$$
\kappa_{1, t}=\widetilde{m}_{1, t} \sigma_{1, t}-\left(1-\omega_{t}\right) \eta-\frac{\varphi}{\sigma_{1, t}}\left(\omega_{t}+\left(1-\omega_{t}\right) \tau y_{t}\right)
$$

Similarly, in an equilibrium without shorting in asset 1 we have $\kappa_{1, t}=\sigma_{1, t} \widetilde{m}_{1, t}-\left(1-\omega_{t}\right) \eta$ if investor $R$ holds an interior position in asset 1 and $\kappa_{1, t}=\frac{\sigma_{1, t} \widetilde{m}_{1, t}}{1-\omega_{t}}-\eta$ otherwise.

The excess return to asset 2 is given by the conventional CAPM relationship

$$
\mu_{2, t}-r_{t}=[0,1] \sigma_{t} \sigma_{t}^{\prime} \vec{m}_{t}
$$

Equations (40) and (39) specialize to (25) and (24), respectively, when $\widetilde{m}_{1, t}=1$ and $\sigma_{1, t}=\sigma_{D}$. In this sense, Proposition 5 is a natural extension of Proposition 2, except that the Sharpe ratio in Proposition 5 pertains to a portfolio that invests one dollar in asset 1 and shorts $b_{t}$ units of asset 2 (so as to "hedge out" the exposure of the portfolio to the second Brownian shock).

As in Proposition 2, the excess return on asset 1 can be decomposed into a risk premium, a (wealth-weighted) belief distortion, and a component that reflects the impact of shorting costs. Specifically, equation 40 implies that in an equilibrium with active shorting, the expected return of stock 1 is

$$
\mu_{1, t}-r_{t}=\underbrace{b_{t}\left(\mu_{2, t}-r_{t}\right)+\widetilde{m}_{1, t} \sigma_{1, t}^{2}}_{\text {risk compensation }}-\underbrace{\left(1-\omega_{t}\right) \eta \sigma_{1, t}}_{\text {wealth-weighted optimism }}-\underbrace{\varphi\left(\omega_{t}+\left(1-\omega_{t}\right) \tau y_{t}\right)}_{\text {impact of shorting costs }} .
$$

All else equal, a higher level of $y_{t}$ lowers $\mu_{1, t}-r$ - consistent with the empirical finding that short interest negatively predicts returns - and higher values of the lending fee $\varphi$ lower 
equilibrium expected excess returns.

\subsection{A limiting economy with a small and a large stock}

The CAPM-style formulas provide equilibrium returns conditional on the equilibrium covariance matrix and the investor wealth shares. To fully solve the model, we consider a limiting, multi-stock economy, in which trees of type 1 are small compared to trees of type 2 and also the fraction of investors that pay attention to trees of type 1 is small. Since this section involves some detailed modeling assumptions, we relegate the full presentation to Appendix C. In the text we simply summarize the setup and the main findings.

Specifically, suppose that there are two types of trees, namely "small" trees (type-1 trees) and "large" trees (type-2 trees). Type-2 trees have dividends similar to the baseline model, namely $D_{2, t, s}=\phi_{2} \delta_{2} D_{2, t} e^{-\delta_{2}(t-s)}$, where $\phi_{2}>0, \delta_{2}>0$, and $D_{2, t}$ follows a geometric Brownian motion, $\frac{d D_{2, t}}{D_{2, t}}=\mu_{2, D} d t+\sigma_{2, D} d B_{2, t}$. Type-1 trees produce dividends

$$
D_{1, t, s}=\phi_{1} \delta_{1} D_{2, s} e^{-\delta_{1}(t-s)+\sigma_{1, D}\left(B_{1, t}-B_{1, s}\right)},
$$

with $\phi_{1}>0$ and $\delta_{1}>0$. With the above dividend specifications, the dividend ratio of type-1 to type- 2 trees is

$$
\frac{D_{1, t}}{D_{2, t}}=\frac{\int_{-\infty}^{t} D_{1, t, s} d s}{\int_{-\infty}^{t} D_{2, t, s} d s}=\frac{\phi_{1}}{\phi_{2}} \int_{-\infty}^{t} \frac{D_{2, s}}{D_{2, t}} \delta_{1} e^{-\delta_{1}(t-s)+\sigma_{1, D}\left(B_{1, t}-B_{1, s}\right)} d s,
$$

which is a stationary process.

The above assumptions imply that the dividend shares of type- 1 and type- 2 trees at an arbitrary time $t$ are stationary fractions of aggregate consumption $D_{1, t}+D_{2, t}$, while the dividends of the tree with which a fixed cohort $s$ is endowed upon entering the economy follow a geometric Brownian motion 25 Moreover, when type-1 trees are small compared

\footnotetext{
${ }^{25}$ Ito's Lemma implies that

$$
\frac{d D_{1, t, s}}{D_{1, t, s}}=\left(\frac{\sigma_{1, D}^{2}}{2}-\delta_{1}\right) d t+\sigma_{1, D} d B_{1, t} \text { and } \frac{d D_{2, t, s}}{D_{2, t, s}}=\left(\mu_{2, D}-\delta_{2}\right) d t+\sigma_{2, D} d B_{2, t} .
$$
}


to type-2 trees $\left(\frac{\phi_{1}}{\phi_{2}} \approx 0\right)$, aggregate consumption is approximately equal to the aggregate dividends of the large, type-2 trees, and therefore aggregate consumption follows a geometric Brownian motion. The implication is that the interest rate and the risk premium for type-2 trees both converge to constants as the ratio $\frac{\phi_{1}}{\phi_{2}} \rightarrow 0$ goes to zero.

In the baseline model, entry and exit of investors into the single stock market was tied to the arrival and departure of agents in the economy and was essentially exogenous. The extension to two risky-asset markets requires that we model the entry and exit into the market for stock 1. Specifically, we assume that investors of both types $(R$ and $I)$ gain and lose interest in stock 1 at the rate $\chi$ per unit of time $d t$. Phrased differently, a measure $\chi$ of investors becomes interested in market 1 per unit of time and a measure $\chi$ of investors loses interest for exogenous reasons. Of the arriving investors a fraction $\nu$ is of type $R$, as in the baseline model.

We go further and make an additional assumption that captures the shifts in investorbase composition that can accompany shifts in equilibrium. Specifically, we assume that investors incur a small, non-pecuniary, disutility flow $\varepsilon$ from paying attention to stock 1 . An investor of type $i \in(I, R)$ will consequently choose to keep staying in the market if and only if her expected discounted utility from remaining attentive to stock 1 is above the present value of the (small) disutility cost of attention. To anticipate the results, this participation cost will drive some type- $R$ agents out of the market when no-shorting equilibria prevail, capturing the notion that short sellers lose interest in stock 1 in such times.

For more detail, observe first that, as long as this disutility is small enough, it is irrelevant for investors of type $I$, since they always choose a strictly positive portfolio in stock 1 . For investors of type $R$, however, there are regions of $\omega_{t}$ where their optimal holding of stock 1 is zero. Even a small disutility, therefore, can lead them to exit the market. Formally, an investor of type $R$ finds it optimal to remain in the market for stock 1 if and only if

$$
V^{R}\left(\omega_{t}\right) \equiv \mathrm{E}_{t}\left[\max _{w_{1, u}^{R}} \int_{t}^{T} e^{-\rho(u-t)}\left(w_{1, u}^{R}\left(\mu_{u}-r_{u}+\lambda_{u}^{R}\right)-\frac{1}{2}\left(w_{1, u}^{R} \sigma_{u}\right)^{2}-\varepsilon\right) d u\right] \geq 0
$$

where $T$ is the stochastic time of exit from the market for stock 1 (be it endogenous or exogenous). Equation (44) uses the assumption of logarithmic preferences - along with the 
simplifying assumption that stocks 1 and 2 are independent — to express the net expected utility gain from continued presence in market 1 as the increase in investor $R$ 's logarithmic growth rate of wealth, $w_{1, u}^{R}\left(\mu_{u}-r_{u}+\lambda_{u}^{R}\right)-\frac{1}{2}\left(w_{1, u}^{R} \sigma_{u}\right)^{2}$, net of the flow disutility of presence in the market $\varepsilon$. The requirement that this net gain stay positive at all times implies that, for given equilibrium functions $\kappa\left(\omega_{t}\right)$ and $y\left(\omega_{t}\right)$, there is a critical boundary $\bar{\omega}$, typically lying in the region of $\omega_{t}$ where $w_{1, u}^{R}\left(\omega_{t}\right)=0$, that acts as a "reflecting barrier" for $\omega_{t}$. Specifically, if the process $\omega_{t}$ were to ever exceed $\bar{\omega}$, there would be enough exit to restore $\omega_{t}$ back to $\bar{\omega} \cdot{ }^{26}$

Some further technical assumptions on investor entry and exit are detailed in Appendix C. Here we simply state the main result, which provides an ordinary differential equation (ODE) for the price-dividend ratio. For simplicity, we assume that the Brownian motions $B_{1, t}$ and $B_{2, t}$ are independent.

Proposition 6 Using the expressions for $w_{t}^{i}, \kappa_{1, t}($ with $b=0)$, and $y_{t}$ from Proposition 5 , the wealth share $\omega_{t}$ follows the diffusion process

$$
d \omega_{t}=\mu_{t}^{R} d t+\sigma_{t}^{R} d B_{1, t}-d F_{t}
$$

where $F_{t}$ is an increasing (singular) process that reflects $\omega_{t}$ to remain below the value $\bar{\omega}$ that makes equation (44) hold as an equality and $\mu_{t}^{R}$ and $\sigma_{t}^{R}$ are given by

$$
\begin{aligned}
& \mu_{t}^{R}=\omega_{t}\left(\left(w_{1, t}^{R}-\tilde{m}\right) \sigma_{1, t}\left(\kappa_{t}-\sigma_{1, t} \widetilde{m}\right)+w_{1, t}^{R} \varphi+\frac{y_{t} \widetilde{m}}{1-y_{t}} \varphi(1-\tau)\right)+\chi\left(\nu-\omega_{t}\right), \\
& \sigma_{t}^{R}=\omega_{t}\left(w_{1, t}^{R}-\widetilde{m}\right) \sigma_{1, t},
\end{aligned}
$$

where $\sigma_{1, t}=\frac{p^{\prime}\left(\omega_{t}\right)}{p\left(\omega_{t}\right)} \sigma_{t}^{R}+\sigma_{1, D}$ is the volatility of stock 1 and the price-dividend ratio $p_{t}=p\left(\omega_{t}\right)$ solves the ordinary differential equation

$$
\frac{1}{2} \frac{\partial^{2} p}{\partial \omega_{t}^{2}}\left(\sigma_{t}^{R}\right)^{2}+\frac{\partial p}{\partial \omega_{t}}\left(\mu_{t}^{R}+\left(\sigma_{1, D}-\kappa_{1, t}\right) \sigma_{t}^{R}\right)-p\left(r+\delta_{1}+\kappa_{1, t} \sigma_{1, D}\right)+1=0
$$

in the region $0 \leq \omega_{t} \leq \bar{\omega}$.

Remark 2 Since there are multiple equilibrium values for $w_{t}^{i}, \kappa_{1, t}$, and $y_{t}$ in Proposition

\footnotetext{
${ }^{26}$ This behavior is reminiscent of models of industry equilibrium with endogenous entry and exit (e.g., Leahy (1993), Baldursson and Karatzas (1996).)
} 
6. there are a continuum of solutions for $p(\cdot)$ and $\bar{\omega}$, depending on the equilibria on which agents coordinate at each value of $\omega_{t}$.

The expressions for $\mu_{t}^{i}$ and $\sigma_{t}^{i}$ in Proposition 6 coincide with (18) and (17) when $\widetilde{m}=1$, $\chi=\pi$, and $\sigma_{1, t}=\sigma_{D}{ }^{27}$ Moreover, with the dividend growths of stocks 1 and 2 being independent, so are their stock-price processes (in the limit where stock 1 becomes small) and the expressions for $y_{t}, w_{1, t}^{i}$, and $\kappa_{1, t}$ in Proposition 6 (when $\widetilde{m}=1$ and $\sigma_{1, t}=\sigma_{D}$ ) coincide with the respective expressions in the baseline model. Finally, if $\varepsilon=0$, then $\bar{\omega}=1$, as in the baseline model. In short, if one dropped the goods-market clearing requirement from the baseline model, the resulting expression for the price-to-dividend ratio would be given by (48) (with $\widetilde{m}=1$ and $\varepsilon=0$ ).

The main complications with solving (48) are that a) it is a non-linear ODE ${ }^{28}$ and b) if $\varepsilon>0$, this ODE is to be solved over a domain of values of $\omega_{t}$ so that (44) holds, together with the boundary $\bar{\omega}$ implied by Equation (44). The appendix describes an iterative numerical procedure to solve this problem conditional on the equilibrium that investors coordinate on at each value of $\omega_{t}$.

To illustrate the results, Figure 11 presents the solution for the price-dividend ratio. We are interested in situations where the disagreement is large $(\eta=0.9)$, and the speed of investor churn in market 1 is quite large $(\chi=2)$, to capture short-termism. The idiosyncratic dividend volatility is not too large, $\sigma_{1, D}=7 \%$, and the shorting fee is at the high levels that one encounters for stocks that are "on special" $(\varphi=5.7 \%)$. A proportion $\nu=0.7$ of new investors are of type $R$. In equilibrium, this value of $\nu$ ensures that the endogenous exit decision is meaningful, that is, under any equilibrium there would a possibility that $\omega_{t}$ "spends time" in a region where a zero holding of asset 1 is optimal for investor $R$. Finally, we assume that the sum of interest rate and depreciation $r+\delta_{1}$ for stock 1 is 0.1 . We choose a value of $\tau=0.8$ based on the industry practice of rebating about $80 \%$ to the mutual funds or ETFs that provide their shares for lending ${ }^{29}$ Finally, for the disutility $\varepsilon$ we intentionally

\footnotetext{
${ }^{27}$ To see this, substitute the expression for the equilibrium interest rate 26 to 18 .

${ }^{28}$ The reason why (48) is non-linear is that $\mu_{t}^{i}$ and $\sigma_{t}^{i}$ depend on $\sigma_{1, t}$, which in turn depends on $p\left(\omega_{t}\right)$ and $p^{\prime}\left(\omega_{t}\right)$.

${ }^{29}$ Source: "Unlocking the potential of your portfolios: iShares Security Lending." Blackrock, 2021. Available at https://www.ishares.com.
} 


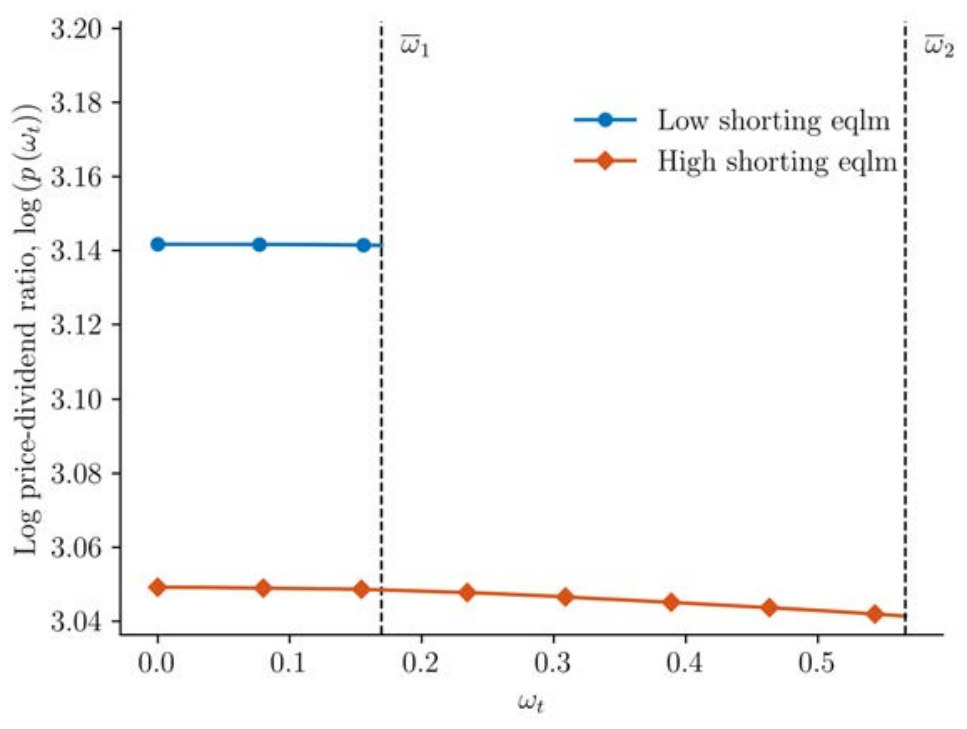

Figure 11: The price-dividend ratio in the equilibria involving the highest and the lowest extent of shorting.

choose a very small amount (2.5 basis points).

Figure 11 shows the price-dividend ratio under two different assumptions on the equilibrium that investors coordinate on (when multiple equilibria are possible). Specifically, the line "low shorting" assumes that investors always coordinate on the equilibrium with zero shorting, if it exists. By contrast, the line "high shorting" assumes that investors always coordinate on the equilibrium with the highest possible shorting. Note that both lines extend only until the levels $\bar{\omega}_{1}$ and $\bar{\omega}_{2}$, respectively, which are the levels of $\omega_{t}$ at which $R$ investors exit in the two equilibria.

There are several noteworthy features of Figure 11. First, the price-dividend ratio for the low shorting equilibrium is higher than the price-dividend ratio for the high shorting equilibrium. This may seem counterintuitive in light of the fact that the high shorting equilibrium implies a lower Sharpe ratio for a fixed $\omega_{t}$. The reason is that in the low shorting equilibrium many $R$ investors do not maintain a presence in the market for stock 1. In that equilibrium shorting occurs only for low values of $\omega_{t}$ (below 0.3 in this numerical example), which realize infrequently. Consequently, a significant fraction of $R$ investors leave the market, meaning that the typical steady-state values of $\omega_{t}$ are low. The few remaining $R$ investors maintain a presence in the hope that, over the course of their stay in market 1 , 
the process for $\omega_{t}$ may drift even lower, thus activating shorting - a low probability event in this equilibrium. Since the (stochastic) steady-state wealth share of rational investors, $\omega_{t}$, is quite low, so is the Sharpe ratio, and thus the price-dividend ratio is high.

By contrast, the high shorting equilibrium attracts more $R$ investors, who rationally anticipate that they will be actively shorting (with high probability) over the course of their stay in the market for stock 1 . Therefore, the typical values of $\omega_{t}$ are higher, the Sharpe ratio is higher, and the price-dividend ratio is lower than in the low-shorting equilibrium.

We also note that our assumption of a high $\chi$ ("short-termism") is reflected in the fact that the curves for the price-dividend ratio are essentially flat lines, since the process $\omega_{t}$ mean-reverts quickly to its stochastic steady state under either equilibrium. Because of this feature, the price-dividend ratio in either equilibrium is roughly equal to its steady-state value irrespective of the current value of $\omega_{t}$.

In terms of quantities, Figure 11 implies that an unanticipated shift in equilibrium (from the "high shorting" to the "low shorting") will make the price-dividend ratio jump upward by about $10 \%$.

\section{Conclusion}

In this paper we propose a theory for why shorting can be fickle and subject to sudden reversals, not attributable to a change in the fundamentals or the stock lending technology. To illustrate the notion of sudden reversals, we use a time-honored device in economic theory, namely the existence of multiple equilibria - a modeling device to illustrate the feedback effects between the spot market and the lending market.

In the model, shorting can exhibit "run-type" patterns. An event that prompts some short sellers to abandon their short positions can ignite a self-propagating cycle: Less shorting also implies less lending income for investors with long positions, who now need to be compensated with a higher Sharpe ratio, which in turn further prompts short sellers to abandon their strategies. (Going in the opposite direction, a high level of shorting activity further subsidizes long positions, thus lowering the equilibrium Sharpe ratio and attracting further short selling). Thus, for the same fundamentals, there can be multiple equilibria - 
a manifestation of the self-reinforcing nature of shorting decisions.

The paper can consequently provide an explanation of how a mutually-shared shift in perception about the market environment can spiral quickly across many shorting strategies. The model can also help explain a simultaneous decline in short selling and rise in the price of a stock (such as the one that we document in our empirical analysis) even when neither lending fees, nor fundamentals, nor the supply curve for lendable shares change. At first sight, it would appear that a rise in the stock price (absent a change in fundamentals or lending fees) should attract, rather than repel short sellers. We show, though, that the likelihood of a switch to a no-shorting equilibrium can drive the incentive of participating in the market low enough to prompt would-be short sellers to "abandon" the asset to the optimists.

While motivated by recent events, the analysis of the paper has broader implications for the empirical relationship between shorting and stock returns. One aspect we wish to highlight is that this model helps explain why shifts in the supply of lendable shares do not have a clear impact on the Sharpe ratio. 


\section{References}

Allen, F., M. Haas, E. Nowak, M. Pirovano, and A. Tengulov (2021). Squeezing shorts through social media platforms. History of Finance eJournal.

Asquith, P., P. A. Pathak, and J. R. Ritter (2005). Short interest, institutional ownership, and stock returns. Journal of Financial Economics 78(2), 243-276.

Atmaz, A., S. Basak, and F. Ruan (2020). Dynamic equilibrium with costly short-selling and lending market. Working paper.

Baldursson, F. M. and I. Karatzas (1996). Irreversible investment and industry equilibrium. Finance and Stochastics 1(1), 69-89.

Banerjee, S. and J. J. Graveline (2013). The cost of short-selling liquid securities. Journal of Finance 68(2), 637-664.

Baumgartner, J., S. Zannettou, B. Keegan, M. Squire, and J. Blackburn (2020). The pushshift reddit dataset.

Beneish, M. D., C. M. Lee, and D. C. Nichols (2015). In short supply: Short-sellers and stock returns. Journal of Accounting and Economics 60(2-3), 33-57.

Benhabib, J. and R. Farmer (1999). Indeterminacy and sunspots in macroeconomics. In J. B. Taylor and M. Woodford (Eds.), Handbook of Macroeconomics, Volume 1, Part A, Chapter 06, pp. 387-448.

Biais, B., J. Hombert, and P.-O. Weill (2021). Incentive constrained risk sharing, segmentation, and asset pricing. American Economic Review, forthcoming.

Blanchard, O. J. (1985). Debt, deficits, and finite horizons. Journal of Political Economy 93(2), 223-247.

Blocher, J., A. V. Reed, and E. D. Van Wesep (2013). Connecting two markets: An equilibrium framework for shorts, longs, and stock loans. Journal of Financial Economics 108(2), $302-322$.

Boehmer, E., C. M. Jones, and X. Zhang (2008). Which shorts are informed? Journal of Finance 63(2), 491-527.

Boehmer, E., C. M. Jones, X. Zhang, and X. Zhang (2020). Tracking retail investor activity. Journal of Finance, forthcoming.

Cohen, L., K. B. Diether, and C. J. Malloy (2007). Supply and demand shifts in the shorting market. Journal of Finance 62(5), 2061-2096.

Dechow, P. M., A. P. Hutton, L. Meulbroek, and R. G. Sloan (2001). Short-sellers, fundamental analysis, and stock returns. Journal of Financial Economics 61(1), 77-106. 
Desai, H., K. Ramesh, S. R. Thiagarajan, and B. V. Balachandran (2002). An investigation of the informational role of short interest in the Nasdaq market. Journal of Finance 57(5), $2263-2287$.

Detemple, J. and S. Murthy (1997). Equilibrium asset prices and no-arbitrage with portfolio constraints. The Review of Financial Studies 10(4), 1133-1174.

Diamond, D. W. and R. E. Verrecchia (1987). Constraints on short-selling and asset price adjustment to private information. Journal of Financial Economics 18(2), 277-311.

Diether, K. B., K.-H. Lee, and I. M. Werner (2009). Short-sale strategies and return predictability. The Review of Financial Studies 22(2), 575-607.

Drechsler, I. and Q. F. Drechsler (2014). The shorting premium and asset pricing anomalies. Technical report, National Bureau of Economic Research.

Duffie, D., N. Gârleanu, and L. H. Pedersen (2002). Securities lending, shorting, and pricing. Journal of Financial Economics 66(2-3), 307-339.

Duong, T. X., Z. R. Huszár, R. S. K. Tan, and W. Zhang (2017, 01). The Information Value of Stock Lending Fees: Are Lenders Price Takers? Review of Finance 21 (6), 2353-2377.

D'Avolio, G. (2002). The market for borrowing stock. Journal of Financial Economics 66 (2), $271-306$.

Farmer, R. and J.-P. Bouchaud (2020, December). Self-fulfilling prophecies, quasi nonergodicity \& wealth inequality. Working Paper 28261, National Bureau of Economic Research.

Fostel, A. and J. Geanakoplos (2008). Leverage cycles and the anxious economy. American Economic Review 98(4), 1211-44.

Gârleanu, N., L. Kogan, and S. Panageas (2012). Displacement risk and asset returns. Journal of Financial Economics 105(3), 491-510.

Gârleanu, N. and S. Panageas (2015). Young, old, conservative, and bold: The implications of heterogeneity and finite lives for asset pricing. Journal of Political Economy 123(3), 670-685.

Gârleanu, N. and S. Panageas (2020). Heterogeneity and asset prices: A different approach. Technical report, National Bureau of Economic Research.

Gârleanu, N. and S. Panageas (2021). What to expect when everyone is expecting: Selffulfilling expectations and asset-pricing puzzles. Journal of Financial Economics 140(1), $54-73$.

Geczy, C. C., D. K. Musto, and A. V. Reed (2002). Stocks are special too: an analysis of the equity lending market. Journal of Financial Economics 66(2), 241-269. Limits on Arbitrage. 
Harrison, J. M. and D. M. Kreps (1978). Speculative investor behavior in a stock market with heterogeneous expectations. Quarterly Journal of Economics 92(2), 323-336.

Hong, H. and J. C. Stein (2003). Differences of opinion, short-sales constraints, and market crashes. The Review of Financial Studies 16(2), 487-525.

Jones, C. M. and O. A. Lamont (2002). Short-sale constraints and stock returns. Journal of Financial Economics 66(2-3), 207-239.

Kaplan, S. N., T. J. Moskowitz, and B. A. Sensoy (2013). The effects of stock lending on security prices: An experiment. Journal of Finance 68(5), 1891-1936.

Karatzas, I. and S. Shreve (2012). Brownian motion and stochastic calculus, Volume 113. Springer Science \& Business Media.

Khorrami, P. and F. Mendo (2021). Rational sentiments and financial frictions. Working paper.

Khorrami, P. and A. Zentefis (2020). Arbitrage and beliefs. Working paper.

Kyle, A. S. and W. Xiong (2001). Contagion as a wealth effect. Journal of Finance 56(4), 1401-1440.

Lamont, O. A. (2012). Go down fighting: Short sellers vs. firms. The Review of Asset Pricing Studies 2(1), 1-30.

Lamont, O. A. and J. C. Stein (2004). Aggregate short interest and market valuations. American Economic Review 94(2), 29-32.

Leahy, J. V. (1993). Investment in competitive equilibrium: The optimality of myopic behavior. Quarterly Journal of Economics 108(4), 1105-1133.

Michel, J.-B., Y. K. Shen, A. P. Aiden, A. Veres, M. K. Gray, Google Books Team, J. P. Pickett, D. Hoiberg, D. Clancy, P. Norvig, J. Orwant, S. Pinker, M. A. Nowak, and E. L. Aiden (2011). Quantitative analysis of culture using millions of digitized books. Science 331(6014), 176-182.

Miller, E. M. (1977). Risk, uncertainty, and divergence of opinion. Journal of Finance 32(4), 1151-1168.

Panageas, S. (2020). The implications of heterogeneity and inequality for asset pricing. Technical report, National Bureau of Economic Research.

Pedersen, L. H. (2021). Game on: Social networks and markets. Technical report, Copenhagen Business School.

Porras Prado, M., P. A. C. Saffi, and J. Sturgess (2016, 07). Ownership Structure, Limits to Arbitrage, and Stock Returns: Evidence from Equity Lending Markets. The Review of Financial Studies 29(12), 3211-3244. 
Rapach, D. E., M. C. Ringgenberg, and G. Zhou (2016). Short interest and aggregate stock returns. Journal of Financial Economics 121(1), 46-65.

Scheinkman, J. A. and W. Xiong (2003). Overconfidence and speculative bubbles. Journal of Political Economy 111(6), 1183-1220.

Senchack, A. J. and L. T. Starks (1993). Short-sale restrictions and market reaction to shortinterest announcements. Journal of Financial and Quantitative Analysis 28(2), 177-194.

Seneca, J. J. (1967). Short interest: bearish or bullish? Journal of Finance 22(1), 67-70.

Shleifer, A. and R. W. Vishny (1997). The limits of arbitrage. Journal of Finance 52(1), $35-55$.

Simsek, A. (2013). Belief disagreements and collateral constraints. Econometrica 81(1), $1-53$.

Vayanos, D. and P.-O. Weill (2008). A search-based theory of the on-the-run phenomenon. Journal of Finance 63(3), 1361-1398.

Zentefis, A. (2018). Self-fulfilling asset prices. Working paper. 


\section{For Online Publication - Appendix}

\section{A The Determination of the Lending Fee}

In the text we assume a "flat" supply curve for lending shares. That is, we assume $f_{t}=$ $l\left(y_{t}\right)=\varphi$. We provide here the simplest model that supports this assumption. We also discuss how to extend the model to allow for an increasing $l(\cdot)$.

All interactions considered in this section happen anew every period, where the length of the period is idealized to be " $d t$," that is, infinitesimal. (We could formalize this assumption by considering a discrete-time model where the length $\Delta$ of a period is taken to go to zero, and focusing on the limit of resultant equilibria.)

We start by considering the long investors, who wish to lend their shares. Each investor lends all her shares to any one of a competitive fringe of risk-neutral "lender's dealers" in exchange for an income stream that is proportional to the dollar value of shares the investor lends. This income stream is determined as follows. In equilibrium, each broker receives a fee $f_{l}$ per dollar of shares it lends out, which constitute only a proportion $y_{t}$ of the shares it borrows from investors. (We omit time subscripts from now on.) Therefore, competition between the brokers drives the income stream of investors to $y f_{l}$ per dollar of shares they lend.

At the other end of the lending transaction, desirous short sellers interact with a competitive set of "borrower's brokers." Specifically, for every borrowing fee $f_{b}$ the would-be short sellers provide the dollar amount that they would like to short, and the brokers take the value $f_{b}$ as a given when they attempt to fill the investor's borrowing orders.

All of the frictions in this model pertain to the interaction between lender's dealers and borrower's brokers. Specifically, to initiate a stock loan the representative broker must pay a cost $\xi$ per dollar value of share "located" with a dealer, per unit of time. This cost is construed as labor cost that compensates brokers for their disutility of labor.

The interaction between the broker and the dealer takes the form of bilateral Nash bargaining in which the broker has bargaining power $1 /(1+z)$ for a parameter $z \in(0, \infty)$. Given our assumption that all interactions (between investors and brokers or dealers and between brokers and dealers) happen anew every period, the outside option for both brokers and dealers is the failure to transact during the period. This means that the gains from trade to the dealer equal the lending fee $f_{l}$, while to the broker the borrowing fee net of the lending one $f_{b}-f_{l}$ - the searching and matching cost $\xi$ has been sunk at this point. The total gains from trade equal $f_{b}$, the foregone revenue from the would-be short seller. Given the bargaining protocol, it follows that

$$
f_{l}=\frac{z}{1+z}\left(f_{l}+f_{b}-f_{l}\right)=\frac{z}{1+z} f_{b} .
$$

Since brokers are competitive, they break even on net, meaning that

$$
f_{b}=f_{l}+\xi
$$


so that

$$
\begin{aligned}
f_{l} & =z \xi \\
f_{b} & =(1+z) \xi .
\end{aligned}
$$

To keep the model transparent and tractable, assume that all brokers are members of the representative household, and therefore the fees that compensate them for their effort are rebated to each households as an income stream proportional to the household's wealth and independent of the composition of the household's portfolio.

Setting $\beta=(1+z) \xi$ and $\tau=z /(1+z)$, this extended model is equivalent to the model we assumed in the text. To generalize to upward-sloping supply curves, one would simply assume an increasing cost $\xi(y)$.

\section{B Multiple Agent Types}

We illustrate here that the multiplicity of equilibria may expand with the number of agent types. In particular, adding a third group of agents can result in a third equilibrium featuring non-zero shorting; such a model may admit, in fact, up to five equilibria.

Specifically, let us assume a third group of investors characterized by beliefs that are summarized by the quantity $\eta^{P}$. We think of these investors as pessimists, which implies $\eta^{P}<0$. The intuition we wish to capture is that, in addition to the "high-shorting" and "medium-shorting" equilibria in the base-line model, low-shorting equilibria may exist in which investor $R$ is inactive, while investor $P$ shorts actively.

To make the point theoretically, one may argue by continuity. Specifically, consider the zero-shorting equilibrium in the baseline model, and perturb the setting by adding a small mass of sufficiently pessimistic investors $\left(\left|\eta^{P}\right|\right.$ large enough). These investors will want to short, but will not be sufficiently numerous to move the Sharpe ratio or lending income to a point where investors $R$ and $I$ are no longer in equilibrium.

It is helpful to write down the equilibrium conditions in the augmented model - both to allow for a formal argument and in the interest of a numerical illustration. We repeat the analysis in the text - letting $\omega^{P}$ denote the wealth share of agents $P$ - to obtain the market clearing condition

$$
\begin{aligned}
1= & \frac{1}{\sigma_{D}}\left[\omega^{P}\left(\kappa+\eta^{P}+\frac{\varphi}{\sigma_{D}}\right) 1_{\left\{\kappa+\eta^{P}+\frac{\varphi}{\sigma_{D}}<0\right\}}+\omega^{R}\left(\kappa+\frac{\varphi}{\sigma_{D}}\right) 1_{\left\{\kappa+\frac{\varphi}{\sigma_{D}}<0\right\}}+\right. \\
& \left.\omega^{I}\left(\kappa+\eta^{I}+\frac{\varphi}{\sigma_{D}} \tau y\right)\right]
\end{aligned}
$$

where the left-hand side is the proportion of aggregate wealth represented by the supply of the stock, while the right-hand side equals the proportion of aggregate wealth invested in the stock. We restricted attention to cases in which $R$ agents do not take a long position in the stock. 
We solve for the Sharpe ratio $\kappa$ :

$$
\kappa=\sigma_{D}-\left(\omega^{P} \eta^{P}+\omega^{I} \eta^{I}\right)-\frac{\varphi}{\sigma_{D}}\left(\omega^{P}+\omega^{R}+\omega^{I} \tau y\right)
$$

if $\kappa+\varphi / \sigma_{D}<0$, respectively

$$
\kappa=\frac{\sigma_{D}}{\omega^{P}+\omega^{I}}-\frac{\omega^{P} \eta^{P}+\omega^{I} \eta^{I}}{\omega^{P}+\omega^{I}}-\frac{\varphi}{\sigma_{D}} \frac{\omega^{P}+\omega^{I} \tau y}{\omega^{P}+\omega^{I}}
$$

if $\kappa+\varphi / \sigma_{D} \geq 0>\kappa+\eta^{P}+\varphi / \sigma_{D}$.

The other equilibrium condition concerns the determination of the value of $y$ :

$$
y=-\frac{\omega^{P}\left(\kappa+\eta^{P}+\frac{\varphi}{\sigma_{D}}\right) 1_{\left\{\kappa+\eta^{P}+\frac{\varphi}{\sigma_{D}}<0\right\}}+\omega^{R}\left(\kappa+\frac{\varphi}{\sigma_{D}}\right) 1_{\left\{\kappa+\frac{\varphi}{\sigma_{D}}<0\right\}}}{\omega^{I}\left(\kappa+\eta^{I}+\frac{\varphi}{\sigma_{D}} \tau y\right)} .
$$

Depending on whether $\kappa$ is determined according to $(\mathrm{B} .2)$ or $(\mathrm{B} .3)$ we obtain a different quadratic equation. For appropriate parameter choices all but one combinations are possible in terms of how many solutions in the interval $(0,1)$ each of them admits. We are particularly interested in situations in which (B.2) applies and results in two admissible solutions, in addition to which at least one solution obtains when $(\mathrm{B} .3)$ applies.

We illustrate such outcomes in Figure 12. The two panels differ in terms of parameters, but depict the same objects. Specifically, the x-axis records candidate values of short interest $y$ that agents anticipate. Agents form demands taking such a value $y$ and a Sharpe ratio $\kappa$ as given, and clearing in the asset market determines the Sharpe ratio. With the Sharpe ratio now specified for each candidate $y$, we can compute the actual resulting short interest - the value of the right-hand side of equation (B.4). This quantity is recorded on the y-axis. An equilibrium requires that the $\mathrm{x}$ and $\mathrm{y}$ coordinates are equal.

The line " $R$ and $P$ short" plots $y$ as if both $R$ and $P$ shorted, that is, their portfolio weights are calculated by adding the return $\varphi$ to their perceived intrinsic expected return from the asset; in that case, the Sharpe ratio is given by $(\bar{B} .2)$. The line "Only $P$ shorts" is produced similarly, except that the demand of agent $R$ is set to zero; equation (B.3) applies. The actual resulting short interest is depicted by the thick continuous line, labeled "Actual response." Finally, the line "Diagonal" depicts the equilibrium condition. Equilibria are therefore represented by points of intersection between the two continuous lines. The left panel presents a situation in which four equilibria with positive amounts of shorting and one with zero shorting obtain. The right panel presents a situation with three equilibria, all of which feature positive short interest.

We also flesh out the theoretical argument for the existence of a third equilibrium when $\omega^{P}$ is close to zero and two equilibria with $y>0$ exist with $\omega^{P}=0$ - i.e., the baseline model. By assumption, with $\omega^{P}=0$ and $y=0$ equation (B.3) applies and $\kappa+\frac{\varphi}{\sigma_{D}}>0$. 

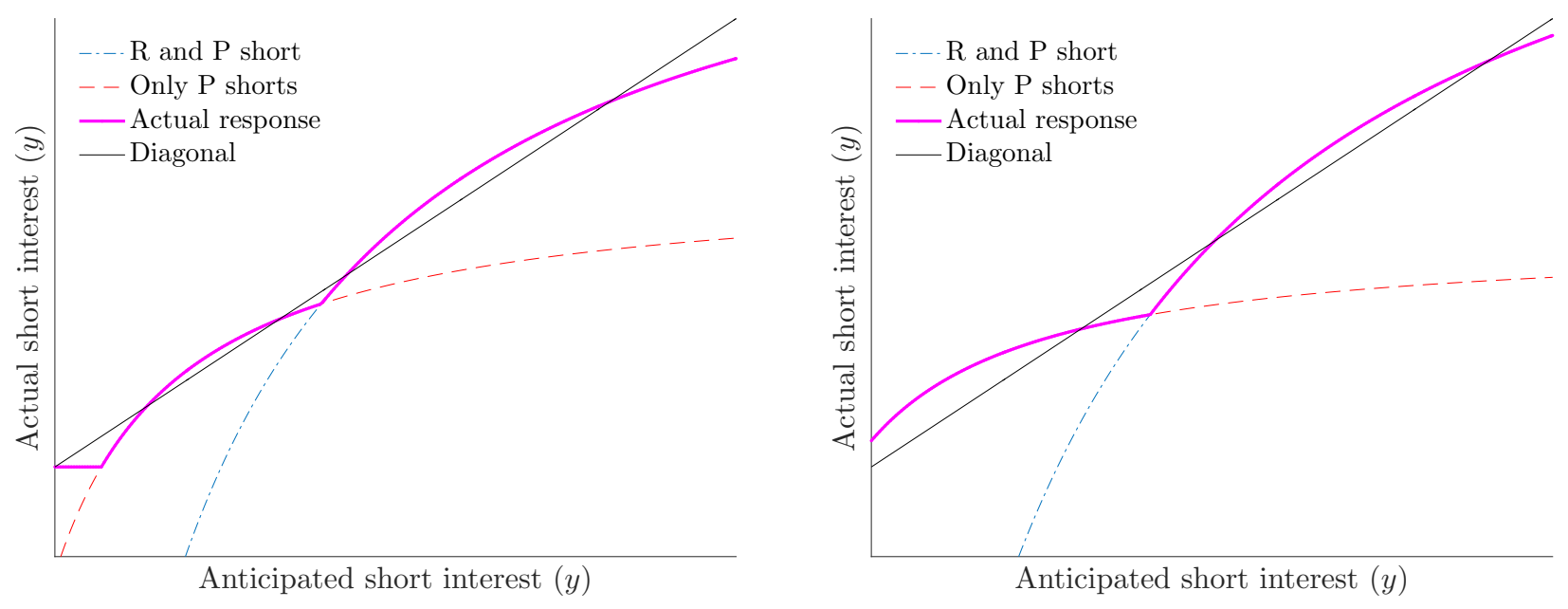

Figure 12: The figure plots, in each panel, four lines pertaining to the model extension developed in this section. Equilibria are characterized by the satisfaction of equation (B.4), whose right-hand side is represented here by the line "Actual response" and the left-hand side by the line "Diagonal." Further details are provided in the text.

Choosing $\eta^{P}$ so that $\kappa+\eta^{P}+\frac{\varphi}{\sigma_{D}}<0$, we wish to conclude that equations

$$
y=-\frac{\omega^{P}\left(\kappa+\eta^{P}+\frac{\varphi}{\sigma_{D}}\right)}{\omega^{I}\left(\kappa+\eta^{I}+\frac{\varphi}{\sigma_{D}} \tau y\right)}
$$

and (B.3) admit a solution that satisfies $\kappa+\frac{\varphi}{\sigma_{D}}>0$ even for $\omega^{P}>0$, at least when it is small enough. For simplicity, we keep $\omega^{I}$ constant as we increase $\omega^{P}$ from zero. Plugging B.3 in B.5 we obtain a quadratic that can be written as

$$
y=\frac{\omega^{P}}{\omega^{I}} \frac{\left(\eta^{I}-\eta^{P}\right) \omega^{I}-\frac{\varphi}{\sigma_{D}} \omega^{I} \tau(1-y)-\sigma_{D}}{\left(\eta^{I}-\eta^{P}\right) \omega^{P}-\frac{\varphi}{\sigma_{D}} \omega^{P} \tau(1-y)+\sigma_{D}} \equiv H\left(\omega^{P}, y\right) .
$$

Our choice of $\eta^{P}$ is such that the numerator of the second fraction on the right-hand side is positive at $y=0$, which implies $\frac{\partial H}{\partial \omega^{P}}>0$ evaluated at $\omega^{P}=0$, as well as $\frac{\partial H}{\partial y}=0$ at $\omega^{P}=0$. We therefore have

$$
\frac{d y}{d \omega^{P}}=\left(1-\frac{\partial H}{\partial y}\right)^{-1} \frac{\partial H}{\partial \omega^{P}}>0
$$

confirming that an equilibrium with positive short interest exists for small $\omega^{P}$. (The condition $\kappa+\frac{\varphi}{\sigma_{D}}>0$ is satisfied by continuity.) 


\section{The Price-Dividend Ratio of a Small Stock}

This section provides the details of the entry-and-exit process for the model of Section 5.2 and proves Proposition 6 .

The entry and exit into market 1 happens either for endogenous or exogenous reasons. By "endogenous" we mean that investors conduct a cost-benefit analysis before deciding whether to keep paying attention to the market for stock 1. In addition to this optimizing choice, we assume that investors enter and exit the market for exogenous reasons. This exogenous flux of investors is modeled with the sole purpose of making the model solution more tractable and transparent.

Specifically, recalling that $W_{t}^{i}$ denotes the (aggregate) wealth of type- $i$ investors that participate in market 1, we assume

$$
d W_{t}^{i}=d W_{t}^{i, \text { part }}+\chi\left(\nu^{i}\left(W_{t}^{I}+W_{t}^{R}\right)-W_{t}^{i}\right) d t-1_{i=R} \times \frac{W_{t}^{I}+W_{t}^{R}}{1-\omega_{t}} d F_{t}+\omega_{t}^{i}\left(d L_{t}-d N_{t}\right),
$$

where $d W_{t}^{i \text {,part }}$ is the wealth growth of an investor of type $i \in\{I, R)$ who is already participating in the market for stock 130 The term $\chi\left(\nu_{i}\left(W_{t}^{I}+W_{t}^{R}\right)-W_{t}^{i}\right) d t$ reflects entirely exogenous, non-optimizing entry, which happens at some rate $\chi$.

As in the baseline model, we assume that this exogenous entry-and-exit process affects the composition, but not the sum, of $W_{t}^{I}+W_{t}^{R}$, since

$$
\sum_{i \in\{I, R\}} \chi\left(\nu^{i}\left(W_{t}^{I}+W_{t}^{R}\right)-W_{t}^{i}\right)=0
$$

The term $-1_{i=R} \times \frac{W_{t}^{I}+W_{t}^{R}}{1-\omega_{t}} d F_{t}$ captures the endogenous exit of $R$ investors. As in the text, the (singular) process $d F_{t}$ is constructed so that $\omega_{t}$ stays below the critical value of $\omega_{t}$, which ensures that (44) holds.

Mostly for technical tractability reasons, we assume another source of exogenous entry and exit, which is reflected in the term $\omega_{t}^{i}\left(d L_{t}-d N_{t}\right)$ on the right-hand side of (C.1). This entry and exit process leaves the composition of wealth in the market (between $R$ and $I$ investors) unaffected, but ensures that the wealth of the investors who pay attention to the market "1" stays proportional to the "size" of market 1. Specifically, we define $d L_{t}$ and $d N_{t}$ as the two singular, increasing processes that "control" $W_{t}^{I}+W_{t}^{R}$ so that the ratio of stock market capitalization of asset 1 to the total wealth of investors participating in market $1, \widetilde{m}_{t}=\frac{M_{1, t}}{W_{t}^{I}+W_{t}^{R}}$, stays constant across time $\left(\widetilde{m}_{t}=\widetilde{m}\right){ }^{31}$ Because $\left(d L_{t}-d N_{t}\right)$ is multiplied by $\omega_{t}^{i}$, this exogenous entry-and-exit process does not impact the composition of wealth between $R$ and $I$ investors. The purpose of this exogenous entry-and-exit term

${ }^{30}$ For completeness, $d W_{t}^{i, \text { part }}=W_{t}^{i, \text { part }} \mu_{W}^{i} d t+W_{t}^{i, \text { part }} \vec{\sigma}_{W}^{i} d W_{t}$, where

$$
\mu_{W}^{i}=r_{t}+\pi+n_{t}+\vec{w}_{t, s}^{i}\left(\vec{\mu}_{t}-r_{t} 1_{\{2 \times 1)}+\lambda_{t, s}^{i}\left[\begin{array}{l}
1 \\
0
\end{array}\right]\right)-\frac{c_{t, s}^{i}}{W_{t, s}^{i}}
$$

for any $s \leq t$ and $\vec{\sigma}_{W}^{i}=\vec{w}^{i \prime} \sigma_{t}$.

${ }^{31}$ These processes can be uniquely constructed from the running maximum and minimum of the difference between $\left(W_{t}^{R}+W_{t}^{I}\right)-M_{1, t}$. For details see Karatzas and Shreve $(2012$, p. 210) on the Skorohod equation. 
is transparency and tractability: By ensuring a constant $\widetilde{m}_{t}$, if there were no differences of opinion $(\eta=0)$, the excess return, the price-dividend ratio, and the volatility of stock 1 would all be constant. Thus, we can eliminate a state variable from the problem, namely the ratio of market capitalization to the total wealth of investors in market 1). Economically, this means that we can abstract from the economic effects of limited participation (that have been studied extensively in the literature) and isolate the impact of shorting frictions. It is also worth highlighting that the term $\omega_{t}^{i}\left(d L_{t}-d N_{t}\right)$ would endogenously approach zero as $\delta_{1}$ and $\chi$ approach infinity ${ }^{32}$ Thus, our computations would be approximately valid, if we eliminated the term $\omega_{t}^{i}\left(d L_{t}-d N_{t}\right)$, as long as the analysis focuses on cases where investors are short-termist ( $\chi$ is large) and the ratio of the dividends of a typical tree 1 to tree 2 mean reverts fast.

Having described the entry and exit of investors into the market for stock 1, we now proceed to derive the differential equation in Proposition 6. Using the market clearing condition $\sum_{i \in\{I, R\}} \omega_{t}^{i} w_{t}^{i, 1}=\widetilde{m}$, and applying Ito's Lemma to $\omega_{t}^{i}=\frac{W_{t}^{i}}{W_{t}^{I}+W_{t}^{R}}$ leads to

$$
d \omega_{t}^{i}=\mu_{t}^{i} d t+\sigma_{t}^{i} d B_{1, t}
$$

where

$$
\begin{aligned}
\mu_{t}^{i} & =\omega_{t}^{i}\left[\left(w_{1, t}^{i}-\widetilde{m}\right) \sigma_{1, t}\left(\kappa_{t}-\sigma_{1, t} \tilde{m}\right)+w_{1, t}^{i} f_{t}+\widetilde{n}_{t}\right]+\chi\left(\nu_{t}^{i}-\omega_{t}^{i}\right), \\
\sigma_{t}^{i} & =\omega_{t}^{i}\left(w_{1, t}^{i}-\widetilde{m}\right) \sigma_{1, t},
\end{aligned}
$$

and 33

$$
\widetilde{n}_{t} \equiv-\sum_{i \in\{I, R\}} w_{1, t}^{i} \omega_{t}^{i} \lambda_{t}^{i}=\frac{y_{t} \widetilde{m}}{1-y_{t}} f_{t}(1-\tau) .
$$

Since $\frac{\phi_{1}}{\phi_{2}} \approx 0$, the aggregate endowment follows a geometric Brownian motion in the limit, and the interest rate is constant $r_{t}=r$. Accordingly, the price of a stock of type 1 follows the dynamics

$$
\frac{d P_{1, t, s}+D_{1, t, s} d t}{P_{1, t, s}}=\left(r+\kappa_{1, t} \sigma_{1, t}\right) d t+\sigma_{t} d B_{1, t} .
$$

Applying Ito's Lemma to the product $P_{1, t, s}=p\left(\omega_{t}\right) D_{1, t, s}$ also implies that

$$
\frac{d P_{1, t, s}}{P_{1, t, s}}=\frac{d p_{t}}{p_{t}}+\frac{d D_{1, t, s}}{D_{1, t, s}}+\frac{p^{\prime}\left(\omega_{t}\right)}{p\left(\omega_{t}\right)} \sigma_{t}^{R} \sigma_{1, D} d t
$$

\footnotetext{
${ }^{32}$ The reason is that the price-dividend ratio and the ratio of the dividend processes for the two trees (given in (43)) approach constants, thus implying that $\widetilde{m}_{t}=\widetilde{m}$ approaches a constant.

${ }^{33} \mathrm{Using} \sum_{i \in\{I, R\}} w_{1, t}^{i} \omega_{t}^{i}=\widetilde{m}_{t}$, the definition $y_{t}=-\frac{w_{1, t}^{R} \omega_{t} 1_{\left\{w_{1, t}^{R}<0\right\}}}{w_{1, t}^{I} \omega_{t}^{I}}$ and the definition of $\lambda_{t}^{i}$ leads to$$
-\sum_{i \in\{I, R\}} w_{1, t}^{i} \omega_{t}^{i} \lambda_{t}^{i}=\frac{y_{t} \widetilde{m}}{1-y_{t}} f_{t}(1-\tau) .
$$ 
Combining C.3 with C.4 and using $\sigma_{1, t}=\frac{p^{\prime}\left(\omega_{t}\right)}{p\left(\omega_{t}\right)} \sigma_{t}^{R}+\sigma_{1, D}$ and Ito's Lemma to compute the drift of $\frac{d p_{t}}{p_{t}}$ leads to

$$
\frac{1}{2} \frac{\partial^{2} p}{\partial \omega_{t}^{2}}\left(\sigma_{t}^{R}\right)^{2}+\frac{\partial p}{\partial \omega_{t}}\left(\mu_{t}^{R}+\sigma_{t}^{R} \sigma_{1, D}\right)-p \times\left(r+\delta_{1}+\kappa_{1, t} \sigma_{1, t}\right)+1=0
$$

which in turn leads to (48) after substituting $\sigma_{1, t}=\frac{p^{\prime}\left(\omega_{t}\right)}{p\left(\omega_{t}\right)} \sigma_{t}^{R}+\sigma_{1, D}$.

We solve (48) with iterated Monte Carlo. We start with the initial guess $\sigma_{1, t}=\sigma_{1, D}$ and some guess for the cutoff $\bar{\omega}$. With that guess we use a Monte carlo simulation to evaluate $V_{t}^{R}\left(\omega_{t}\right)$ on a grid of $\omega_{t}$ values. We find the value that implies $V_{t}^{R}\left(\omega_{t}\right)=0$ and update our guess for $\bar{\omega}$ until $V_{t}^{R}(\bar{\omega})=0$. With this guess for $\bar{\omega}$ we draw paths of $\omega_{t}$ for different initial values and solve for the price-dividend ratio by using the Feynman-Kac theorem to express (48) as an expectation, which we evaluate with Monte Carlo simulation. After obtaining the pricedividend ratio on a fine grid of values, we evaluate $\frac{p^{\prime}\left(\omega_{t}\right)}{p\left(\omega_{t}\right)}$, and compute $\sigma_{1, t}=\frac{p^{\prime}\left(\omega_{t}\right)}{p\left(\omega_{t}\right)} \sigma_{t}^{R}+\sigma_{1, D}$. Using this new guess for $\sigma_{1, t}$ we repeat the above procedure until convergence.

\section{Generalized supply curve for shorting}

The baseline version of the model assumes an elastic supply of lendable shares, so that the lending fee is constant. The results generalize readily to the case in which the supply of lendable shares is increasing in $y_{t}$ so that $f_{t}=l\left(y_{t}\right)$, where $l^{\prime}\left(y_{t}\right)>0$.

We obtain the following proposition.

Proposition 7 Consider the model of Section 2, but without Assumption 1. Define

$$
z\left(y_{t}\right) \equiv f_{t}\left(1-\tau y_{t}\right)=l\left(y_{t}\right)\left(1-\tau y_{t}\right)
$$

Assume that $\frac{\sigma_{D}}{1-\omega_{t}}-\eta<0$ and that there exists $y_{t}^{*} \in(0,1)$ such that

$$
y_{t}^{*}=\frac{\eta-\frac{\sigma_{D}}{1-\omega_{t}}-\frac{1}{\sigma_{D}} z\left(y_{t}^{*}\right)}{\eta+\frac{\sigma_{D}}{\omega_{t}}-\frac{1}{\sigma_{D}} z\left(y_{t}^{*}\right)}
$$

and $\eta-\frac{\sigma_{D}}{1-\omega_{t}}-\frac{1}{\sigma_{D}} z\left(y^{*}\right)>0$. Moreover, if $\eta-\frac{\sigma_{D}}{1-\omega_{t}}-\frac{1}{\sigma_{D}} z(0)<0$, then there exist at least two values of $y_{t}^{(j)}, j=\{1,2\}$ satisfying both (D.2) and $\eta-\frac{\sigma_{D}}{1-\omega_{t}}-\frac{1}{\sigma_{D}} z\left(y^{(j)}\right)>0$ and three equilibria co-exist. In the first equilibrium, the $R$ investor holds a zero portfolio, and the Sharpe ratio is $\kappa_{t}=\frac{\sigma_{D}}{1-\omega_{t}}-\eta$. There also exist two other equilibria, with $R$ investors holding negative portfolios and the Sharpe ratio given by

$$
\kappa_{t}=\sigma_{D}-\left(1-\omega_{t}\right) \eta-\frac{\omega_{t}}{\sigma_{D}} l\left(y_{t}^{(j)}\right)\left[1+\tau y_{t}^{(j)} \frac{1-\omega_{t}}{\omega_{t}}\right]
$$

In all equilibria the interest rate is given by (26), and the lending fee is given by $l\left(y_{t}^{(j)}\right)$.

Remark 3 In the special case $l\left(y_{t}\right)=\varphi$, equations (D.2) and (D.3) become identical to (24) and (25), respectively. 


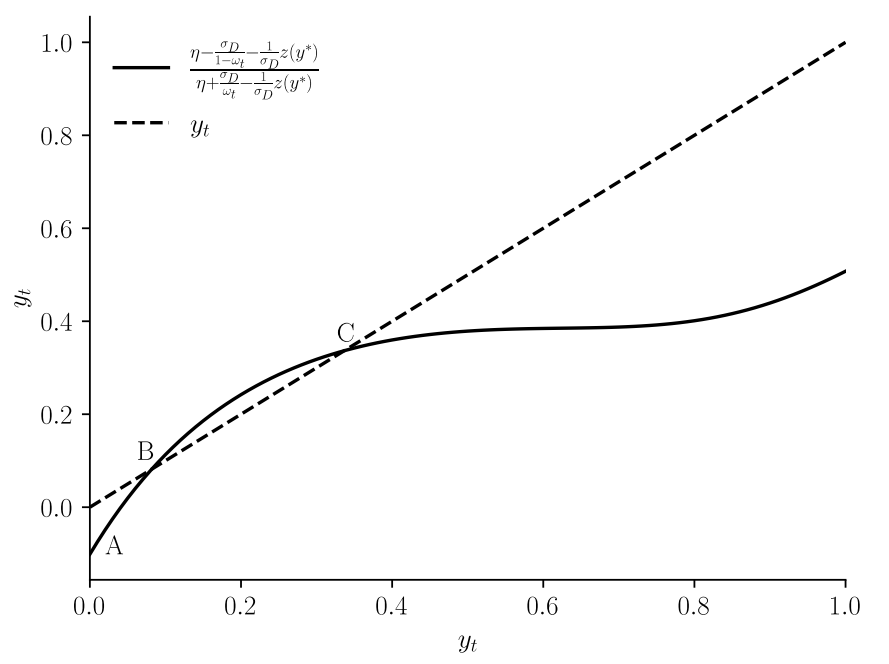

Figure 13: An illustration of Proposition 7.

Figure 13 illustrates Proposition 7. For this particular numerical example we choose $l\left(y_{t}\right)=\varphi\left(1+2 y_{t}^{3}\right), \eta=0.9, \sigma_{D}=7 \%, \tau=0.8, \omega=0.5$, and $\varphi=0.055$. The figure plots the left hand side (dotted line) of equation (D.2) and the right hand side (solid line). Points $B$ and $C$ correspond to the two fixed points. Point $A$ in the figure illustrates the assumption $\eta-\frac{\sigma_{D}}{1-\omega_{t}}-\frac{1}{\sigma_{D}} z(0)<0$. This inequality implies that there is a third equilibrium in which $R$ investors choose to not go short. The fees in the three equilibria differ, with the lending fee being lowest $(l(0)=\varphi)$ in equilibrium $A$, in which the shorting market is inactive, and highest in equilibrium $C$, in which $y$ is highest.

\section{E Proofs}

Proof of Proposition 1. Fix parameters $\eta>0$ and $\psi>1$ and define $\varphi$ according to

$$
\varphi=\sigma_{D}\left(\eta-\psi \sigma_{D}\right)
$$

for any value of $\sigma_{D}$. Note that when $\sigma_{D}$ is sufficiently small, $\varphi$ is guaranteed to be positive.

We show next that, as $\sigma_{D}$ gets close to zero, Assumption 2 is satisfied. Rearranging (E.1) gives

$$
\frac{\eta}{\frac{\varphi}{\sigma_{D}}}=\frac{1}{1-\psi \frac{\sigma_{D}}{\eta}} .
$$

For sufficiently small $\sigma_{D}$ we obtain

$$
1+\tau>\frac{1}{1-\psi \frac{\sigma_{D}}{\eta}}>1 .
$$


Combining (E.2) and (E.3) yields (21).

Turning to (22), we note that the definition of $\omega_{1}^{*}$ along with (E.1) implies

$$
\omega_{1}^{*}=1-\frac{\sigma_{D}}{\psi \sigma_{D}}=\frac{\psi-1}{\psi}>0,
$$

while also

$$
\lim _{\sigma_{D} \rightarrow 0} \frac{\sigma_{D}}{(1+\tau) \frac{\varphi}{\sigma_{D}}-\eta}=\lim _{\sigma_{D} \rightarrow 0} \frac{\sigma_{D}}{(1+\tau)\left(\eta-\psi \sigma_{D}\right)-\eta}=0 .
$$

Therefore, for sufficiently small $\sigma_{D}$, the left-hand side of 22 converges to $\frac{\psi-1}{\psi}>0$, while the right-hand side converges to zero, and therefore the inequality holds.

We conclude the proof by showing that $F(\omega)$ has a unique root in the interval $\left(\omega_{1}^{*}, 1\right)$. To this end, it is useful to introduce the definitions

$$
\begin{aligned}
A(\omega) & \equiv \tau \frac{\omega}{\sigma_{D}} \varphi, \\
B(\omega) & \equiv \sigma_{D}-\omega\left((1+\tau) \frac{\varphi}{\sigma_{D}}-\eta\right), \\
C(\omega) & \equiv \frac{\omega}{1-\omega}\left(\sigma_{D}+(1-\omega)\left(\frac{\varphi}{\sigma_{D}}-\eta\right)\right) .
\end{aligned}
$$

With these definitions, $F(\omega)$ can be written as $F(\omega)=B^{2}(\omega)-4 A(\omega) C(\omega)$. We start by observing that $C\left(\omega_{1}^{*}\right)=0$ for any parametric choice (since the definition of $\omega_{1}^{*}$ in equation 19 implies $\left.\sigma_{D}+\left(1-\omega_{1}^{*}\right)\left(\frac{\varphi}{\sigma_{D}}-\eta\right)=0\right)$. Also, inequality 22 implies that $B\left(\omega_{1}^{*}\right) \neq 0$, and thus $B^{2}\left(\omega_{1}^{*}\right)>0$. Accordingly, $F\left(\omega_{1}^{*}\right)>0$. Also $B(1)<\infty$, while $C(1)=\infty$. By continuity, there exists at least one value $\omega_{2}^{*} \in\left(\omega_{1}^{*}, 1\right)$ such that $F\left(\omega_{2}^{*}\right)=0$.

To show that this value is unique, consider any value $\omega_{2}^{*} \in\left(\omega_{1}^{*}, 1\right)$ such that $F\left(\omega_{2}^{*}\right)=0$. We next show that $F^{\prime}\left(\omega_{2}^{*}\right)<0$.

To this end, note that

$$
\begin{aligned}
F^{\prime}(\omega) & =2 B(\omega) B^{\prime}(\omega)-4\left[A^{\prime}(\omega) C(\omega)+A(\omega) C^{\prime}(\omega)\right] \\
& =2 B^{2}(\omega) \frac{B^{\prime}(\omega)}{B(\omega)}-4 A(\omega) C(\omega)\left(\frac{A^{\prime}(\omega)}{A(\omega)}+\frac{C^{\prime}(\omega)}{C(\omega)}\right) .
\end{aligned}
$$

Since $\omega_{2}^{*}$ is a root of $F(\omega)$ it follows that $B^{2}\left(\omega_{2}^{*}\right)=4 A\left(\omega^{(2)}\right) C\left(\omega_{2}^{*}\right)$. Therefore,

$$
F^{\prime}\left(\omega_{2}^{*}\right)=B^{2}\left(\omega_{2}^{*}\right)\left(2 \frac{B^{\prime}\left(\omega_{2}^{*}\right)}{B\left(\omega_{2}^{*}\right)}-\frac{A^{\prime}\left(\omega_{2}^{*}\right)}{A\left(\omega_{2}^{*}\right)}-\frac{C^{\prime}\left(\omega_{2}^{*}\right)}{C\left(\omega_{2}^{*}\right)}\right) .
$$


We have

$$
\begin{aligned}
& \frac{A^{\prime}\left(\omega_{2}^{*}\right)}{A\left(\omega_{2}^{*}\right)}=\frac{1}{\omega_{2}^{*}} \\
& \frac{B^{\prime}\left(\omega_{2}^{*}\right)}{B\left(\omega_{2}^{*}\right)}=-\frac{(1+\tau) \frac{\varphi}{\sigma_{D}}-\eta}{\sigma_{D}-\omega_{2}^{*}\left((1+\tau) \frac{\varphi}{\sigma_{D}}-\eta\right)}
\end{aligned}
$$

and

$$
\frac{C^{\prime}\left(\omega_{2}^{*}\right)}{C\left(\omega_{2}^{*}\right)}=\frac{1}{\omega_{2}^{*}\left(1-\omega_{2}^{*}\right)}+\frac{\eta-\frac{\varphi}{\sigma_{D}}}{\sigma_{D}+\left(1-\omega_{2}^{*}\right)\left(\frac{\varphi}{\sigma_{D}}-\eta\right)}
$$

Combining terms gives

$$
\begin{aligned}
& 2 \frac{B^{\prime}\left(\omega_{2}^{*}\right)}{B\left(\omega_{2}^{*}\right)}-\frac{A^{\prime}\left(\omega_{2}^{*}\right)}{A\left(\omega_{2}^{*}\right)}-\frac{C^{\prime}\left(\omega_{2}^{*}\right)}{C\left(\omega_{2}^{*}\right)} \\
& \quad=-\frac{2\left((1+\tau) \frac{\varphi}{\sigma_{D}}-\eta\right)}{\sigma_{D}-\omega_{2}^{*}\left((1+\tau) \frac{\varphi}{\sigma_{D}}-\eta\right)}-\frac{1}{\omega_{2}^{*}}-\frac{1}{\omega_{2}^{*}\left(1-\omega_{2}^{*}\right)}-\frac{\eta-\frac{\varphi}{\sigma_{D}}}{\sigma_{D}+\left(1-\omega_{2}^{*}\right)\left(\frac{\varphi}{\sigma_{D}}-\eta\right)} .
\end{aligned}
$$

For future reference, we note that using $\omega_{2}^{*}>\omega_{1}^{*}$ along with (21) and the definition of $\omega_{1}^{*}$ implies that

$$
\sigma_{D}+\left(1-\omega_{2}^{*}\right)\left(\frac{\varphi}{\sigma_{D}}-\eta\right)>\sigma_{D}+\left(1-\omega_{1}^{*}\right)\left(\frac{\varphi}{\sigma_{D}}-\eta\right)=0
$$

Using (E.1) we can write the right-hand side of (E.8) as

$$
-\frac{2\left((1+\tau)\left(\eta-\psi \sigma_{D}\right)-\eta\right)}{\sigma_{D}-\omega_{2}^{*}\left((1+\tau)\left(\eta-\psi \sigma_{D}\right)-\eta\right)}-\frac{1}{\omega_{2}^{*}}-\frac{1}{\omega_{2}^{*}\left(1-\omega_{2}^{*}\right)}-\frac{\psi}{1-\psi\left(1-\omega_{2}^{*}\right)} .
$$

Taking the limit as $\sigma_{D}$ approaches zero, the expression (E.10) converges to

$$
-\frac{1}{1-\omega_{2}^{*}}-\frac{\psi}{1-\psi\left(1-\omega_{2}^{*}\right)}<0
$$

where the inequality follows from $\left(\right.$ E.9 along with $\left(\right.$ E.1 ${ }^{34}$

The fact that the derivative $F^{\prime}\left(\omega_{2}^{*}\right)<0$ for any root of the equation $F\left(\omega_{2}^{*}\right)=0$ in the interval $\left(\omega_{1}^{*}, 1\right)$ implies that the root $\omega_{2}^{*}$ must be unique.

Proof of Proposition 2. In preparation for the proof, we state and prove an auxiliary result.

\footnotetext{
${ }^{34}$ Equation E.1) implies $\frac{\varphi}{\sigma_{D}}-\eta=-\psi \sigma_{D}$, and therefore $0<\sigma_{D}+\left(1-\omega_{2}^{*}\right)\left(\frac{\varphi}{\sigma_{D}}-\eta\right)=\sigma_{D}\left(1-\left(1-\omega_{2}^{*}\right) \psi\right)$, where the inequality follows from $($ E.9. .
} 
Lemma 2 The following statements hold for the quadratic equation (24).

1. $\omega_{1}^{*}<\omega_{2}^{*}$ and the discriminant of (24) is non-negative for all $\omega_{t} \leq \omega_{2}^{*}$.

2. When $\omega_{1}^{*} \leq \omega_{t} \leq \omega_{2}^{*}$, the two roots of the equation are both in the interval $[0,1)$.

3. For $\omega_{t} \in\left[0, \omega_{1}^{*}\right)$, only the larger root of (24) is in the interval $(0,1)$.

4. If $y$ is a root of (24), then $\left(1-\omega_{t}\right) \eta-\sigma_{D}-\frac{1-\omega_{t}}{\sigma_{D}} \varphi(1-\tau y)>0$.

Proof of Lemma 2. We start with part 1. Using the definitions (E.4)-(E.6), equation (24) can be written in the familiar form

$$
A\left(\omega_{t}\right) y^{2}+B\left(\omega_{t}\right) y+C\left(\omega_{t}\right)=0,
$$

and the discriminant of this quadratic equation is given by $F\left(\omega_{t}\right)$ as defined in equation (20).

For $\omega_{t} \leq \omega_{1}^{*}, C\left(\omega_{t}\right)<0$ and the discriminant, $B^{2}\left(\omega_{t}\right)-4 A\left(\omega_{t}\right) C\left(\omega_{t}\right)$, is positive. The assumption that $\omega_{2}^{*}$ is the unique root of $F(\omega)$ along with the facts that $F\left(\omega_{1}^{*}\right)=B^{2}\left(\omega_{1}^{*}\right)>0$ and $F(1)=-\infty$ imply that $\omega_{1}^{*}<\omega_{2}^{*}{ }^{35}$ The uniqueness of the root $\omega_{2}^{*}$ also implies that $F\left(\omega_{t}\right)=B^{2}\left(\omega_{t}\right)-4 A\left(\omega_{t}\right) C\left(\omega_{t}\right) \geq 0$ for all $\omega_{t} \leq \omega_{2}^{*}$.

We now turn to part 2 . To economize on notation we write $A$ rather $A\left(\omega_{t}\right)$ and similarly for $B$ and $C$. Fix a given $\omega_{t}$ and let $g(y)=A y^{2}+B y+C$. We have $g(1)=A+B+C=$ $\frac{\sigma_{D}}{1-\omega_{t}}>0$ and $g^{\prime}(1)=2 A+B=\sigma_{D}+\omega_{t}\left(\eta-(1-\tau) \frac{\varphi}{\sigma_{D}}\right)>0$, where the inequality follows from (21). Since $A>0$, it follows that all roots of $g(y)$ must be smaller than one. Also, the fact that $\omega_{t} \geq \omega_{1}^{*}$ implies that $g(0)=C>0$, while assumptions (21) and (22) together with the fact that $\omega_{t} \geq \omega_{1}^{*}$ imply that $g^{\prime}(0)=B<0$.

The facts that i) $g(y)$ is a convex, quadratic function of $y$, ii) $g(1)>0, g(0)>0$, $g^{\prime}(1)>0$, and $g^{\prime}(0)<0$ and iii) $B^{2}-4 A C>0$ for $\omega_{t} \in\left[\omega_{1}^{*}, \omega_{2}^{*}\right)$ imply that there are two roots in $(0,1)$.

For part 3. we note that, when $\omega_{t}<\omega_{1}^{*}, g(0)=C<0$, while $g(1)=A+B+C=\frac{\sigma_{D}}{1-\omega_{t}}>0$. Therefore there exists one and only one root in $(0,1)$.

Finally, let $y \in(0,1)$ denote a root of the quadratic equation (24). Accordingly,

$$
\begin{aligned}
\left(1-\omega_{t}\right) \eta-\sigma_{D}-\left(1-\omega_{t}\right) \frac{\varphi}{\sigma_{D}}(1-\tau y) & =\frac{1-\omega_{t}}{\omega_{t}} y\left(\sigma_{D}+\omega_{t} \eta-\omega_{t} \frac{\varphi}{\sigma_{D}}(1-\tau y)\right) \\
& =\frac{1-\omega_{t}}{\omega_{t}} y\left[\sigma_{D}+\omega_{t}\left(\eta-\frac{\varphi}{\sigma_{D}}\right)+\omega_{t} \frac{\varphi}{\sigma_{D}} \tau y\right] \\
& >0
\end{aligned}
$$

where the last inequality follows from (21). This proves property 4 .

We now continue with the proof of the proposition. We provide expressions for $r_{t}$ and $\kappa_{t}$ that apply in any equilibrium in which $w_{t}^{R} \neq 0$. Since $\sum_{i} \omega_{t}^{i}=1$, it follows that $\sum_{i} \sigma_{t}^{i}=0$

${ }^{35}$ Assumption 22 implies that $B\left(\omega_{1}^{*}\right) \neq 0$ and therefore $B^{2}\left(\omega_{1}^{*}\right)>0$. 
and $\sum_{i} \mu_{t}^{i}=0$. Using (17) and $\sum_{i} \sigma_{t}^{i}=0$ implies that $\sum_{i} \omega_{t}^{i} w_{t}^{i}=1$. Combining $\sum_{i} \omega_{t}^{i} w_{t}^{i}=1$ with 14 along with the definition $y_{t}=\frac{W_{t}^{-}}{W_{t}^{+}}$gives

$$
\kappa_{t}+\left(1-\omega_{t}\right) \eta+\left(\omega_{t} \frac{1}{\sigma_{D}} \varphi+\left(1-\omega_{t}\right) \tau y_{t} \frac{1}{\sigma_{D}} \varphi\right) 1_{\left\{w_{t}^{R}<0\right\}}=\sigma^{D} .
$$

Similarly, using (18) along with $\sum_{i} \mu_{t}^{i}=0$ and $\sum_{i} \omega_{t}^{i}\left(n_{t}+w_{t}^{i} s_{t}^{i}\right)=0$ gives (26).

We next describe the equilibria for the three intervals of $\omega_{t}$ described in the statement of the proposition.

i) In this case, $\omega_{t}>\omega_{2}^{*}$. The equilibrium prescribes non-negative portfolios for both investors. If $\omega_{t}>1-\frac{\sigma_{D}}{\eta}$, equation (E.11) implies that $\kappa_{t}>0$ and (14) implies that both investors hold positive portfolios and the shorting market is inactive. If $\omega_{t} \in\left[\omega_{1}^{*}, 1-\frac{\sigma_{D}}{\eta}\right)$, then there exists an equilibrium that involves no shorting and a zero portfolio for investor $R$. We check this assertion by osberving that the associated market clearing requirement becomes $\left(1-\omega_{t}\right) w_{t}^{I}=1$, which together with $y_{t}=0$ leads to $(23)$. We then note that

$$
\begin{aligned}
\kappa_{t}+\frac{\varphi}{\sigma_{D}} & =\frac{\sigma_{D}}{1-\omega_{t}}-\eta+\frac{\varphi}{\sigma_{D}} \\
& >\frac{\sigma_{D}}{1-\omega_{1}^{*}}-\eta+\frac{\varphi}{\sigma_{D}} \\
& =0 .
\end{aligned}
$$

The first line follows from $(23)$, the second line follows from $\omega_{t}>\omega_{1}^{*}$ and the third line follows from the definition of $\omega_{1}^{*}$. Since $\kappa_{t}+\frac{\varphi}{\sigma_{D}}>0$, investor $R$ does not choose a negative portfolio. And since $\kappa_{t}<0$ for $\omega_{t} \in\left[\omega_{1}^{*}, 1-\frac{\sigma_{D}}{\eta}\right)$, the investor chooses a zero portfolio.

ii) In this case, $\omega_{1}^{*}<\omega_{t}<\omega_{2}^{*}$. Since $\omega_{t}>\omega_{1}^{*}$, equation E.12 implies that the no-shorting equilibrium continues to be an equilibrium. There exist, however, two more equilibria. To compute them, we guess (and verify shortly) that $w_{t}^{R}<0$. Using (14) and E.11) gives

$$
\begin{aligned}
y_{t} & =\frac{W_{t}^{-}}{W_{t}^{+}}=\frac{-\omega_{t} w_{t, s}^{R}}{\left(1-\omega_{t}\right) w_{t, s}^{I}}=\frac{\omega_{t}}{1-\omega_{t}} \frac{-\left(\kappa_{t}+\frac{1}{\sigma_{D}} \varphi\right)}{\kappa_{t}+\eta_{t}+\frac{1}{\sigma_{D}} \varphi \tau y_{t}} \\
& =\frac{\omega_{t}}{1-\omega_{t}} \frac{\left(1-\omega_{t}\right) \eta-\sigma_{D}-\frac{1-\omega_{t}}{\sigma_{D}} \varphi\left(1-\tau y_{t}\right)}{\sigma_{D}+\omega_{t} \eta-\frac{\omega_{t}}{\sigma_{D}} \varphi\left(1-\tau y_{t}\right)} .
\end{aligned}
$$

Rearranging leads to (24). Statement 1 of Lemma 2 implies that, when $\omega_{t} \in\left(\omega_{1}^{*}, \omega_{2}^{*}\right)$, equation 24 has two roots in $(0,1)$. Under the supposition that $w_{t}^{R}<0$, Equation 
(E.11) leads to 25). In turn

$$
\begin{aligned}
\kappa_{t}^{ \pm}+\frac{\varphi}{\sigma_{D}} & =\sigma_{D}-\left(1-\omega_{t}\right) \eta-\frac{\omega_{t}}{\sigma_{D}} \varphi\left(1+\tau y^{ \pm} \frac{1-\omega_{t}}{\omega_{t}}\right)+\frac{\varphi}{\sigma_{D}} \\
& =\sigma_{D}-\left(1-\omega_{t}\right)\left(\eta+\frac{\varphi}{\sigma_{D}}\left(1-\tau y_{t}^{ \pm}\right)\right)<0,
\end{aligned}
$$

where the last inequality follows from statement 4 of Lemma 2. Combining this observation with (14) confirms that $w_{t}^{R}<0$. Note that in the second and third equilibria we have that

$$
\kappa_{t}^{ \pm}+\eta_{t}+\frac{1}{\sigma_{D}} \varphi \tau y_{t}^{ \pm}=\sigma_{D}+\omega_{t} \eta-\frac{\varphi \omega_{t}}{\sigma_{D}}\left(1-\tau y_{t}^{ \pm}\right)>0
$$

where the last inequality follows from (E.13) along with the fact that $y^{ \pm}$satisfy the equation (24). This implies that $w_{t}^{I}>0$.

iii) In this case, $\omega_{t}<\omega_{1}^{*}$. Statement 3 of Lemma 2 implies that the quadratic equation (24) has only one solution in $(0,1)$. This shows that there can only be one equilibrium with shorting. Moreover, this is the unique equilibrium. If $w_{t}^{R}$ were zero and the Sharpe ratio were $\frac{\sigma_{D}}{1-\omega_{t}}-\eta$, then the inequality in $\left(\mathrm{E} .12\right.$ reverses, i.e., $\frac{\sigma_{D}}{1-\omega_{t}}-\eta+\frac{\varphi}{\sigma_{D}}<0$ and investor $R$ would want to deviate from the equilibrium prescription and choose a negative portfolio.

Proof of Proposition 3. We distinguish two cases according to whether investor $R$ holds an interior positions in both equilbria.

Case i: Suppose that $w_{t}^{R, A}=0$ in equilibrium $A$ and $w_{t}^{R, B}<0$ in equilibrium $B$. We have

$$
\begin{aligned}
g_{t}^{R, B}-g_{t}^{R, A} & =-\left(\kappa^{B}-\kappa^{A}\right) \sigma_{D}+\max _{w_{t} \leq 0}\left\{w_{t}\left(\kappa^{B} \sigma_{D}+\varphi\right)-\frac{1}{2} w_{t}^{2} \sigma_{t}^{2}\right\} \\
& >\left(\kappa^{A}-\kappa^{B}\right) \sigma_{D} \geq 0
\end{aligned}
$$

where the first inequality follows from the fact that $w_{t}=0$ is suboptimal for investor $R$ in equilibrium $B$ (by assumption). Similarly, using (18) gives

$$
\begin{aligned}
\mu_{t}^{R, B}-\mu_{t}^{R, A} & =\omega_{t}\left(\left(\kappa^{A}-\kappa^{B}\right) \sigma_{D}+w_{t}^{R, B} \sigma_{D}\left(\kappa^{B}+\frac{\varphi}{\sigma_{D}}-\sigma_{D}\right)\right) \\
& =\omega_{t}\left[\left(\kappa^{A}-\kappa^{B}\right) \sigma_{D}+\left(1-\omega_{t}\right) w_{t}^{R, B} \sigma_{D}\left(\frac{\varphi}{\sigma_{D}}(1-y)-\eta\right)\right] \\
& =\omega_{t}\left[\left(\kappa^{A}-\kappa^{B}\right) \sigma_{D}+\left(1-\omega_{t}\right)\left|w_{t}^{R, B}\right| \sigma_{D}\left(\eta-\frac{\varphi}{\sigma_{D}}(1-y)\right)\right] \\
& >0
\end{aligned}
$$

where the first equality follows from 25 , the second equality from $w_{t}^{R, B}<0$ and the inequality from assumption (21) along with $y<1$. 
Case ii: In this case the portfolio choice of investor $R$ is interior in both equilibria. Using the fact that in any interior equilibrium the optimal value of $w_{t}$ satisfies

$$
w_{t}\left(\kappa^{B} \sigma_{D}+\varphi\right)-\frac{1}{2} w_{t}^{2} \sigma_{D}^{2}=\frac{1}{2} w_{t}^{2} \sigma_{D}^{2},
$$

we obtain

$$
\begin{aligned}
g_{t}^{R, B}-g_{t}^{R, A} & =-\left(\kappa^{B}-\kappa^{A}\right) \sigma_{D}+\frac{\sigma_{D}^{2}}{2}\left[\left(w_{t}^{R, B}\right)^{2}-\left(w_{t}^{R, A}\right)^{2}\right] \\
& =\left(\kappa^{A}-\kappa^{B}\right) \sigma_{D}+\frac{\sigma_{D}^{2}}{2}\left(w_{t}^{R, B}+w_{t}^{R, A}\right)\left(w_{t}^{R, B}-w_{t}^{R, A}\right) \\
& =\left(\kappa^{A}-\kappa^{B}\right) \sigma_{D}+\frac{\sigma_{D}}{2}\left(w_{t}^{R, B}+w_{t}^{R, A}\right)\left(\kappa^{B}-\kappa^{A}\right) \\
& =\left(\kappa^{A}-\kappa^{B}\right) \sigma_{D}\left(1+\left|w_{t}^{R, B}+w_{t}^{R, A}\right|\right) \\
& >0 .
\end{aligned}
$$

Using (18) gives

$$
\begin{aligned}
\mu_{t}^{R, B}-\mu_{t}^{R, A} & =\omega_{t}\left(\left(\kappa^{A}-\kappa^{B}\right) \sigma_{D}+w_{t}^{R, B} \sigma_{D}\left(\kappa^{B}+\frac{\varphi}{\sigma_{D}}-\sigma_{D}\right)-w_{t}^{R, A} \sigma_{D}\left(\kappa^{A}+\frac{\varphi}{\sigma_{D}}-\sigma_{D}\right)\right) \\
& =\omega_{t}\left(\left(\kappa^{A}-\kappa^{B}\right) \sigma_{D}+\sigma_{D}^{2}\left[w_{t}^{R, B}\left(w_{t}^{R, B}-1\right)-w_{t}^{R, A}\left(w_{t}^{R, A}-1\right)\right]\right) \\
& =\omega_{t}\left(\left(\kappa^{A}-\kappa^{B}\right) \sigma_{D}+\sigma_{D}^{2}\left[\left(w_{t}^{R, B}-\frac{1}{2}\right)^{2}-\left(w_{t}^{R, A}-\frac{1}{2}\right)^{2}\right]\right) \\
& =\omega_{t}\left(\left(\kappa^{A}-\kappa^{B}\right) \sigma_{D}+\sigma_{D}^{2}\left[\left(\left|w_{t}^{R, B}\right|+\frac{1}{2}\right)^{2}-\left(\left|w_{t}^{R, A}\right|+\frac{1}{2}\right)^{2}\right]\right) \\
& >0
\end{aligned}
$$

where the last inequality follows from $w_{t}^{R, B}<w_{t}^{R, A}<0$ (since $\kappa^{B}<\kappa^{A}<0$ ) and therefore $\left|w_{t}^{R, B}\right|>\left|w_{t}^{R, A}\right|$.

Proof of Lemma 1. By the implicit function theorem,

$$
\frac{d \omega_{2}^{*}}{d \varphi}=-\frac{F_{\varphi}}{F_{\omega}} .
$$

Since $\lim _{\omega \rightarrow \infty} F(\omega)=-\infty$ and the root $F\left(\omega_{2}^{*}\right)=0$ is unique (by assumption), it follows that $F_{\omega}\left(\omega_{2}^{*}\right)<0$. So it suffices to prove that $F_{\varphi}\left(\omega_{2}^{*}\right)<0$.

Differentiating $F$ with respect to $\varphi$, multiplying the resulting expression by $\varphi$ and eval- 
uating at $\omega_{2}^{*}\left(\right.$ recall $\left.F\left(\omega_{2}^{*}\right)=0\right)$ gives

$$
\begin{aligned}
\varphi F_{\varphi}= & -2 \omega_{2}^{*} \frac{\varphi}{\sigma_{D}}(1+\tau)\left(\sigma_{D}-\omega_{2}^{*}\left((1+\tau) \frac{\varphi}{\sigma_{D}}-\eta\right)\right) \\
& -\left(\sigma_{D}-\omega_{2}^{*}\left((1+\tau) \frac{\varphi}{\sigma_{D}}-\eta\right)\right)^{2}-4 \tau\left(\omega_{2}^{*}\right)^{2} \frac{\varphi^{2}}{\sigma_{D}^{2}}
\end{aligned}
$$

Completing the square gives

$$
\begin{aligned}
\varphi F_{\varphi} & =-\left(\sigma_{D}-\omega_{2}^{*}\left((1+\tau) \frac{\varphi}{\sigma_{D}}-\eta\right)+\omega_{2}^{*} \frac{\varphi}{\sigma_{D}}(1+\tau)\right)^{2}+\left(\omega_{2}^{*}\right)^{2} \frac{\varphi^{2}}{\sigma_{D}^{2}}(1-\tau)^{2} \\
& =-\left(\sigma_{D}+\omega_{2}^{*} \eta\right)^{2}+\left(\omega_{2}^{*}\right)^{2} \frac{\varphi^{2}}{\sigma_{D}^{2}}(1-\tau)^{2} \\
& =-\left(\sigma_{D}+\omega_{2}^{*} \eta+\omega_{2}^{*} \frac{\varphi}{\sigma_{D}}(1-\tau)\right)\left(\sigma_{D}+\omega_{2}^{*} \eta-\omega_{2}^{*} \frac{\varphi}{\sigma_{D}}(1-\tau)\right) \\
& <0
\end{aligned}
$$

where the last inequality follows from the assumption $\eta \geq \frac{\varphi}{\sigma_{D}}$.

Proof of Proposition 4. Differentiating $\kappa_{t}$ with respect to $\varphi$ (in an equilibrium where $y>0$ ), we obtain

$$
\frac{d \kappa_{t}}{d \varphi}=-\frac{\omega_{t}}{\sigma_{D}}\left(1+\frac{1-\omega_{t}}{\omega_{t}} \tau y_{t}\left(1+\frac{\varphi}{y_{t}} \frac{d y_{t}}{d \varphi}\right)\right)
$$

In turn, the implicit function theorem applied to (24) gives

$$
\frac{d y_{t}}{d \varphi}=-\frac{\frac{\omega_{t}}{\sigma_{D}}(1-\tau y)(1-y)}{\sigma_{D}+\omega_{t} \eta-\frac{\omega_{t}}{\sigma_{D}} \varphi(1+\tau-2 \tau y)}=-\frac{\frac{\omega_{t}}{\sigma_{D}}(1-\tau y)(1-y)}{g^{\prime}(y)},
$$

where $g(y)$ is defined in Proposition 1. Since $g^{\prime}\left(y^{-}\right)<0$ and $g^{\prime}\left(y^{+}\right)>0$, we have $\frac{d y^{-}}{d \varphi}>0$ and $\frac{d y^{+}}{d \varphi}<0$. Combining $\frac{d y^{-}}{d \varphi}>0$ with E.15 implies $\frac{d \kappa_{t}}{d \varphi}<0$ in the equilibrium associated with $y^{-}$. For the equilibrium associated with $y^{+}$we have

$$
1+\frac{\varphi}{y^{+}} \frac{d y^{+}}{d \varphi}=\frac{\left(\sigma_{D}+\omega_{t} \eta-\frac{\omega_{t}}{\sigma_{D}} \varphi\left(1+\tau-2 \tau y^{+}\right)\right) y^{+}-\frac{\varphi \omega_{t}}{\sigma_{D}}\left(1-\tau y^{+}\right)\left(1-y^{+}\right)}{\left(\sigma_{D}+\omega_{t} \eta-\frac{\omega_{t}}{\sigma_{D}} \varphi\left(1+\tau-2 \tau y^{+}\right)\right) y^{+}}
$$

We are interested in the behavior of $\left(\right.$ E.16) as $\omega_{t}$ approaches zero. Letting $x \equiv \frac{y^{+}}{\omega_{t}}$, dividing both sides of (24) by $\omega_{t}$ and re-arranging terms yields

$$
x\left(\sigma_{D}+\omega_{t} \eta\right)+\frac{\varphi}{\sigma_{D}}\left(1-\omega_{t} x\right)\left(1-\tau \omega_{t} x\right)=\frac{1}{1-\omega_{t}}\left(\left(1-\omega_{t}\right) \eta^{I}-\sigma_{D}\right) .
$$


Taking limits as $\omega_{t}$ approaches zero, implies

$$
\lim _{\omega_{t} \rightarrow 0} x=\frac{\eta-\sigma_{D}-\frac{\varphi}{\sigma_{D}}}{\sigma_{D}} .
$$

Using (E.17), as $\omega_{t}$ approaches zero we obtain

$$
\begin{aligned}
& \lim _{\omega_{t} \rightarrow 0}\left(1+\frac{1-\omega_{t}}{\omega_{t}} \tau y_{t}\left(1+\frac{\varphi}{y_{t}} \frac{d y_{t}}{d \varphi}\right)\right)=1+\tau \lim _{\omega_{t} \rightarrow 0} x \times \lim _{\omega_{t} \rightarrow 0}\left(1+\frac{\varphi}{y_{t}} \frac{d y_{t}}{d \varphi}\right) \\
& =\tau \lim _{\omega_{t} \rightarrow 0} x \times\left(1-\frac{\frac{\varphi}{\sigma_{D}}}{\sigma_{D} \lim _{\omega_{t} \rightarrow 0} x_{t}}\right) \\
& =\tau\left(\frac{\eta-\sigma_{D}-2 \frac{\varphi}{\sigma_{D}}}{\sigma_{D}}\right) \\
& <0 \text {, }
\end{aligned}
$$

where we used $(21)$ to derive the last inequality. Combining $($ E.18) with (E.15) implies that, for small $\omega_{t}, \frac{d \kappa\left(y^{+}\right)}{d \varphi}>0$.

Proof of Proposition 5. The proof essentially repeats the steps from the one-risky asset case, so we provide only a sketch, focusing on the elements that differ. We define

$$
\vec{\varphi}=\left[\begin{array}{l}
\varphi \\
0
\end{array}\right], \vec{\eta}=\left[\begin{array}{l}
\eta \\
0
\end{array}\right]
$$

We consider first an equilibrium with $y_{t}>0$. Investor $R$ 's and $I$ 's optimal portfolios are given by

$$
\begin{aligned}
& \vec{w}_{t}^{R}=\left(\sigma_{t} \sigma_{t}^{\prime}\right)^{-1}\left(\vec{\mu}_{t}-r_{t} 1_{2 \times 1}+\vec{\varphi}\right), \\
& \vec{w}_{t}^{I}=\left(\sigma_{t} \sigma_{t}^{\prime}\right)^{-1}\left(\vec{\mu}_{t}-r_{t} 1_{2 \times 1}+\sigma_{1, t} \vec{\eta}+\tau y_{t} \vec{\varphi}\right) .
\end{aligned}
$$

Using E.19) inside (38) yields

$$
\begin{aligned}
\left(\sigma_{t} \sigma_{t}^{\prime}\right) \vec{m}_{t}= & \widehat{\omega}_{t}\left[\omega_{t}\left(\vec{\mu}_{t}-r 1_{N}+\vec{\varphi}\right)+\left(1-\omega_{t}\right)\left(\vec{\mu}_{t}-r 1_{N}+\sigma_{1} \vec{\eta}+\tau y_{t} \vec{\varphi}\right)\right] \\
& +\left(1-\widehat{\omega}_{t}\right)\left(\sigma_{t} \sigma_{t}^{\prime}\right)\left[\begin{array}{c}
0 \\
\frac{\mu_{2, t}-r}{\sigma_{2, t}^{2}}
\end{array}\right] .
\end{aligned}
$$

Next we use the row selection vector $[0,1]$ to pre-multiply both sides of $($ E.21). Noting that $[0,1] \vec{\varphi}=[0,1] \vec{\eta}=0$, and also

$$
\left(\sigma_{t} \sigma_{t}^{\prime}\right)\left[\begin{array}{c}
0 \\
\frac{\mu_{2, t}-r}{\sigma_{2, t}^{2}}
\end{array}\right]=\left[\begin{array}{c}
b_{t}\left(\mu_{2, t}-r\right) \\
\mu_{2, t}-r
\end{array}\right],
$$


leads to (41). We next note that

$$
\begin{aligned}
{\left[1,-b_{t}\right] \sigma_{t} \sigma_{t}^{\prime}\left[\begin{array}{l}
m_{1, t} \\
m_{2, t}
\end{array}\right] } & =\left[\sigma_{1, t}, 0\right]\left[\begin{array}{cc}
\sigma_{1, t} & 0 \\
b_{t} \sigma_{2, t} & \sigma_{2, t}
\end{array}\right]\left[\begin{array}{l}
m_{1, t} \\
m_{2, t}
\end{array}\right] \\
& =\sigma_{1, t}^{2} m_{1, t}
\end{aligned}
$$

Pre-multiplying both sides of (E.21) with the row vector $\left[1,-b_{t}\right]$, using (E.22), (E.23), and the definition of $\kappa_{1, t}$, and re-arranging yields

$$
\kappa_{1, t}=\widetilde{m}_{1, t} \sigma_{1, t}-\left(1-\omega_{t}\right) \eta-\frac{\varphi}{\sigma_{1, t}}\left(\omega_{t}+\left(1-\omega_{t}\right) \tau y_{t}\right)
$$

Using the definition of $\kappa_{1, t}$ inside (E.19) gives

$$
\begin{aligned}
& w_{1, t}^{R}=\frac{\kappa_{1, t}}{\sigma_{1, t}}+\frac{\varphi}{\sigma_{1, t}^{2}} \\
& w_{1, t}^{I}=\frac{\kappa_{1, t}+\eta}{\sigma_{1, t}}+\frac{\tau y_{t} \varphi}{\sigma_{1, t}^{2}},
\end{aligned}
$$

where we used the notation $w_{t}^{1, i}, i \in\{R, I\}$, to denote the first element of $w_{t}^{i}$.

Using the market clearing condition $y_{t}=-\frac{\omega_{t}^{R} w_{1, t}^{R}}{\omega_{t}^{I} w_{1, t}^{I}}=-\frac{\omega_{t} w_{1, t}^{R}}{\left(1-\omega_{t}\right) w_{1, t}^{I}}$ leads to 39 .

If agent $R$ chooses not to short then the market clearing condition becomes

$$
\widehat{\omega}_{t}\left(1-\omega_{t}\right) w_{t}^{I}+\left(1-\widehat{\omega}_{t}\right)\left[\begin{array}{c}
0 \\
\widehat{w}_{2, t}
\end{array}\right]=\vec{m}_{t} .
$$

Substituting (E.20), pre-mutiplying by $\left(\sigma_{t} \sigma_{t}^{\prime}\right)$ gives

$$
\left(\sigma_{t} \sigma_{t}^{\prime}\right) \vec{m}_{t}=\widehat{\omega}_{t}\left(1-\omega_{t}\right)\left(\vec{\mu}_{t}-r 1_{N}+\sigma_{1} \vec{\eta}\right)+\left(1-\widehat{\omega}_{t}\right)\left(\sigma_{t} \sigma_{t}^{\prime}\right)\left[\begin{array}{c}
0 \\
\frac{\mu_{2, t}-r}{\sigma_{2, t}^{2}}
\end{array}\right] .
$$

Premultiplying $(\mathrm{E} .28)$ by the row $\left[1,-b_{t}\right]$ and using $(\mathrm{E} .22)$ and $(\mathrm{E} .23)$ gives

$$
\sigma_{1, t}^{2} \widetilde{m}_{1, t}=\left(1-\omega_{t}\right) \sigma_{1, t}\left(\kappa_{1, t}+\eta\right)
$$

and therefore

$$
\kappa_{1, t}=\sigma_{1, t} \frac{\widetilde{m}_{1, t}}{1-\omega_{t}}-\eta
$$

Finally, when both agents hold positive portfolios, the optimal portfolios are $\vec{w}_{t}^{R}=\left(\sigma_{t} \sigma_{t}^{\prime}\right)^{-1}\left(\vec{\mu}_{t}-r_{t} 1_{2 \times 1}\right)$, $\vec{w}_{t}^{I}=\left(\sigma_{t} \sigma_{t}^{\prime}\right)^{-1}\left(\vec{\mu}_{t}-r_{t} 1_{2 \times 1}+\sigma_{1, t} \vec{\eta}\right)$. Repeating the arguments in equations E.19)-E.24, we obtain $\kappa_{1, t}=\widetilde{m}_{1, t} \sigma_{1, t}-\left(1-\omega_{t}\right) \eta$.

Proof of Proposition 6. The proof of this Proposition is contained in Appendix C.

Proof of Proposition 7. Since this proof is essentially identical to the proof of Proposition 2, we only provide a sketch. Combining (14) with $\sum_{i} \omega_{t}^{i} w_{t}^{i}=1$ implies that in 
any equilibrium with $w_{t}^{R}<0$ and $w_{t}^{I}>0$ the Sharpe ratio is

$$
\kappa_{t}+\left(1-\omega_{t}\right) \eta+\omega_{t} \frac{1}{\sigma_{D}} f_{t}+\left(1-\omega_{t}\right) \tau y_{t} \frac{1}{\sigma_{D}} f_{t}=\sigma_{D}
$$

Re-arranging (E.30) and using $f_{t}=l\left(y_{t}\right)$ gives (D.3). Substituting (D.3) back into the investors' optimal portfolios 14 and the fact that $y_{t}=-\frac{\omega_{t} w_{t}^{R}}{\left(1-\omega_{t}\right) w_{t}^{I}}$ leads to (D.2).

We next study the roots of $(\mathrm{D} .2)$. Let $Z(y) \equiv \frac{\eta-\frac{\sigma_{D}}{1-\omega_{t}}-\frac{1}{\sigma_{D}} z(y)}{\eta+\frac{\sigma_{D}}{\omega_{t}}-\frac{1}{\sigma_{D}} z(y)}$, so that equation $(\mathrm{D} .2)$ can be expressed as $y=Z(y)$. The assumption of the proposition is that there exists at least one $y$ such that $y=Z(y)$. Let $\bar{y}^{*}$ be the largest root of $(\mathrm{D} .2)$ that satisfies $\eta-\frac{\sigma_{D}}{1-\omega_{t}}-\frac{1}{\sigma_{D}} z(y)>0$. We consider two cases: i) $\eta-\frac{\sigma_{D}}{1-\omega_{t}}-\frac{1}{\sigma_{D}} z(y)>0$ for all $y \in\left[\bar{y}^{*}, 1\right]$ and ii) $\eta-\frac{\sigma_{D}}{1-\omega_{t}}-\frac{1}{\sigma_{D}} z(\bar{y})=0$ for some $\bar{y} \in\left[\bar{y}^{*}, 1\right]$. In case i) it must be that $Z^{\prime}\left(\bar{y}^{*}\right) \leq 1$, since $\bar{y}^{*}=Z\left(\bar{y}^{*}\right)$ and $1>Z(1)$. In case ii) it must also be that $Z^{\prime}\left(\bar{y}^{*}\right) \leq 1$ since $\bar{y}>Z(\bar{y})=0$.

Furthermore, by continuity values of $y$ lower than $\bar{y}^{*}$ exist such that $\eta-\frac{\sigma_{D}}{1-\omega_{t}}-\frac{1}{\sigma_{D}} z(y)=0$; let $y$ be the highest such value and note $0<y<\bar{y}^{*}$. Since the numerator of $Z$ is positive on $\left(\underline{y}, \bar{y}^{*}\right)$, so is the denominator. Moreover, we have $Z(\underline{y})=0<\underline{y}$. A solution $Z(y)=y$ therefore exists in $\left(\underline{y}, \bar{y}^{*}\right)$, and it defines a second equilibrium with strictly positive short interest.

Finally, to confirm that a no-shorting equilibrium is also an equilibrium, $\eta-\frac{\sigma_{D}}{1-\omega_{t}}-$ $\frac{1}{\sigma_{D}} z(0)<0$ is equivalent to $\eta-\frac{\sigma_{D}}{1-\omega_{t}}-\frac{1}{\sigma_{D}} l(0)<0$. If the Sharpe ratio is given by $\kappa_{t}=$ $\frac{\sigma_{D}}{1-\omega_{t}}-\eta<0$, the assumption $\eta-\frac{\sigma_{D}}{1-\omega_{t}}-\frac{1}{\sigma_{D}} l(0)<0$ implies $\kappa_{t}+\frac{1}{\sigma_{D}} l(0)>0$. Accordingly, investor $R$ does not wish to short when the fee is $f_{t}=l(0)$ and the lending market clears with $y=0$ at the lending fee $l(0)$. Moreover, $w_{t}^{I}=\frac{\kappa_{t}+\eta}{\sigma_{D}}=\frac{1}{1-\omega_{t}}$. Therefore $\omega_{t} \times 0+\left(1-\omega_{t}\right) \times w_{t}^{I}=1$ and the stock market clears. 


\section{F Additional Data Discussion}

\section{F.1 Methodology}

\section{F.1.1 Measuring ticker discussion on WallstreetBets}

Our measure of ticker mentions on WallstreetBets is constructed as follows. We use the PushshiftAPI to collect all submissions posted on WallstreetBets subreddit from January 1, 2020 through February 7, 2021 (Baumgartner et al., 2020). For each submission, we observe the title text, the body of the submission, the author of the submission, and the time of the submission.

In order to identify which tickers are discussed in the submission, we take advantage of the fact that users often tag tickers with a leading $\$$ (i.e. \$TSLA or \$AAPL). This practice is entirely voluntary and is therefore insufficient for identifying all mentions of a ticker. We use regular expressions to identify all words tagged in this way and match those words to CRSP tickers that were traded on the NYSE, AMEX, and NASDAQ exchanges in 2020. This gives us a set $S$ of roughly 4,000 tickers that are mentioned on WSB between January 2020 and February 2021.

We then identify all cases in which these tickers are mentioned in submissions, irrespective of whether they are prefixed with a dollar sign. To address the possibility of falsely identifying tickers, we require that, if the ticker is a common word in the written English language, it must be prefaced by a dollar sign. For example, AT\&T's ticker T is also a common word in written English, and thus we require that the text "\$T" appear in a submission for it to be considered as mentioned AT\&T. We consider a ticker as being mentioned in a submission if it appears in either the title or the body of the submission. We identify common word-stems based on the Google Trillion Word Corpus (Michel et al., 2011). In a robustness check, we account for the downward bias this restriction introduces by scaling common-word tickers by an in-sample estimated adjustment factor. This adjustment leaves the relative ranking of ticker mentions largely unchanged. We estimate the adjustment factor by comparing the frequency of tagged ticker mentions versus untagged ticker mentions for the set of tickers which do not commonly appear in written English.

Revised submissions and comments. Authors of Reddit comments have the ability to edit their comments even after the comment has been posted. The PushshiftAPI records the comment text as of a certain day, and does not update to reflect potential revised comments. The same constraint applies to the content body of submissions. Titles of submissions cannot be revised and thus do not have this measurement problem.

Missed tickers Tickers that, for whatever reason, are never tagged with a leading dollar sign will be omitted from our dataset. Similarly, we under-count the occurrences of tickers that are common words, owing to requiring they appear with a leading "\$" We attempt to correct for this by scaling the observed counts for common word tickers. For AAPL and GME, which are not common word tickers, the ticker appears with the leading "\$" roughly $20 \%$ of the time. We can thus simply multiply our observed frequencies by a factor of five to adjust for the more stringent matching procedure. As can be seen in Figures 14a and 


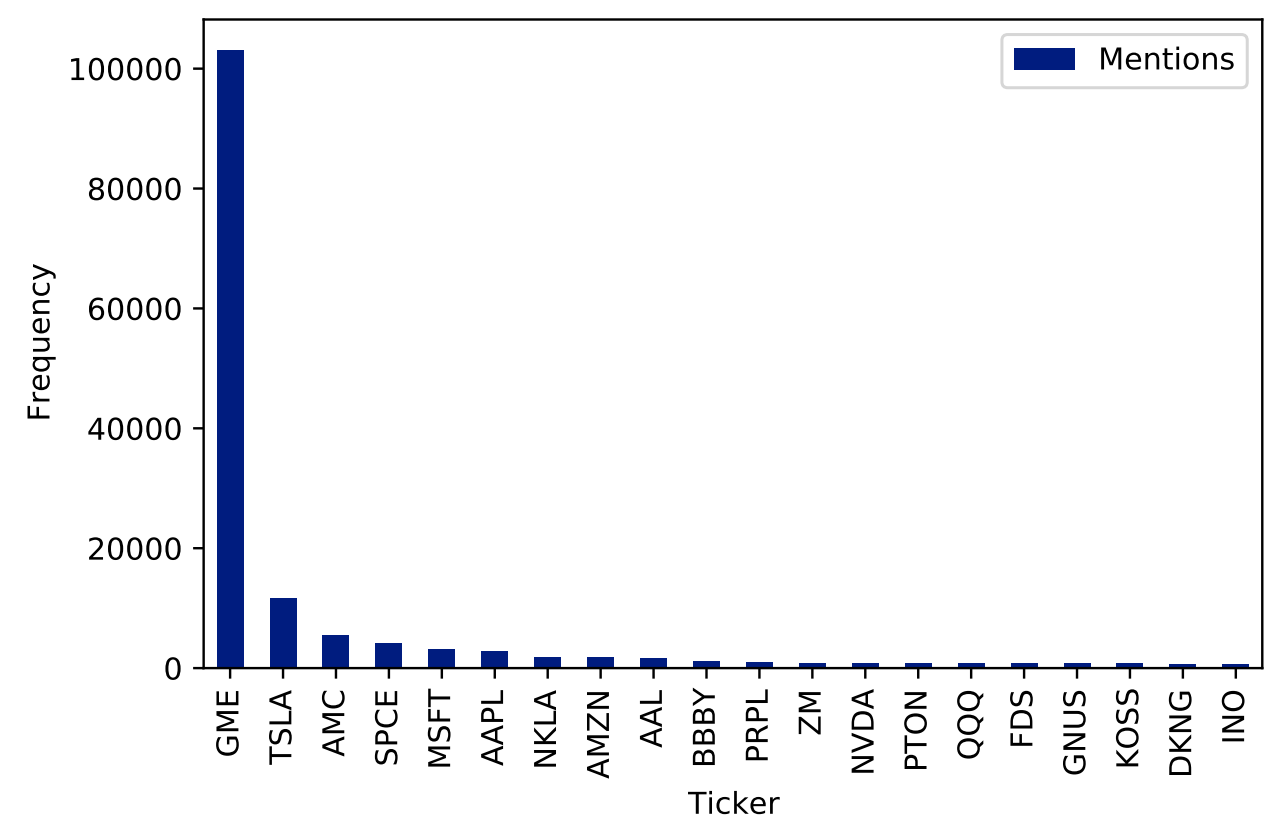

(a) Submissions mentioning each Ticker

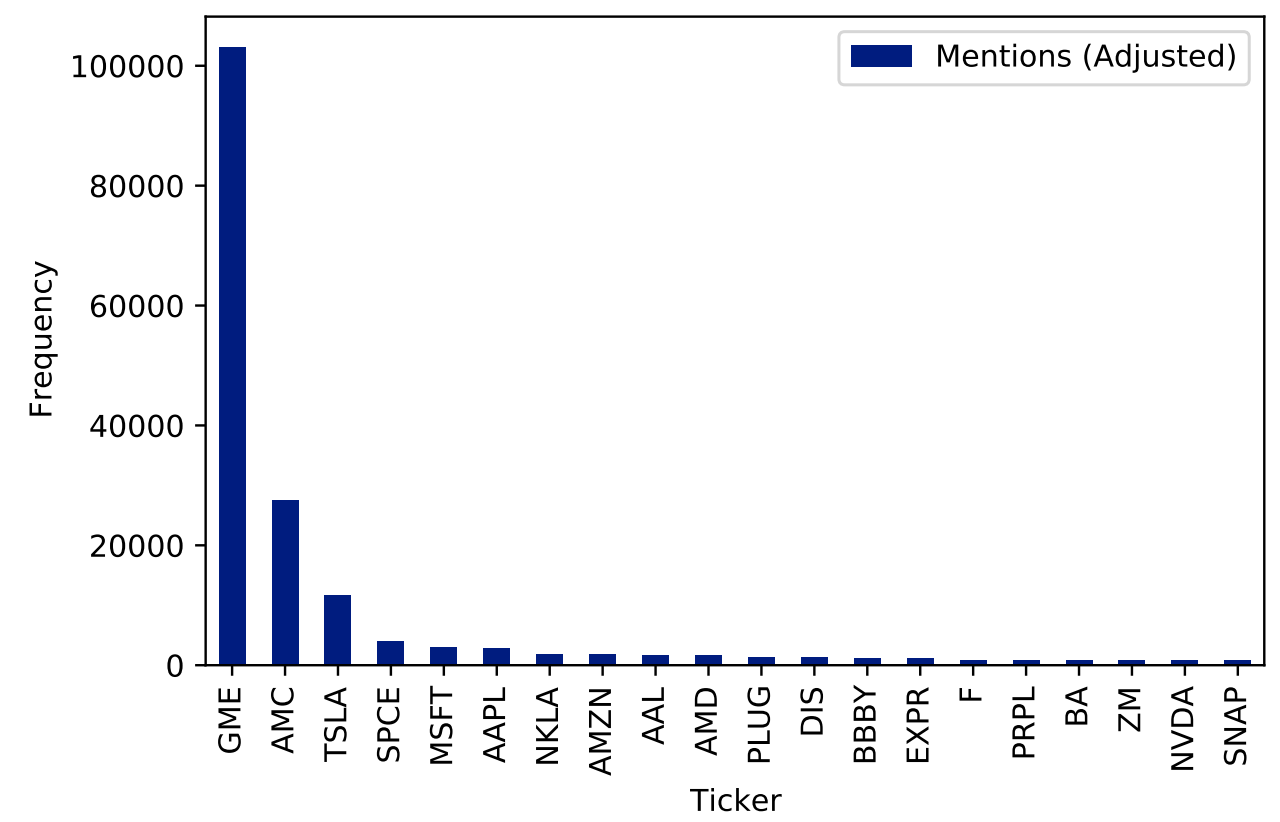

(b) Submissions mentioning each Ticker, adjusted for word-ticker overlap

Figure 14: Popular Tickers on WallstreetBets (January 1, 2020 - February 7, 2021).

$14 \mathrm{~b}$, the adjustment does not have a significant impact on the relative popularity of the top tickers.

In some cases, users may choose to refer to the company by its name, rather than by its ticker. We do not attempt to identify mentions of companies by name. 


\section{F.2 Measuring retail trading}

We adopt the methodology of Boehmer et al. (2020) to identify retail trades in the TAQ data. We briefly summarize the methodology here and refer readers to the paper for details.

The intuition behind the methodology is the knowledge that retail trades are often executed by wholesalers or via broker internalization, rather than on the major trading exchanges. These trades appear in the TAQ consolidated tape data under the exchange code "D." These trades are given a small price improvement on the order of tenths of a penny as a means to induce brokers to route orders to the wholesaler. Similarly, brokers which internalize retail trades offer a subpenny price improvement in order to comply with Regulation 606T. Importantly, institutional trades are rarely, if ever, internalized or directed to wholesalers and their trades are usually in round penny prices, with the notable exception of midpoint trades.

The methodology of Boehmer et al. (2020) uses these institutional details to identify retail trades in the TAQ consolidated tape data. Trades flagged with exchange code "D" and with a subpenny amount in the set $(0,0.40) \cup(0.60,1.00)$ are identified as retail trades. Splitting these trades further, retail trades with subpenny amounts between zero- and fortyhundredths of a penny are labeled as "sell orders," whereas subpenny amounts between sixty- and one hundred-hundredths are considered "buy orders." The midpoint trades are excluded to avoid mis-classifying institutional trades executed at midpoints as retail trades.

\section{F.2.1 Challenges}

Derivatives The TAQ data only contains trades of equities. Options offer another way to benefit for investors to benefit from increases in the price of stock. As an added advantage for retail investors, options offer embedded leverage greater than what might otherwise be available through their broker. The Boehmer et al. (2020) methodology relies on institutional details to identify off-exchange retail trades, and thus cannot reliably identify replication trades by market makers.

\section{F.3 Betting against the shorts portfolio}

As is standard in the literature, we restrict attention to common shares of COMPUSTAT firms which trade on the NYSE, AMEX, and NASDAQ exchanges. We further exclude companies for whom no share class has a price exceeding $\$ 1$. The strategy equally weights each firm in the top decile, shorts the market index, and reconstitutes 8 trading days following the disclosure date, which is the first opportunity following the public dissemination of the short interest data. 


\section{G Additional Table and Figures}

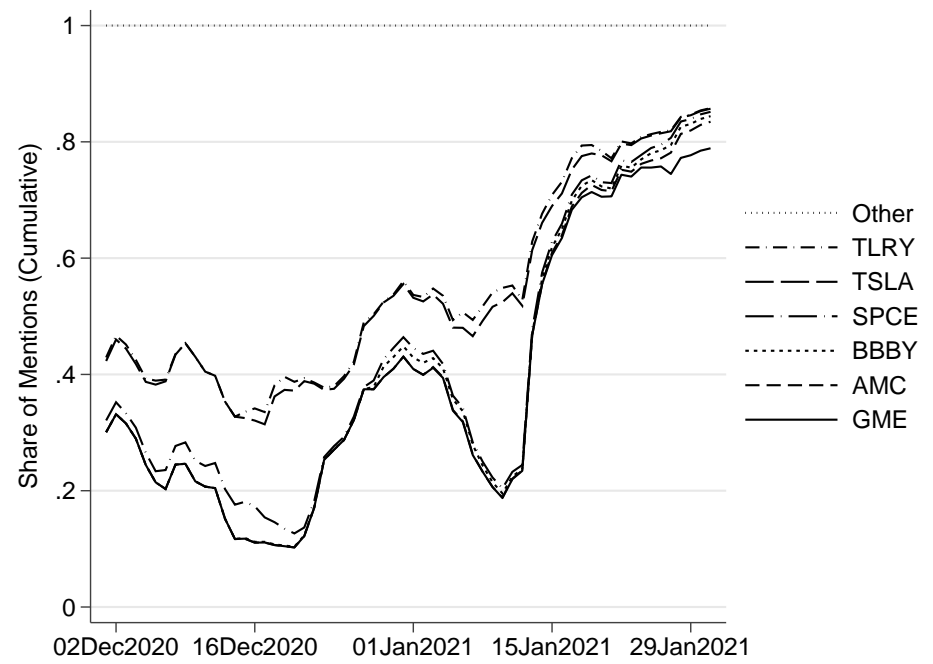

Figure 15: Cumulative fraction of discussion (December 1, 2020 - January 31, 2021). Relative shares for ticker $s$ are computed as $m_{s t}=\frac{\text { Mentions }_{s t}}{\sum_{s^{\prime} \in S} \text { Mentions }_{s^{\prime} t}}$. "Other" consists of all other tickers mentioned on WSB. 


\begin{tabular}{|c|c|c|c|}
\hline & Highly Shorted Stocks & Excl. Popular Reddit Stocks & Excl. Small Stocks \\
\hline \multicolumn{4}{|c|}{ Panel A: November 2020} \\
\hline \multirow[t]{2}{*}{$r^{E W}$} & 0.163 & 0.160 & 0.227 \\
\hline & $(4.127)$ & $(4.050)$ & $(5.194)$ \\
\hline \multirow{2}{*}{$r^{V W}$} & 0.094 & 0.092 & 0.133 \\
\hline & $(3.062)$ & $(3.029)$ & $(3.455)$ \\
\hline \multirow{2}{*}{$r_{\mathrm{FF} 3}^{E W}$} & 0.084 & 0.081 & 0.160 \\
\hline & $(3.452)$ & $(3.327)$ & $(4.371)$ \\
\hline \multirow[t]{2}{*}{$r_{\mathrm{FF} 3}^{V W}$} & 0.045 & 0.043 & 0.083 \\
\hline & $(1.769)$ & $(1.706)$ & $(2.431)$ \\
\hline \multicolumn{4}{|c|}{ Panel B: December 2020 } \\
\hline \multirow[t]{2}{*}{$r^{E W}$} & 0.055 & 0.058 & 0.019 \\
\hline & $(1.385)$ & $(1.477)$ & $(0.437)$ \\
\hline \multirow{2}{*}{$r^{V W}$} & 0.033 & 0.036 & 0.021 \\
\hline & $(1.088)$ & $(1.191)$ & $(0.540)$ \\
\hline \multirow{2}{*}{$r_{\mathrm{FF} 3}^{E W}$} & 0.012 & 0.016 & -0.002 \\
\hline & $(0.515)$ & $(0.665)$ & $(-0.056)$ \\
\hline \multirow{2}{*}{$r_{\mathrm{FF} 3}^{V W}$} & 0.012 & 0.014 & 0.008 \\
\hline & $(0.466)$ & $(0.576)$ & $(0.244)$ \\
\hline \multicolumn{4}{|c|}{ Panel C: January 2021} \\
\hline \multirow[t]{2}{*}{$r^{E W}$} & 0.271 & 0.232 & 0.156 \\
\hline & $(6.835)$ & $(5.865)$ & $(3.576)$ \\
\hline \multirow[t]{2}{*}{$r^{V W}$} & 0.194 & 0.161 & 0.183 \\
\hline & $(6.341)$ & $(5.293)$ & $(4.764)$ \\
\hline \multirow[t]{2}{*}{$r_{\mathrm{FF} 3}^{E W}$} & 0.208 & 0.169 & 0.121 \\
\hline & $(8.560)$ & $(6.978)$ & $(3.296)$ \\
\hline \multirow{2}{*}{$r_{\mathrm{FF} 3}^{V W}$} & 0.171 & 0.136 & 0.165 \\
\hline & $(6.709)$ & $(5.452)$ & $(4.816)$ \\
\hline
\end{tabular}

Table 1: Portfolio returns (November 2020-January 2021). Test of whether the monthly return to the strategy of betting against the shorts is "abnormal" in November 2020 (Panel A), December 2020 (Panel B), and January 2021 (Panel C). The table reports the coefficient and the $t$-statistic of the month dummy variable that takes the value of one for the month listed in the title of the panel and zero otherwise from the regression:

$$
r_{\text {Betting against the shorts }}=\text { const. }+ \text { month dummy }+\beta^{\prime} F_{t}+\varepsilon_{t} .
$$

The first two rows of each panel do not control for any factor exposures and refer to equalweighted $(E W)$ and value-weighted $(V W)$ returns, respectively. The last two rows of each panel control for Fama-French 3-factor exposures. 

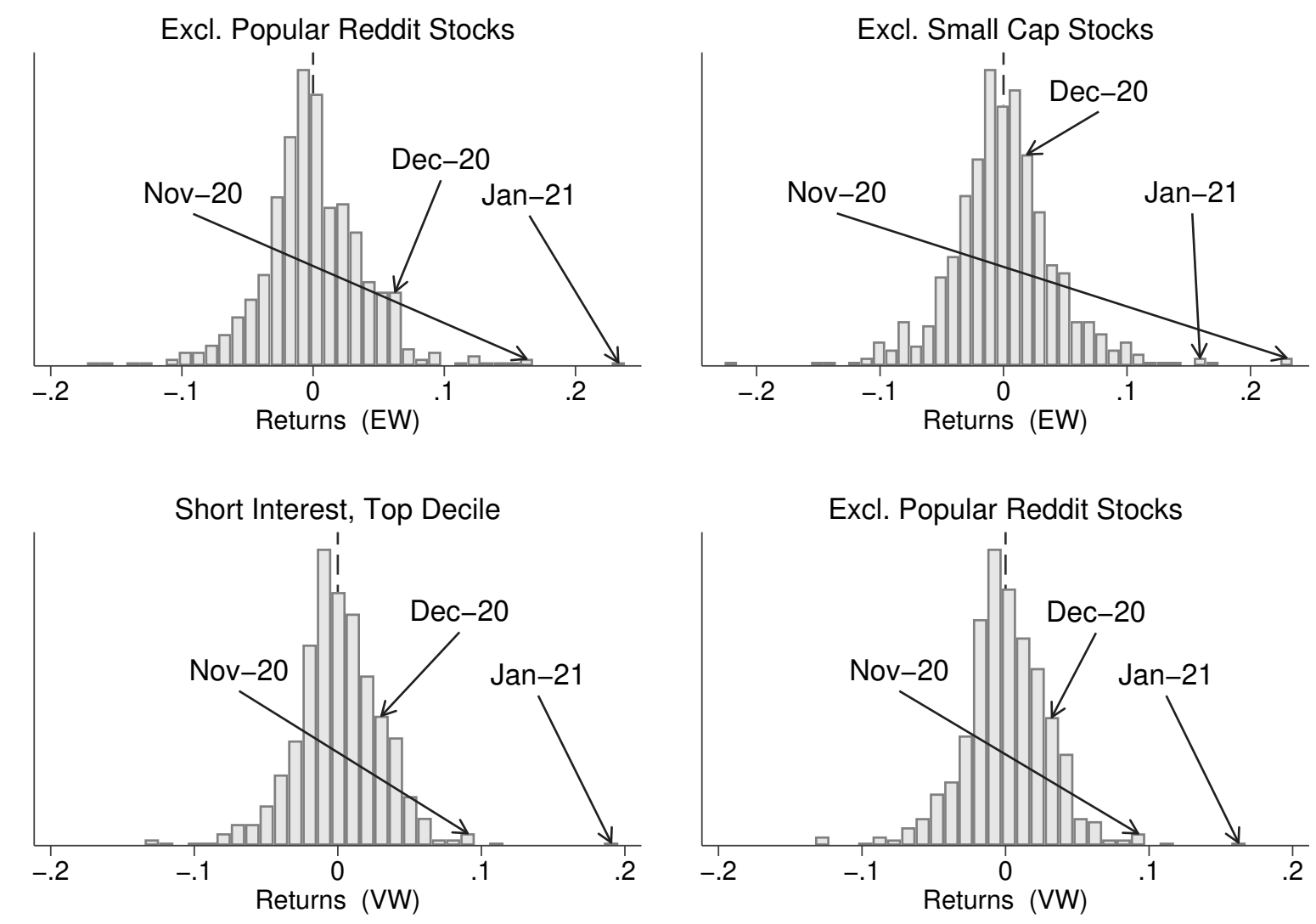

Figure 16: Monthly returns (1973-2021). Histograms show monthly returns to a trading strategy long stocks in the top decile of short interest and short the market index. The topleft plot depicts equal-weighted returns, excluding the six most-popular stocks discussed on Reddit (AMC, BBBY, GME, SPCE, TLRY, and TSLA). The top-right plot depicts equalweighted returns, further excluding small market capitalization stocks. The bottom-left plot depicts value-weighted returns. The bottom-right plot depicts value-weighted returns, excluding popular stocks discussed on Reddit. The arrows indicate the portfolio returns in the months of November and December 2020 and January 2021. 

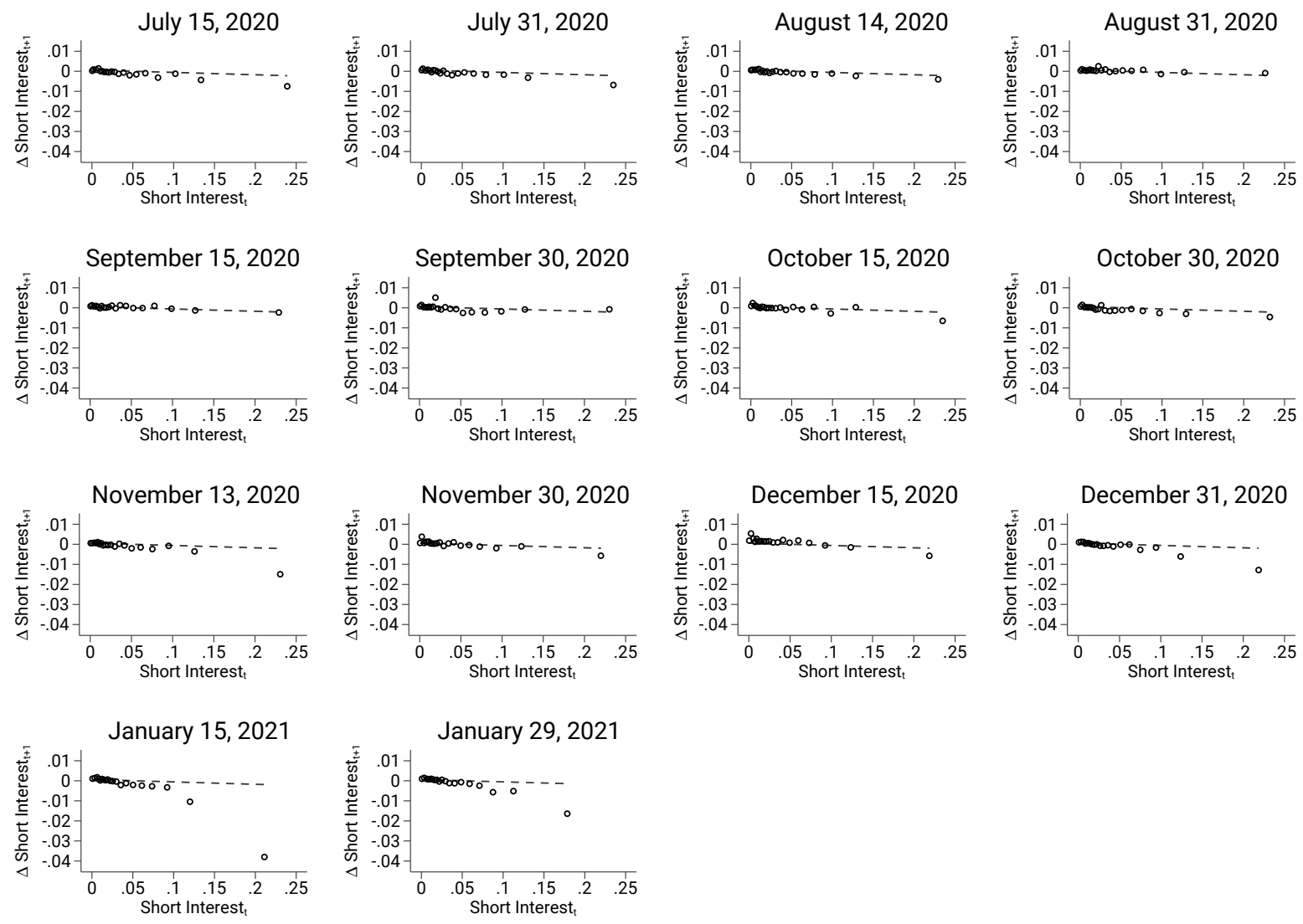

Figure 17: Binned scatterplots of changes in short interest by decile of short interest (July 2020-January 2021). The figure repeats the exercise of Figure 5 for the second half of 2020. Each panel is a binned scatterplot of short interest as of an SEC disclosure date against changes in short interest as of the subsequent disclosure date. The average percentage point change for each bin is plotted on the y-axis. The average percentage point short interest for each bin is plotted on the x-axis. The dashed line indicates the predicted change in short interest based on $\mathrm{AR}(1)$ models for each decile of short interest, fitted on historical shorting data. 


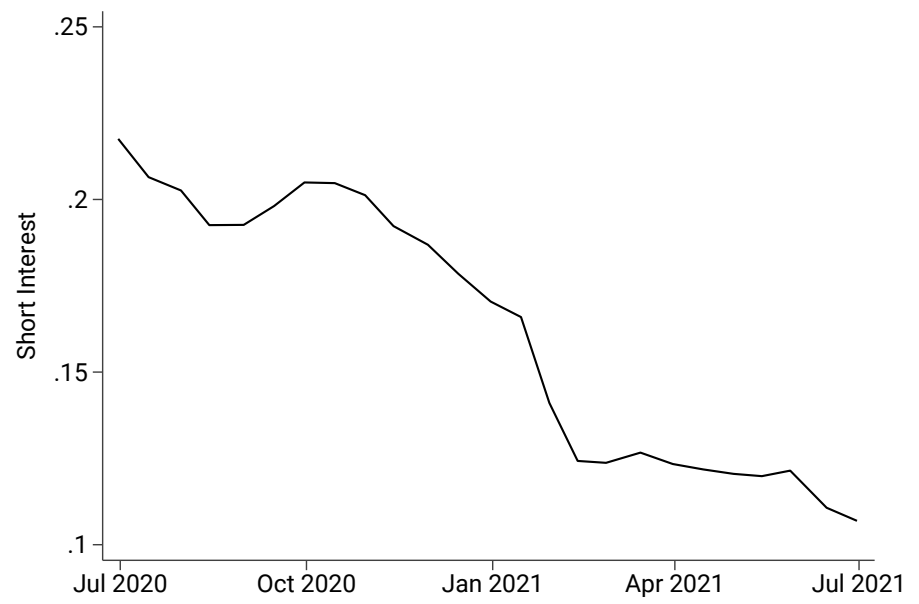

Figure 18: Aggregate short interest (July 2020-June 2021). The figure plots value-weighted short interest for highly shorted stocks as of October 31, 2020. Highly shorted stocks are defined as the stocks in the top decile of the Russell 3000, ranked by short interest. The identities of these stocks is fixed and their short interest is plotted over the preceding four and subsequent eight months. 